Theta functions and non-linear equations

This article has been downloaded from IOPscience. Please scroll down to see the full text article.

1981 Russ. Math. Surv. 3611

(http://iopscience.iop.org/0036-0279/36/2/R02)

View the table of contents for this issue, or go to the journal homepage for more

Download details:

IP Address: 147.122.45.69

The article was downloaded on 31/01/2013 at 15:01

Please note that terms and conditions apply. 


\title{
Theta functions and non-linear equations
}

\author{
B.A. Dubrovin
}

CONTENTS

Introduction $\quad 11$

Chapter 1. Theta functions. General information 14

$\S 1$. Definition of theta functions and their simplest properties 14

$\begin{array}{ll}\S 2 \text {. The ta functions of a single variable } & 17\end{array}$

§3. On Abelian tori 18

\$4. Addition theorems for theta functions 22

Chapter II. Theta functions of Riemann surfaces. The Jacobi inversion problem 24

$\S 1$. Periods of Abelian differentials on Riemann surfaces. Jacobi varieties 24

\$2. Abel's theorem 30

$\S 3$. Some remarks on divisors on a Riemann surface 31

\$4. The Jacobi inversion problem. Examples 34

Chapter IIl. The Baker-Akhiezer function. Applications to non-linear equations 44

$\S 1$. The Baker-Akhiezer one-point function. The Kadomtsev-Petviashvili equation and equations associated with it

$\S 2$. The Baker-Akhiezer two-point function. The Schrödinger equation in a magnetic field

Chapter IV. Effectivization of the formulae for the solution of KdV and KP equations. Recovery of a Riemann surface from its Jacobi variety. The $\begin{array}{ll}\text { problem of Riemann and the conjecture of Novikov } & 58\end{array}$

$\S 1$. The KdV equation. Genus $g=1$ or $2 \quad 58$

\$2. The KP equation. Genus 2 and $3 \quad 62$

$\S 3$. The KP equation. Genus $g \geqslant 2$. Canonical equations of Riemann surfaces. 65

$\S 4$. The problem of Riemann on relations between the periods of holomorphic differentials on a Riemann surface and the conjecture of Novikov $\quad 70$

Chapter V. Examples of Hamiltonian systems that are integrable in terms of $\begin{array}{ll}\text { two-dimensional theta functions } & 72\end{array}$

$\begin{array}{ll}\S 1 . & 72\end{array}$

$\S 2$. The problem of Sophie Kovalevskaya 75

$\S 3$. The problems of Neumann and Jacobi. The general Garnier system 76

\$4. Movement of a solid in an ideal fluid. Integration of the Clebsch case. A multi-dimensional solid

Appendix. I.M. Krichever. The periodic non-Abelian Toda chain and its twodimensional generalization 


\section{Introduction}

In recent years the development of methods for the exact solution of nonlinear equations of mathematical physics (the inverse scattering method; see [13] and the literature cited there) has involved several function-theoretical constructions related to Riemann surfaces. This refers first of all to the theory of multidemensional theta functions in terms of which one can express the so-called "rank 1 solutions" of a wide class of non-linear equations (the theory of solutions of higher rank is connected with holomorphic vector bundles over Riemann surfaces and is presented in the survey [14]; a complete bibliography is given there). In the present survey we present the basic principles of the application of theta functions to the integration of equations, together with important examples of this application.

We recall briefly the history of this question. In 1974 in a series of papers Novikov, the present author, Matveev and Its ([20], [53], [54], [60], [17]), and Lax ([55]) introduced and studied the class of "finite-zone" periodic and quasi-periodic potentials of the Schrödinger operator (SturmLiouville, Hill). On the basis of this class a program for the construction of a wide class of solutions of the Korteweg-de Vries (KdV) equation was proposed and realized. Some results of these studies were also obtained by McKean and van Moerbeke in 1975 [56]. As was later proved rigorously (Marchenko and Ostrovskii [57]), the set of periodic finite-zone potentials is dense in the space of periodic functions with given period. In these articles a connection was established between the spectral theory of operators with periodic coefficients and algebraic geometry, the theory of finite-dimensional completely integrable Hamiltonian systems and the theory of non-linear equations of $\mathrm{KdV}$ type. A generalization of this theory to spatially twodimensional (" $2+1 " ; x, y, t)$ systems, among which there is the important two-dimensional analogue of the KdV equation - the Kadomtsev-Petviashvili equation (KP) - was realized by Krichever [59], [15], [16]. Krichever's approach also gives a methodologically very convenient and lucid presentation of an algebro-geometric procedure of constructing the above-mentioned finite-zone solutions of the $\mathrm{KdV}$ equation and its numerous analogues. In the case of $(2+1)$-systems this method reveals new important connections with algebraic geometry, which are used essentially in the statement of the problem of Chapter IV of this paper.

As Novikov and Natanzon have pointed out to the author, the determination of conditions for the selection of real solutions in various problems of this kind turns out to be a non-trivial and as yet unsolved problem, except for individual cases literally analogous to the $\mathrm{KdV}$ equation, which do not, however, include the sine-Gordon equation or the non-linear Schrödinger equation with repulsing interaction, nor all the $(2+1)$-systems. An essential advance was made by Cherednik in [49] and developed in [14]. The results of [49] hardly pretend to completeness and are not effective even in the simplest cases. 
The first two chapters of this survey are devoted to the most important results of the theory of theta functions, their connection with Riemann surfaces and Abelian varieties. The material of these chapters basically goes back to the classical works of Abel, Jacobi, and Riemann (see the books [1] - [8]; for a modern account a detailed bibliography can be found in [4]). In the third chapter we explain Krichever's method (see [15], [16]) for constructing exact solutions of non-linear equations (in \$2 we also use results of [21]). This method allows us to express the solutions thus constructed in terms of theta functions. The basic instrument for constructing such solutions is the so-called Baker-Akhiezer functions, that is, meromorphic functions on a Riemann surface with an essential singularity of a given form (see [18], [19]; apparently, the first functions of this type were considered by Clebsch and Gordan). It is essential to note that in the applications to non-linear equations it is not arbitrary theta functions that arise, but only theta functions of Riemann surfaces. Novikov has conjectured that the identities on a theta function (or on the Riemann matrix defining it), obtained after making an elementary substitution of it in the KadomtsevPetviashvili (KP) equation, precisely distinguish the theta functions of Riemann surfaces from all other theta functions. A partial realization of this programthe derivation of a system of identities connecting the parameters of a theta function of Riemann surfaces (without proving the completeness of this system) - was obtaine $d^{(1)}$ by the author in [23]); the corresponding results are presented in Chapter 4. Incidentally we also solve Novikov's problem on the effectivization of formulae for the solution of non-linear equations in the case of small genera, where restrictions on the theta functions do not yet arise. Moreover, the use of the KP equation allows us to give an explicit construction for the recovery of an algebraic curve from its Jacobian (that is, to give a new proof to the classical Torelli theorem, which asserts the uniqueness of this correspondence; see [4]), and this construction does not require a knowledge of the solutions of the transcendental equation $\theta(z)=0$. Very recently the author has succeeded in proving Novikov's conjecture in a weaker version: the relations on a theta function that follow from the KP equation distinguish the variety of theta functions of Riemann surfaces in the space of all theta functions up to possibly superfluous irreducible components. The idea of the proof is also given in Chapter 4. Thus, the use of the KP equation allows us effectively to solve the classical problem of Riemann on the relations between the periods of holomorphic differentials on Riemann surfaces. Finally, in the last Chapter we list the dynamical

${ }^{(1)}$ The statements of some results of [23] were published (and used) in [37]. We also mention that after [23] was published, the author was shown a preprint by Hirota [24] ; the methods of this preprint intersect with some technical arguments of [23]. Hirota solves the problem of constructing exact solutions of non-linear equations of KdV type by means of the theory of theta functions. The account in [24] does not give explicit formulae. 
systems that are integrable in terms of theta functions of genus 2. Except for equations of the theory of two-zone potentials, which are interpreted in [20] and [17], and also multidimensional Euler equations, all these systems are classical, although the structure of their solutions is not well known. The invariant varieties of these systems are Abelian varieties of genus 2 . This allows us to obtain explicit formulae of various types for the universal bundle of Abelian varieties of genus 2 (the first such formulae were obtained in [35]). As an application we give the work of Krichever on the integration of the non Abelian Toda chain by algebraic-geometric methods.

The author thanks S.P. Novikov and I.M. Krichever for their interest in this work and for a number of useful discussions. In the process of writing Chapter 4 the author consulted A.N. Tyurin, to whom he expresses his deep appreciation.

\section{CHAPTER I}

THETA FUNCTIONS, GENERAL INFORMATION

$\S 1$. Definition of theta functions and their simplest properties

Definition 1.1.1. A symmetric $(g \times g)$-matrix $B=\left(B_{j k}\right)$ with negative definite real part $\operatorname{Re} B=\left(\operatorname{Re} B_{j k}\right)$ is called a Riemann matrix.

Definition 1.1.2. A Riemann theta function is defined by its Fourier series of the form

$$
\theta(z \mid B)=\sum_{N \in \mathbf{Z} g} \exp \left\{\frac{1}{2}\langle B N, N\rangle+\langle N, z\rangle\right\} .
$$

Here $z=\left(z_{1}, \ldots, z_{g}\right) \in \mathbf{C}^{g}$ is a complex vector. The diamond brackets denote the Euclidean scalar product: $\langle N, z\rangle=\sum_{i=1}^{g} N_{i} z_{i},\langle B N, N\rangle=\sum_{i, j=1}^{g} B_{i j} N_{i} N_{j}$. The summation in (1.1.1) is taken over the lattice of integer vectors $N=\left(N_{1}, \ldots, N_{g}\right)$. The general term of this series depends only on the symmetric part of the matrix $B$. From the obvious estimate $\operatorname{Re}\langle B N, N\rangle \leqslant$ $\leqslant-b\langle N, N\rangle, b>0$ (as $-b$ we can take the largest eigenvalue of the matrix $\operatorname{Re} B$ ), one derives easily that series (1.1.1) is absolutely convergent, uniformly on compact sets. Thus, the function $\theta(z \mid B)$ is analytic in the whole space $\mathbf{C}^{g}$.

Remark. We often use the abbreviated notation $\theta(z)=\theta(z \mid B)$ if the matrix $B$ is fixed.

Let $e_{1}, \ldots, e_{g}$ be the basis vectors in $\mathbf{C}^{g}$ with the coordinates

$$
\left(e_{k}\right)_{j}=\delta_{k j} ;
$$

we also introduce vectors $f_{1}, \ldots, f_{g}$, setting

$$
\left(f_{k}\right)_{j}=B_{k j} \quad(k, j=1, \ldots, g) .
$$


The vectors $f_{k}$ can also be written in the form

$$
f_{k}=B e_{k}
$$

where $B$ is the linear operator corresponding to $B_{k j}$.

Assertion 1.1.1. When the argument is shifted by the vectors $2 \pi i e_{k}$, the Riemann theta function $f_{k}$ is transformed according to the law

$$
\begin{gathered}
\theta\left(z+2 \pi i e_{k}\right)=\theta(z), \\
\theta\left(z+f_{k}\right)=\exp \left(-\frac{1}{2} B_{k k}-z_{k}\right) \theta(z) .
\end{gathered}
$$

Proof. The periodicity of (1.1.4) is obvious: the general term of (1.1.1) does not change under the shift $z \rightarrow z+2 \pi i e_{k}$. To prove (1.1.5) we change the summation index $N$ in (1.1.1), setting $N=M-e_{k}, M \in Z^{8}$. We have

$$
\begin{aligned}
& \theta\left(z+f_{k}\right)=\sum_{N \in \mathbf{Z} g} \exp \left\{\frac{1}{2}\langle B N, N\rangle+\left\langle N, z+f_{k}\right\rangle\right\}= \\
& =\sum_{M \in \mathbf{Z} g} \exp \left\{\frac{1}{2}\langle B M, M\rangle-\left\langle M, B e_{k}\right\rangle+\frac{1}{2}\left\langle B e_{k}, e_{k}\right\rangle+\right. \\
& \left.\quad \quad+\langle M, z\rangle+\left\langle M, f_{k}\right\rangle-\left\langle e_{k}, z\right\rangle-\left\langle e_{k}, f_{k}\right\rangle\right\}= \\
& =\exp \left\{-\frac{1}{2} B_{k k}-z_{k}\right\} \sum_{M \in \mathbf{Z} g} \exp \left\{\frac{1}{2}\langle B M, M\rangle+\langle M, z\rangle\right\}= \\
& \quad=\exp \left\{-\frac{1}{2} B_{k k}-z_{k}\right\} \theta(z) .
\end{aligned}
$$

This proves the assertion.

Thus, the function $\theta(z)$ is $g$-fold periodic with basis of periods $2 \pi i e_{1}, \ldots$, $2 \pi i e_{g}$. The vectors $f_{1}, \ldots, f_{g}$ are called its quasiperiods. Loosely speaking, we call the whole system of the vectors $\left(2 \pi i e_{k}, f_{1}\right)$ the periods of the $\theta$-function. Any vector of the form $2 \pi i N+B M$, where $N, M \in \mathbf{Z}^{g}$ are integer vectors, is a period of the Riemann $\theta$-function. From Assertion 1.1.1 we immediately get the transformation law:

$$
\theta(z+2 \pi i N+B M)=\exp \left[-\frac{1}{2}\langle B M, M\rangle-\langle M, z\rangle\right\} \theta(z) .
$$

The vectors of the form $2 \pi i N+B M$ constitute the period lattice.

We also define $\theta$-functions with characteristics. Let $\alpha$ and $\beta$ be arbitrary real $g$-dimensional vectors. We introduce a function $\theta[\alpha, \beta]$ (or $\theta[\alpha, \beta](z \mid B)$ in case we have to indicate the dependence on $B$ explicitly):

$$
\theta[\alpha, \beta](z)=\exp \left\{\frac{1}{2}\langle B \alpha, \alpha\rangle+\langle z+2 \pi i \beta, \alpha\rangle\right\} \theta(z+2 \pi i \beta+B \alpha) .
$$

For $\alpha=\beta=0$ we obtain the Riemann $\theta$-function: $\theta[0,0](z) \equiv \theta(z)$. Moreover, it follows from (1.1.6) that $\theta[N, M](z) \equiv \theta(z)$. Therefore, it suffices to consider the functions $\theta[\alpha, \beta](z)$ with characteristics $\alpha$ and $\beta$, 
such that $0<\alpha_{i}, \beta_{j}<1$. It is not hard to indicate the expansion of $\theta[\alpha, \beta](z)$ in a Fourier series. It has the form

$$
\text { (1.1.8) } \theta[\alpha, \beta](z)=\sum_{N \in \mathbf{Z} g} \exp \left\{\frac{1}{2}\langle B(N+\alpha), N+\alpha\rangle+\langle z+2 \pi i \beta, N+\alpha\rangle\right\} .
$$

By analogy with Assertion 1.1.1 we obtain the transformation law under a shift by a period of $\theta$-functions with characteristics. Omitting the calculations, we give the final formula

$$
\begin{aligned}
& \theta[\alpha, \beta](z+2 \pi i N+B M)= \\
= & \exp \left\{-\frac{1}{2}\langle B M, M\rangle-\langle z, M\rangle+2 \pi i(\langle\alpha, N\rangle-\langle\beta, M\rangle)\right\} \theta[\alpha, \beta](z) .
\end{aligned}
$$

When we multiply $\theta$-functions of the type (1.1.7), we obtain $\theta$-functions of higher orders. The transformation law of a $\theta$-function of order $n$ with characteristics $[\alpha, \beta]$, which we denote by $\theta_{n}[\alpha, \beta](z)$, under a shift by a period is as follows:

$$
\begin{aligned}
& \text { (1.1.10) } \quad \theta_{n}[\alpha, \beta](z+2 \pi i N+B M)= \\
& =\exp \left\{-\frac{n}{2}\langle B M, M\rangle-n\langle M, z\rangle+2 \pi i(\langle\alpha, N\rangle-\langle\beta, M\rangle)\right\} \theta_{n}[\alpha, \beta](z) .
\end{aligned}
$$

It is easy to show (but we omit this here) that the entire functions of $g$ variables $z_{1}, \ldots, z_{g}$ subject to the transformation law (1.1.10) form a linear space of dimension $n^{g}$. As basis $\theta$-function of order $n$ with characteristics $[\alpha, \beta]$ we can take, for example, the functions

$$
\theta\left[\frac{\alpha+\gamma}{n}, \beta\right](n z \mid n B),
$$

where the coordinates of $\gamma$ range independently over all values from 0 to $n-1$.

Definition 1.1.3. The characteristics $[\alpha, \beta]$ for which all the coordinates $\alpha_{i}, \beta_{i}$ are 0 or $\frac{1}{2}$ are called half-periods. A half-period $[\alpha, \beta]$ is said to be even if $4\langle\alpha, \beta\rangle \equiv 0(\bmod 2)$ and odd otherwise.

Assertion 1.1.2. The function $\theta[\alpha, \beta](z)$ is even or odd according as $[\alpha, \beta]$ is an even or odd half-period.

Proof. Under the substitution $z \rightarrow-z, N \mapsto-N-2 \alpha$ the general term of the series (1.1.8) is multiplied by

$$
\exp \{-\langle M+\beta, 4 \pi i \alpha\rangle\}=\exp \{4 \pi i\langle\alpha, \beta\rangle\} \text {. }
$$

The sign of this factor is completely determined by the parity of the number $4\langle\alpha, \beta\rangle$. This proves the assertion.

It is not hard to compute that there are precisely $2^{g-1}\left(2^{g}+1\right)$ even halfperiods and $2^{g-1}\left(2^{g}-1\right)$ odd ones. 


\section{$\S 2$. Theta functions of a single variable}

In the classical theory of elliptic functions (the case $g=1$ ) there occur only theta functions corresponding to half-periods. The Riemann matrix here is the single number $b=B_{11}, \operatorname{Re} b<0$. There are 4 half-periods: $(1 / 2,1 / 2),(1 / 2,0),(0,0),(0,1 / 2)$.

They correspond to 4 basic $\theta$-functions:

$$
(1.2 .2)\left\{\begin{array}{l}
i \theta_{1}(z) \equiv \theta\left[\frac{1}{2}, \frac{1}{2}\right](z)=\sum_{k \in \mathbf{Z}} \exp \left\{\frac{1}{2} b\left(k+\frac{1}{2}\right)^{2}+\left(\frac{2 k+1}{2}\right)(z+\pi i)\right\}, \\
\theta_{2}(z) \equiv \theta\left[\frac{1}{2}, 0\right](z)=\sum_{k \in \mathbf{Z}} \exp \left\{\frac{1}{2} b\left(\frac{2 k+1}{2}\right)^{2}+\frac{2 k+1}{2} z\right\}, \\
\theta_{3}(z) \equiv \theta[0,0](z)=\sum_{k \in \mathbf{Z}} \exp \left\{\frac{1}{2} b k^{2}+k z\right\}, \\
\theta_{4}(z) \equiv \theta\left[0, \frac{1}{2}\right](z)=\sum_{k \in \mathbf{Z}} \exp \left\{\frac{1}{2} b k^{2}+k(z+\pi i)\right\} .
\end{array}\right.
$$

For comparison with the literature we mention that the following notation is more generally accepted:

$$
b=2 \pi i \tau \quad(\operatorname{Im} \tau>0), \quad z=2 \pi i x .
$$

The first half-period is odd, the others are even. Consequently, the function $\theta_{1}(z)$ is odd and the others are even. The function $\theta_{1}(z)$ vanishes at all vertices of the period lattice $z=2 \pi i m+b n$. From this it is not hard to find the zeros of the remaining $\theta$-functions. In particular, $\theta_{3}(z)$ vanishes at all points $z=\pi i+b / 2+2 \pi i m+b n$. We claim that there are no other zeros. For convenience we take $\theta_{3}$. We need to prove that

$$
\frac{1}{2 \pi i} \oint_{C} d \log \theta_{3}(z)=1,
$$

where $C$ is the contour of the parallelogram spanned by the vectors $2 \pi i$ and $b$. The general transformation formulae (1.1.9) for $\theta_{3}(z)$ take the form

$$
\theta_{3}(z+2 \pi i)=\theta_{3}(z), \quad \theta_{3}(z+b)=\exp \left(-\frac{b}{2}-z\right) \theta_{3}(z) .
$$

We split the integral (1.2.3) into two:

$$
\begin{aligned}
\frac{1}{2 \pi i} \oint_{C} d \log \theta_{3}(z)=\frac{1}{2 \pi i} \int_{0}^{2 \pi i}\left[d \log \theta_{3}(z)\right. & \left.-d \log \theta_{3}(z+b)\right]+ \\
& +\frac{1}{2 \pi i} \int_{0}^{b}\left[d \log \theta_{3}(z+2 \pi i)-d \log \theta_{3}(z)\right] .
\end{aligned}
$$

From the relations (1.2.4) it follows that the second integral is zero, and the first integral has the form

as required.

$$
\frac{1}{2 \pi i} \int_{0}^{2 \pi i} d z=1
$$




\section{§3. On Abelian tori}

Assertion 1.3.1. The vectors $2 \pi i e_{1}, \ldots, 2 \pi i e_{g}, f_{1}, \ldots, f_{g}$ in the space $\mathbf{C}^{g}=\mathbf{R}^{2 g}$ defined by (1.1.2) and (1.1.3) are linearly independent over the field of real numbers.

Proof. We assume the contrary: that some linear combination of these vectors vanishes:

$$
2 \pi i \sum \lambda_{k} e_{k}+\sum \mu_{k} f_{k}=0, \quad \lambda_{k}, \mu_{k} \in \mathbf{R} .
$$

From (1.1.3) we obtain immediately that the real part of this equality has the form

$$
\operatorname{Re} B\left(\sum \mu_{k} e_{k}\right)=0 .
$$

Hence, all the $\mu_{k}$ are zero because the matrix $\operatorname{Re} B$ is non-singular. From (1.3.1) it then follows that all $\lambda_{k}$ are zero, which proves the assertion.

Let $\Gamma$ be the lattice generated by the vectors $\left(2 \pi i e_{k}, f_{1}\right)$. The vectors of $\Gamma$ have the form

$$
2 \pi i N+B M,
$$

where $N$ and $M$ are integer vectors. This is precisely the period lattice of the $\theta$-function in the sense of $\S 1$. It is convenient to associate with $\Gamma$ another geometric object-the quotient of $\mathbf{C}^{g}=\mathbf{R}^{\mathbf{2 g}}$ by this lattice. From Assertion 1.3.1 it follows immediately that this quotient $\mathbf{C}^{g} / \Gamma$ is a $2 g$-dimensional torus $T^{2 g}$. Moreover, $T^{2 g}$ has the natural structure of a complex compact Lie group, and the expression

$$
d s^{2}=-\sum_{k, l}(\operatorname{Re} B)_{k l}^{-1} d z_{k} d \bar{z}_{l}
$$

gives a Kähler metric on $T^{2 g}$, which is even a Hodge metric (see [4]). If $\alpha_{k}$ and $\beta_{j}$ are real coordinates in $\mathbf{R}^{2 g}=\mathbf{C}^{g}$, where $z=2 \pi i \beta+B \alpha$, then the imaginary part $\Omega$ of this metric has the form

$$
\Omega=\sum_{k=1}^{g} d \alpha_{k} \wedge d \beta_{k} .
$$

Such tori are called Abelian. We denote the Abelian torus $T^{2 g}$ constructed from the Riemann matrix $B=\left(B_{j k}\right)$ by $T^{28}(B)$.

The meromorphic functions on $T^{2 g}(B)$ are called Abelian functions. In other words, Abelian functions are $2 g$-fold periodic functions of $g$ complex variables. Thus, the quotient of two $\theta$-functions of the same order with the same characteristics is single-valued on $T^{2 g}(B)$ and is therefore an Abelian function. It is known that any Abelian function can be obtained in this way. A number of examples in which certain Abelian functions are expressed explicitly in terms of $\theta$-function, will be given in $\mathrm{Ch}$. II, $\S 4$. 
Let $\left(2 \pi i e_{j}^{\prime}, f_{k}^{\prime}\right)$ be another basis of $\Gamma$, where $f_{k}^{\prime}=B^{\prime} e_{k}^{\prime}$ and $B^{\prime}=\left(B_{j_{k}^{\prime}}^{\prime}\right)$ is another Riemann matrix. The transition from the basis $\left(2 \pi i e_{j}, f_{k}\right)$ to $\left(2 \pi i e_{j}^{\prime}, f_{k}^{\prime}\right)$ (and back) is given by an integer unimodular matrix

$$
\left\{\begin{aligned}
2 \pi i e_{i}^{\prime} & =\sum_{j} d_{i j} 2 \pi i e_{j}+\sum_{j} c_{i j} f_{j}, \\
f_{i}^{\prime} & =\sum_{j} b_{i j} 2 \pi i e_{j}+\sum_{j} a_{i j} f_{j},
\end{aligned}\right.
$$

where $a=\left(a_{i j}\right), b=\left(b_{i j}\right), c=\left(c_{i j}\right), d=\left(d_{i j}\right)$ are integer $(g \times g)$-matrices, and

$$
\operatorname{det}\left(\begin{array}{ll}
a & b \\
c & d
\end{array}\right)=1
$$

Here

$$
B^{\prime}=2 \pi i(a B+2 \pi i b)(c B+2 \pi i d)^{-1} .
$$

The requirement on $B^{\prime}$ to be a Riemann matrix imposes a strong restriction on the matrix $\left(\begin{array}{ll}a & b \\ c & d\end{array}\right)$; it must be symplectic:

$$
\left(\begin{array}{ll}
a & b \\
c & d
\end{array}\right) \in \operatorname{Sp}(g, \mathbf{Z})
$$

that is

$$
\left(\begin{array}{ll}
a & b \\
c & d
\end{array}\right)\left(\begin{array}{rr}
0 & 1 \\
-1 & 0
\end{array}\right)\left(\begin{array}{ll}
a^{t} & c^{t} \\
b^{t} & d^{t}
\end{array}\right)=\left(\begin{array}{rr}
0 & 1 \\
-1 & 0
\end{array}\right)
$$

(the symbol $t$ denotes transposition).

Two Riemann matrices $B$ and $B^{\prime}$ connected by transformations of the form (1.3.6), (1.3.7) are said to be equivalent. They determine the same Abelian tori $T^{2 g}(B)=T^{2 g}\left(B^{\prime}\right)$ (even with the same Hodge structures $\left.\left(1.3 .3^{\prime}\right)\right)^{(1)}$.

The following is the transformation law of $\theta$-functions under transformations of the form (1.3.6), (1.3.7):

where

$$
\begin{aligned}
& \theta\left[\alpha^{\prime}, \beta^{\prime}\right]\left(z^{\prime} \mid B^{\prime}\right)= \\
& \quad=k \sqrt{\operatorname{det} M} \exp \left\{\frac{1}{2} \sum_{i \leqslant j} z_{i} z_{j} \frac{\partial \log \operatorname{det} M}{\partial B_{i j}}\right\} \theta[\alpha, \beta](z \mid B),
\end{aligned}
$$

$$
\begin{gathered}
M=c B+2 \pi i d, \quad 2 \pi i z=z^{\prime} M \\
{\left[\alpha^{\prime}, \beta^{\prime}\right]=[\alpha, \beta]\left(\begin{array}{rr}
d & -b \\
-c & d
\end{array}\right)+\frac{1}{2} \operatorname{diag}\left[c d^{t}, a b^{t}\right] ;}
\end{gathered}
$$

$k$ is a constant independent of $z$ and $B$. Here the symbol diag means that one has to take the diagonal elements of the matrix $\left[c d^{t}, a b^{t}\right]$ (a proof of this transformation law is in [5]).

${ }^{(1)}$ The set $H_{g}$ of all $(g \times g)$-Riemann matrices is called the Siegel (left) half-plane. The group $\Lambda_{g}=\{\mathrm{Sp}(g, \mathrm{Z}) / t \pm 1\}$ acting on $H_{g}$ in accordance with (1.3.6) is the Siegel modular group. The factor group $H_{g} / \Lambda_{g}$ parametrizes the Abelian varieties $T^{2 g}$ with a Kähler metric of the type (1.3.3), (1.3.3'). 
Remark. Let $B=\left(B_{j k}\right)$ be a $(g \times g)$-Riemann matrix of block form $B=\left(\begin{array}{cc}B^{\prime} & 0 \\ 0 & B^{\prime \prime}\end{array}\right)$, where $B^{\prime}$ and $B^{\prime \prime}$ are $(k \times k)$ - and $(1 \times 1)$-Riemann matrices. Then the corresponding torus $T^{2 g}(B)$ splits into the direct product of two Abelian tori

$$
T^{2 g}(B)=T^{2 k}\left(B^{\prime}\right) \times T^{2 l}\left(B^{\prime \prime}\right)
$$

The corresponding $\theta$-function also splits into a product: if $z=\left(z_{1}, \ldots, z_{g}\right)$, $z^{\prime}=\left(z_{1}, \ldots, z_{k}\right)$, and $z^{\prime \prime}=\left(z_{k+1}, \ldots, z_{g}\right)$, then

$$
\theta(z \mid B)=\theta\left(z^{\prime} \mid B^{\prime}\right) \theta\left(z^{n} \mid B^{n}\right) \text {. }
$$

$\theta$-functions with characteristics are of a similar structure. We say that a Riemann matrix $B$ is decomposable if it can be brought to block form by transformations (1.3.6), (1.3.7). Correspondingly we use the term "decomposable Abelian torus" and "decomposable $\theta$-function" (formulae $(1.3 .10),(1.3 .11))$. For the opposite case of indecomposable tori $T^{2 g}(B)$ the following property holds: on $T^{2 g}(B)$ there are no (non-constant) Abelian functions depending on a smaller number of variables. Equivalent is the assertion: if $f(z)$ is an Abelian function on an indecomposable torus and its derivative with respect in some direction vanishes, $\sum_{i=1}^{g} U_{i} \frac{\partial f}{\partial z_{i}}=0$, then
$f=$ const.

Returning to Abelian tori, we note the following important theorem: any Abelian torus is an algebraic variety (Lefschetz). We do not prove this theorem in general (the proof is based on the theory of $\theta$-functions; see [11]), but consider only the case $g=1$. In this case any torus $T^{2}$ is determined by its pair of periods $2 \omega, 2 \omega^{\prime}$ (we assume that $\operatorname{Im} \omega^{\prime} / \omega>0$ ). We define the Weierstrass elliptic function $8(z)$, setting

$$
\wp(z)=\frac{1}{z^{2}}+\sum_{m^{2}+n^{2} \neq 0}\left[\frac{1}{\left(z-2 m \omega-2 n \omega^{\prime}\right)^{2}}-\frac{1}{\left(2 m \omega+2 n \omega^{\prime}\right)^{2}}\right] .
$$

It is not hard to verify that the series (1.3.12) converges uniformly on every compact set in $\mathbf{C} \backslash\left\{2 m \omega+2 n \omega^{\prime}\right\}$, so that it is a meromorphic function of $z$ having double poles on each vertex of the lattice. Obviously, this function is doubly periodic:

$$
\wp\left(z+2 m \omega+2 n \omega^{\prime}\right)=\wp(z), \quad n, m \in \mathbf{Z} .
$$

The derivative $\wp^{\prime}(z)$ is also doubly periodic. Let

$$
\left\{\begin{array}{l}
g_{2}=60 \sum_{m^{2}+n^{2} \neq 0} \frac{1}{\left(2 m \omega+2 n \omega^{\prime}\right)^{4}}, \\
g_{3}=140 \sum_{n^{2}+m^{2} \neq 0} \frac{1}{\left(2 m \omega+2 n \omega^{\prime}\right)^{6}} .
\end{array}\right.
$$


Then the Laurent expansions of $\delta^{\circ}(z)$ and $\delta^{\prime}(z)$ in a neighbourhood of $z=0$ have the form

$$
\begin{gathered}
\wp(z)=\frac{1}{z^{2}}+\frac{g_{2} z^{2}}{20}+\frac{g_{3} z^{4}}{28}+\ldots, \\
\wp^{\prime}(z)=-\frac{2}{z^{3}}+\frac{g_{2} z}{10}+\frac{g_{3} z^{3}}{7}+\ldots
\end{gathered}
$$

From these expansions it follows that the function $\left(8^{\circ}\right)^{2}-\left[48^{3}-g_{2} 8-g_{3}\right]$ does not have a pole at the origin. Since it is doubly periodic, this function is regular on the whole complex plane, consequently, it is a constant. It is not difficult to see that this constant is zero. We deduce that the Weierstrass function $\delta^{\circ}(z)$ satisfies the differential equation

$$
\left(8^{\prime}\right)^{2}=48^{3}-g_{2} 8-g_{3}
$$

Assertion 1.3.2. The mapping $x(z)=\varnothing^{\prime}(z), y(z)=\wp^{\prime}(z)$ establishes an isomorphism of the complex torus $T^{2}=\mathrm{C} /\left\{2 m \omega+2 n \omega^{\prime}\right\}$ with the Riemann surface of the algebraic function

$$
y^{2}=4 x^{3}-g_{2} x-g_{3} .
$$

Proof. We construct the inverse mapping from the complex algebraic curve (1.3.18) into the torus $T^{2}$. Let $P=(x, y)$ be a point of the Riemann surface (1.3.18). We set

$$
z=z(P)=\int_{\infty}^{P} \frac{d x}{y}=\int_{\infty}^{P} \frac{d x}{\sqrt{4 x^{3}-g_{2} x-g_{3}}} .
$$

The path of integration in (1.3.19) lies on the Riemann surface (1.3.18). This path is unique, up to addition of an integral linear combination of the base cycles $a$ and $b$ on (1.3.18) (Figure 1; here $4 x_{i}^{3}-g_{2} x_{i}-g_{3}=0$; the dotted line shows the part of the cycle $b$, lying

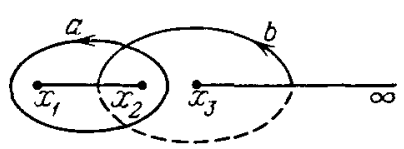

Fig. 1. Riemann surface of (1.3.18) on the "lower" sheet):

$$
\text { (1.3.20) } z(P) \sim z(P)+m \oint_{a} \frac{d x}{y}+n \oint_{b} \frac{d x}{y} \text {. }
$$

It is not difficult to verify that

Thus, the mapping (1.3.19) carries the curve (1.3.18) into the torus $T^{2}$. Obviously, the mappings $z \mapsto P=\left(\gamma^{\circ}(z), 8^{\prime}(z)\right)$ and $P \mapsto \int_{\infty}^{p} \frac{d x}{y}$ are inverse to
each other. This proves the assertion. Remark. Let us indicate an explicit connection between the Weierstrass function $\wp(z)$ and the $\theta$-functions of one variable considered in $\S 2$. There is the formula

$$
\wp(z)=-\frac{\partial^{2}}{\partial z^{2}} \log \theta_{1}(z)+c,
$$


where $c$ is a constant. This is obvious in view of the periodicity of the righthand side and the information obtained in $\$ 2$ on the location of the zeros of $\theta_{1}(z)$. From (1.3.22) and (1.3.17) it follows that $\theta_{1}(z)$ satisfies a thirdorder differential equation. Later we consider the differential relations for multidimensional $\theta$-functions (see Chapters 3 and 4).

\section{$\S 4$. Addition theorems for $\theta$-functions}

Theta functions are connected by a complicated system of algebraic relations, the so-called addition theorems. All of them are relations between formal Fourier series. We quote the two most important addition theorems. ${ }^{(1)}$

From a Riemann matrix $B$ we construct two sets of $\theta$-functions:

(1.4.1) $\quad \theta[\alpha, \beta](z) \equiv \theta[\alpha, \beta](z \mid B), \quad \hat{\theta}[\alpha, \beta](z) \equiv \theta[\alpha, \beta](z \mid 2 B)$.

Addition Theorem 1.4.1. Let $\alpha, \beta, \gamma$ and $\varepsilon$ be arbitrary real g-dimensional vectors. Then

$$
\begin{aligned}
& \theta[\alpha, \gamma]\left(z_{1}+z_{2}\right) \theta[\beta, \varepsilon]\left(z_{1}-z_{2}\right)= \\
& \quad=\sum_{2 \delta \in\left(\mathbf{Z}_{2}\right) g} \hat{\theta}\left[\frac{\alpha+\beta}{2}+\delta, \gamma+\varepsilon\right]\left(2 z_{1}\right) \hat{\theta}\left[\frac{\alpha-\beta}{2}+\delta, \gamma-\varepsilon\right]\left(2 z_{2}\right) .
\end{aligned}
$$

It suffices to prove this for the case $\alpha=\beta=\gamma=\varepsilon=0$ (the general case reduces to this one via (1.1.7)). Then (1.4.2) takes the form

$$
\theta(z+w) \theta(z-w)=\sum_{2 \delta \in\left(\mathbf{Z}_{2}\right)^{g}} \hat{\theta}[\delta, 0](2 z) \hat{\theta}[\delta, 0](2 w) .
$$

First we consider the case $g=1$. Then $\left(1.4 .2^{\prime}\right)$ can be written as where

(1.4.3) $\theta(z+w) \theta(z-w)=\hat{\theta}(2 z) \hat{\theta}(2 w)+\hat{\theta}\left[\frac{1}{2}, 0\right](2 z) \hat{\theta}\left[\frac{1}{2}, 0\right](2 u)$,

$$
\begin{array}{r}
\theta(z)=\sum_{k} \exp \left(\frac{1}{2} b k^{2}+k z\right), \quad \hat{\theta}(z)=\sum_{k} \exp \left(b k^{2}+k z\right), \\
\hat{\theta}\left[\frac{1}{2}, 0\right](z)=\sum_{k} \exp \left[b\left(k+\frac{1}{2}\right)^{2}+\left(k+\frac{1}{2}\right) z\right], \quad b=B_{11} .
\end{array}
$$

The left-hand side of (1.4.3) therefore has the form

$$
\sum_{k, l} \exp \left[\frac{1}{2} b\left(k^{2}+l^{2}\right)+k(z+w)+l(z-w)\right] .
$$

We introduce new summation indices $m, n$, setting

$$
m=\frac{k+l}{2}, \quad n=\frac{k-l}{2} .
$$

The numbers $m$ and $n$ are both either integers or half-integers. In these variables (1.4.4) takes the form

$$
\theta(z+w) \theta(z-w)=\sum \exp \left[b m^{2}+2 m z+b n^{2}+2 n w\right] .
$$

${ }^{(1)}$ Relations for theta functions of an entirely different type can be found in [43] 
We split this sum into two parts, the first part containing the terms with $m$ and $n$ integers, and the second half-integers. In the second part we change $m$ to $m+\frac{1}{2}$ and $n$ to $n+\frac{1}{2}$. Then $m$ and $n$ are integers, and (1.4.6) takes the form

$\sum_{m, n \in \mathbf{Z}} \exp \left[b m^{2}+2 m z\right] \exp \left[b n^{2}+2 n w\right]+$

$$
\begin{array}{r}
+\sum_{m, n \in \mathbf{Z}} \exp \left[b\left(m+\frac{1}{2}\right)^{2}+2\left(m+\frac{1}{2}\right) z\right] \exp \left[b\left(n+\frac{1}{2}\right)^{2}+2\left(n+\frac{1}{2}\right) w\right]= \\
=\hat{\theta}(2 z) \hat{\theta}(2 w)+\hat{\theta}\left[\frac{1}{2}, 0\right](2 z) \hat{\theta}\left[\frac{1}{2}, 0\right](2 w) .
\end{array}
$$

Thus, the theorem is proved in this special case. In the general case $g \geqslant 1$ we have to repeat this argument for each coordinate separately.

Addition Theorem 1.4.2. Let $\left[m_{i}\right]=\left[m_{i}^{\prime}, \quad m_{i}^{\prime \prime}\right] \quad(i=1,2,3,4)$ be arbitrary real $2 \mathrm{~g}$-dimensional vectors. Then

$$
\begin{aligned}
& \theta\left[m_{1}\right]\left(z_{1}\right) \theta\left[m_{2}\right]\left(z_{2}\right) \theta\left[m_{3}\right]\left(z_{3}\right) \theta\left[m_{4}\right]\left(z_{4}\right)= \\
& =\frac{1}{2^{g}} \sum_{2 a \in\left(\mathbf{Z}_{2}\right)^{2 g}} \exp \left(-4 \pi i\left\langle m_{1}^{\prime}, a^{\prime \prime}\right\rangle\right) \theta\left[n_{1}+a\right]\left(w_{1}\right) \ldots \theta\left[n_{4}+a\right]\left(w_{4}\right),
\end{aligned}
$$

where $a=\left(a^{\prime}, a^{\prime \prime}\right)$,

$$
\begin{gathered}
\left(z_{1}, \ldots, z_{4}\right)=\left(w_{1}, \ldots, w_{4}\right) T, \quad T=\frac{1}{2}\left(\begin{array}{rrrr}
1 & 1 & 1 & 1 \\
1 & 1 & -1 & -1 \\
1 & -1 & 1 & -1 \\
1 & -1 & -1 & 1
\end{array}\right) \\
{\left[n_{i}\right]=\left[n_{i}^{\prime}, n_{i}^{\prime \prime}\right], \quad\left(m_{1}, \ldots, m_{4}\right)=\left(n_{1}, \ldots, n_{4}\right) T}
\end{gathered}
$$

(Each 1 in $T$ is the unit $(g \times g)$-or $(2 g \times 2 g)$-matrix).

Again we give the proof for the simplest case $g=1$ only. The matrix $T$ has two important properties: it is symmetric and orthogonal. The general term of the sum on the left-hand side has the form

$$
\begin{aligned}
\exp \left\{\frac{1}{2} b\right. & {\left[\left(k_{1}+m_{1}^{\prime}\right)^{2}+\ldots+\left(k_{4}+m_{4}^{\prime}\right)^{2}\right]+} \\
& \left.+\left(k_{1}+m_{1}^{\prime}\right)\left(z_{1}+2 \pi i m_{1}^{\prime \prime}\right)+\ldots+\left(k_{4}+m_{4}^{\prime}\right)\left(z_{4}+2 \pi i m_{4}^{\prime \prime}\right)\right\},
\end{aligned}
$$

where $k_{1}, \ldots, k_{4}$ are integers. We introduce new summation variables $l_{1}, \ldots, l_{4}$, setting

$$
\left(l_{1}, \ldots, l_{4}\right)=\left(k_{1}, \ldots, k_{4}\right) T .
$$

We call the set $k_{1}, \ldots, k_{4}$ even if the sum $k_{1}+\ldots+k_{4}$ is even, otherwise odd. For an even set $k_{1}, \ldots, k_{4}$ all the numbers $l_{1}, \ldots, l_{4}$ are integers, and for an odd one they are half-integers. Taking (1.4.8), (1.4.10), and the orthogonality and symmetry of $T$ into account, we obtain for (1.4.9) in the new variables:

$$
\begin{aligned}
\exp \left\{\frac{1}{2} b\right. & {\left[\left(l_{1}+n_{1}^{\prime}\right)^{2}+\ldots+\left(l_{4}+n_{4}^{\prime}\right)^{2}\right]+} \\
& \left.+\left(l_{1}+n_{1}^{\prime}\right)\left(w_{1}+2 \pi i n_{1}^{\prime \prime}\right)+\ldots+\left(l_{4}+n_{4}^{\prime}\right)\left(w_{4}+2 \pi i n_{4}^{\prime \prime}\right)\right\} .
\end{aligned}
$$


We divide the sum of exponentials of the form (1.4.11) into two parts: $\Sigma_{(1)}$ and $\Sigma_{(2)} . \Sigma_{(1)}$ includes only the integers $l_{1}, \ldots, l_{4}$ and $\Sigma_{(2)}$ only the halfinteger ones. In $\Sigma_{(2)}$, as in the proof of the preceding theorem, we make the change of variables $l_{i} \mapsto l_{i}+\frac{1}{2}$, after which both $\Sigma_{(1)}$ and $\Sigma_{(2)}$ include only integer summation indices $l_{1}, \ldots, l_{4}$. We remark that the set $l_{1}, \ldots, l_{4}$ is necessarily even, since $k_{1}, \ldots, k_{4}$ are integers and $\left(k_{1}, \ldots, k_{4}\right)=\left(l_{1}, \ldots . l_{4}\right) T$. Thus, the summation in $\Sigma_{(1)}$ and $\Sigma_{(2)}$ is only over even sets $l_{1}, \ldots, l_{4}$. We sum over all the sets $l_{1}, \ldots, l_{4}$ and to compensate for the inclusion of the odd sets we also add the sums $\Sigma_{1}^{\prime}(1)$ and $\Sigma_{(2)}^{\prime}$, in which we have made the change of variables $n_{i}^{\prime \prime} \mapsto n_{i}^{\prime \prime}+\frac{1}{2}(i=1, \ldots, 4)$ in the general term; also, to the whole exponent of the exponential we add the term $-\left(n_{1}^{\prime}+\ldots+\right.$ $\left.+n_{4}^{\prime}\right) \pi i=-4 \pi i m_{1}^{\prime} / 2$. . After these transformations the even terms in $\Sigma_{(1)}+\Sigma_{(1)}^{\prime}$ are doubled, and the odd ones cancel each other. The same holds for the sum $\Sigma_{(2)}+\Sigma_{(2)}^{\prime}$. Thus, we obtain

$$
\theta\left[m_{1}\right]\left(z_{1}\right) \ldots \theta\left[m_{4}\right]\left(z_{4}\right)=\frac{1}{2}\left(\Sigma_{(1)}+\Sigma_{(1)}^{\prime}+\Sigma_{(2)}+\Sigma_{(2)}^{\prime}\right) .
$$

This coincides precisely with (1.4.7) for $g=1$. In the general case the proof is similar, only we have to repeat these arguments for each coordinate.

Setting $z_{1}=u+v, z_{2}=u-v, z_{3}=z_{4}=0$ in (1.4.7), we have $w_{1}=w_{2}=u, w_{3}=w_{4}=v$. The second addition theorem in this special case takes the form

$$
\begin{aligned}
=\frac{1}{2^{g}} \sum_{2 a \in\left(\mathbf{Z}_{2}\right)^{2 g}} \exp \left[-4 \pi i\left\langle m_{1}^{\prime}, a^{\prime \prime}\right\rangle\right] \theta\left[n_{1}\right. & +a](u) \theta\left[n_{2}+a\right](u) \times \\
& \times \theta\left[n_{3}+a\right](v) \theta\left[n_{4}+a\right](v),
\end{aligned}
$$

where the connection of the characteristics $n_{1}, \ldots, n_{4}$ with $m_{1}, \ldots, m_{4}$ is given by $\left(1.4 .8^{\prime}\right)$.

\section{ChAPTER ॥}

THETA FUNCTIONS OF RIEMANN SURFACES. THE JACOBI INVERSION PROBLEM

$\S 1$. Periods of Abelian differentials on Riemann surfaces.
Jacobi varieties

Let $\Gamma$ be a compact Riemann surface ${ }^{(1)}$ of genus $g \geqslant 1$. If $\Gamma$ is the Riemann surface of an algebraic function $w=w(z)$ given by an equation

$$
R(w, z)=w^{n}+a_{1}(z) w^{n-1}+\ldots+a_{n}(z)=0,
$$

(1) From here on only compact Riemann surfaces occur, and we do not state this every time. 
where $R(w, z)$ is a polynomial, then the affine part of $\Gamma$ coincides with the complex algebraic curve $(2.1 .1)$ in $C^{2}$, provided that this curve is non-singular (smooth). The important example for us is the class of hyperelliptic curves, which are given by equations

$$
w^{2}=P_{2 g+1}(z)
$$

or

$$
w^{2}=P_{2 g+2}(z)
$$

where $P_{2 g+1}(z)$ and $P_{2 g+2}(z)$ are polynomials of degrees $2 g+1$ and $2 g+2$, respectively, without multiple roots (and in either case the genus of the corresponding Riemann surface is $g$ ). We mention that every Riemann

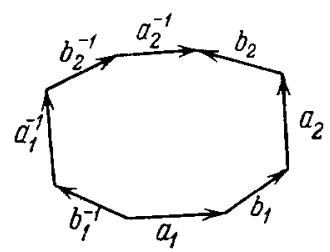
surface of genus $g=1$ or $g=2$ has the form (2.1.2) (or (2.1.3); see [3]); for $g=3$ this is no longer so.

Topologically, a surface of genus $g$ is a sphere with $g$ handles attached. In the 1-dimensional homology group $H_{1}(\Gamma)=\mathbf{Z}+\ldots+\mathbf{Z}$ (2g factors) one can choose a basis of cycles (closed contours) $a_{1}, \ldots, a_{g}, b_{1}, \ldots, b_{g}$ with the following intersection indices:

$$
a_{i} \circ a_{j}=b_{i} \circ b_{j}=0, \quad a_{i} \circ b_{j}=\delta_{i j} \quad(i, j=1, \ldots, g) .
$$

This basis has the property that under cutting along these cycles, $\Gamma$ becomes a $4 g$-gon, which we denote by $\widetilde{\Gamma}$ (see Fig. 2 for $g=2$ ). Each cycle goes to a pair of sides $a_{i}, a_{i}^{-1}, b_{i}, b_{i}^{-1}$ of $\widetilde{\Gamma}$, which are identified on $\Gamma$.

Differential 1-forms $\omega=a d x+b d y=\alpha d z+\beta d \bar{z}$ (where $z=x+i y$ is a complex local coordinate on $\Gamma$ ) are simply called differentials.

Definition 2.1.1. A differential $\omega$ is said to be Abelian of the first kind or holomorphic if in a neighbourhood of any point it can be written in the form

$$
\omega=f(z) d z
$$

where $f(z)$ is an analytic function and $z$ a local coordinate.

For example, on the hyperelliptic surface (2.1.2) the differentials $\omega_{1}, \ldots, \omega_{g}$ of the form

$$
\omega_{k}=\frac{z^{k-1}}{\sqrt{P_{2 g+1}(z)}} d z \quad(k=1, \ldots, g)
$$

are all holomorphic.

A holomorphic differential is closed: $d \omega=0$. Its complex conjugate differential $\bar{\omega}=\overline{f(z)} d \bar{z}$ is also closed. For any closed differential $\omega$ on $\Gamma$ its periods over the cycles $a_{1}, \ldots, a_{g}, b_{1}, \ldots, b_{g}$ are defined as follows:

$$
\oint_{a_{i}} \omega=A_{i}, \quad \oint_{b_{i}} \omega=B_{i} \quad(i=1, \ldots, g) .
$$


We fix a point $P_{0}$ not lying on the cycles $a_{i}, b_{j}$. Then we can define the function

$$
f(P)=\int_{P_{0}}^{P} \omega
$$

which is single-valued on the cut surface $\widetilde{\Gamma}$. The following lemma is useful.

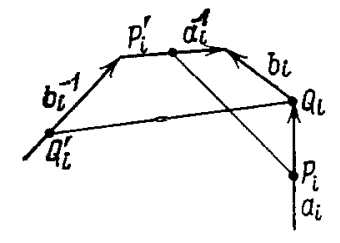

Fig. 3.

Lemma 2.1.1. Let $\omega$ and $\omega^{\prime}$ be two closed differentials on $\Gamma$; let $A_{i}, A_{i}^{\prime}, B_{i}, B_{i}^{\prime}$ be the set of their a-and b-periods; let $f(P)=\int_{P_{0}}^{P} \omega$;

then

$$
\iint_{\Gamma} \omega \wedge \omega^{\prime}=\oint_{\partial \widetilde{\Gamma}} f \omega^{\prime}=\sum_{i=1}^{g}\left(A_{i} B_{i}^{\prime}-A_{i}^{\prime} B_{i}\right)
$$

Here $\partial \widetilde{\Gamma}$ is the boundary of $\widetilde{\Gamma}$, oriented in the positive direction.

Proof. The equality $\iint_{\Gamma} \omega \wedge \omega^{\prime}=\oint_{\partial \widetilde{\Gamma}} f \omega^{\prime}$ is obvious from Stokes' formula.
Further,

$$
\oint_{\partial \widetilde{\Gamma}} f \omega^{\prime}=\sum_{i=1}^{g}\left(\int_{a_{i}}+\int_{a_{i}^{-1}}\right) f \omega^{\prime}+\sum_{i=1}^{g}\left(\int_{b_{i}}+\int_{b_{i}^{-1}}\right) f \omega^{\prime} .
$$

From Fig. 3 it is clear that $f\left(P_{i}\right)-f\left(P_{i}^{\prime}\right)=\int_{P_{i}^{\prime}}^{P_{i}} \omega=-B_{i}$ (the cycle $P_{i} P_{i}^{\prime}$ on $\Gamma$ is homologous to $\left.b_{i}\right)$ and that $f\left(Q_{i}\right)-f\left(Q_{i}^{\prime}\right)=A_{i}$ for a similar reason. Therefore, the sum (2.1.10) can be rewritten in the form

$$
\oint_{\widetilde{\widetilde{T}}} f \omega^{\prime}=\sum_{i=1}^{g}\left(-B_{i}\right) \oint_{a_{i}} \omega^{\prime}+\sum_{i=1}^{g} A_{i} \oint_{b_{i}} \omega^{\prime}=\sum_{i=1}^{g}\left(A_{i} B_{i}^{\prime}-A_{i}^{\prime} B_{i}\right) .
$$

This proves the Lemma.

Applying this lemma to a pair $\omega, \bar{\omega}$, where $\omega$ is a holomorphic differential and $\bar{\omega}$ its complex conjugate, we obtain the following corollary.

Corollary 1. Let $\omega$ be a non-zero holomorphic differential on $\Gamma$. Then its $a$ - and b-periods $A_{k}, B_{k}$ satisfy the inequality

$$
\operatorname{Im} \sum_{k=1}^{g} A_{k} \bar{B}_{k}<0
$$

(Im denotes the imaginary part).

From this we immediately obtain the next result.

Corollary 2. If all the a-periods $A_{1}, \ldots, A_{g}$ of a holomorphic differential $\omega$ vanish, then $\omega \equiv 0$. 
For a hyperelliptic Riemann surface we have produced above (see 2.1.6)) a set of $g$ holomorphic differentials $\omega_{1}, \ldots, \omega_{g}$. For any Riemann surface $\Gamma$ we can also construct $g$ linearly independent holomorphic differentials $\omega_{1}, \ldots, \omega_{g}$. For a proof of their existence, see [1]. From this and Corollary 2 we obtain a further corollary.

Corollary 3. The space of holomorphic differentials on a Riemann surface of genus $\mathrm{g}$ is g-dimensional.

Let $\eta_{1}, \ldots, \eta_{g}$ be a set of linearly independent holomorphic differentials on a Riemann surface $\Gamma$. Then the matrix

$$
A_{j k}=\oint_{a_{k}} \eta_{j}
$$

of their $a$-periods is non-singular. For otherwise some non-trivial linear combination $\eta=c_{1} \eta_{1}+\ldots+c_{g} \eta_{g}$ would have zero $a$-periods, which contradicts Corollary 2. Therefore, one can choose another basis of holomorphic differentials $\omega_{1}, \ldots, \omega_{g}$,

$$
\omega_{j}=\sum_{k=1}^{g} c_{j k} \eta_{k} \quad(j=1, \ldots, g),
$$

normalized by the conditions

$$
\oint_{a_{k}} \omega_{j}=2 \pi i \delta_{j k} \quad(j, k=1, \ldots, g) .
$$

Here $\left(c_{j k}\right)$ is a non-singular matrix of the form $\left(c_{j k}\right)=2 \pi i\left(A_{j k}\right)^{-1}$. This basis of holomorphic differentials is called canonically dual to the basis of cycles $a_{1}, \ldots, a_{g}, b_{1}, \ldots, b_{g}$.

From the canonical basis $\omega_{1}, \ldots, \omega_{g}$ we construct the matrix of $b$-periods

$$
B_{j k}=\oint_{b_{k}} \omega_{j} \quad(j, k=1, \ldots, g) .
$$

Theorem 2.1.1. $B_{j k}$ is a Riemann matrix.

Proof. Its symmetry follows from Lemma 2.1.1 applied to the pair of basis differentials $\omega=\omega_{j}, \omega^{\prime}=\omega_{k}$ (obviously, $\omega \wedge \omega^{\prime}=0$ if $\omega$ and $\omega^{\prime}$ are both holomorphic). To prove that the real part $\operatorname{Re} B_{j k}$ is negative definite we consider a non-trivial linear combination

$$
\omega=\sum_{j=1}^{g} c_{j} \omega_{j},
$$

where all the coefficients $c_{1}, \ldots, c_{g}$ are real. The periods of this holomorphic differential are

$$
A_{k}=2 \pi i c_{k}, \quad B_{k}=\sum_{j=1}^{g} c_{j} B_{j k} \quad(k=1, \ldots, g) .
$$


Applying (2.1.11) to it, we obtain

$$
2 \pi \sum_{j, k=1}^{g} \operatorname{Re} B_{j k} c_{k} c_{j}=\operatorname{Im} \sum_{k=1}^{g} A_{k} \bar{B}_{k}<0 .
$$

This proves the theorem.

Thus, for each Riemann surface $\Gamma$ of genus $g$ and each basis of cycles $a_{1}, \ldots, a_{g}, b_{1}, \ldots, b_{g}$ on $\Gamma$ we have constructed a Riemann matrix $B_{j k}$.

Definition 2.1.2. The Abelian torus $T^{2 g}(B)$ constructed from the matrix $B=\left(B_{j k}\right)$ of periods of holomorphic differentials on the Riemann surface $\Gamma$ is called the Jacobi variety (or Jacobian) of this surface, and is denoted by $J(\Gamma)$.

$$
J(\Gamma)=T^{2} \boldsymbol{g}(B)=[\mathbb{B} /\{2 \pi i N+B M\} .
$$

The $\theta$-functions $\theta[\alpha, \beta](z \mid B)$ constructed from the matrix are called the $\theta$-functions of the Riemann surface $\Gamma$.

If $a_{1}^{\prime}, \ldots, a_{g}^{\prime}, b_{1}^{\prime}, \ldots, b_{g}^{\prime}$ is another basis of cycles with intersection indices (2.1.4), then the transition matrix from the old to the new basis is integral and symplectic (the intersection index is a skew-symmetric nondegenerate form on $H_{1}(\Gamma)$, given in both cases the same matrix). Thus, a change of bases in $H_{1}(\Gamma)$ leads to an equivalent Riemann matrix $B_{j k}^{\prime}$ (connected with the old one by transformations of the form (1.3.6), (1.3.7)). Therefore, the definition of the Jacobi variety $J(\Gamma)$ does not depend on the choice of a basis of cycles. The corresponding $\theta$-function also does not change substantially in view of the transformation law (1.3.8).

Remark. A natural question arises: what Riemann matrices $B_{j k}$ are matrices of periods of holomorphic differentials on a Riemann surface? One restriction on such matrices $B=\left(B_{j k}\right)$ is known: they cannot have block form $B=\left(\begin{array}{cc}B^{\prime} & 0 \\ 0 & B^{\prime \prime}\end{array}\right)$, where $B^{\prime}$ and $B^{\prime \prime}$ are again Riemann matrices of a smaller genus (more precisely, they cannot be reduced to block form by transformations (1.3.6), (1.3.7)). For the genera $g=1,2,3$ there are no other restrictions: any Riemann matrix (in general position) is a period matrix of the holomorphic differentials on a Riemann surface. For $g=1$ this was proved in Ch. I, $\S 3$; for $g=2$ and 3 it will be proved in Ch. IV. From dimension arguments it is clear that for $g \geqslant 4$ this is no longer true. It is known that the set of all (non-isomorphic) Riemann surfaces of genus $g>1$ depends on $3 g-3$ complex parameters. This is also the dimension of the set of period matrices on these surfaces. However, the Riemann matrices form an open cone in the complex space of dimension $g(g+1) / 2$. Therefore, even for $g=4$ there must be a relation on the period matrix $B_{j k}$ (found by Schottky [26]). For large genera such relations have so far not been written out in a good form. We shall return to this question in Chapter IV.

Now suppose that $\omega$ is a meromorphic differential on $\Gamma$ (singularities of the type of poles are admitted). We assume that its poles do not lie on the cycles $a_{i}, b_{j}$. By adding a suitable holomorphic differential we can achieve that $\omega$ has zero $a$-periods; the location of the poles and the 
corresponding principal parts of $\omega$ do not change. Such a normalization together with a specification of the poles and principal parts determines the meromorphic differential $\omega$ uniquely (otherwise the difference of two such differentials would be a holomorphic differential with zero $a$-periods).

The sum of the residues on $\Gamma$ of any meromorphic differential is zero. Therefore, it can be represented as a linear combination (up to addition of a holomorphic differential) of the following basic meromorphic differentials: ${ }^{(1)}$

a) Abelian differentials of the second kind. These are differentials $\omega_{Q}^{(n)}$ with a single pole at $Q$ of multiplicity $n+1$ and with principal part of the form

$$
\omega_{Q}^{(n)}=\frac{d z}{z^{n+1}}+\cdots \quad(n \geqslant 1) .
$$

b) Abelian differentials of the third kind $\omega_{P Q}$. These have a pair of simple poles at $P$ and $Q$ with residues +1 and -1 , respectively.

We recall that these differentials are uniquely determined by the normalization

$$
\begin{aligned}
& \oint_{a_{i}} \omega_{Q}^{(n)}=0 \quad(i=1, \ldots, g), \\
& \oint_{a_{i}} \omega_{P Q}=0 \quad(i=1, \ldots, g) .
\end{aligned}
$$

We now list the relations between the $b$-periods of these differentials that will be needed later on:

Lemma 2.1.2. The following relations hold:

$$
\begin{gathered}
\oint_{b_{i}} \omega_{Q}^{(n)}=\frac{1}{n !} \frac{d^{n-1} j_{i}(Q)}{d z^{n-1}} \quad(i=1, \ldots, g ; n=1,2, \ldots), \\
\oint_{b_{i}} \omega_{P Q}=\int_{Q}^{P} \omega_{i} \quad(i=1, \ldots, g),
\end{gathered}
$$

where $\omega_{Q}^{(n)}$ and $\omega_{P Q}$ are the normalized differentials of the second and third kind introduced above, and $\omega_{1}, \ldots, \omega_{g}$ are basis holomorphic differentials, given by $\omega_{i}=f_{i}(z) d z$ in a neighbourhood of $Q$.

The proof of the relations (2.1.21) and (2.1.22) is obtained by integrating the expressions $A_{i} \omega_{Q}^{(n)}$ and $A_{i} \omega_{P Q}$, where $A_{i}(P)=\int_{P_{0}}^{P} \omega_{i}$, over the boundary of the domain obtained from the $4 g$-gon $\widetilde{\Gamma}$ by removing the set of edges going from the initial point $P_{0}$ to the poles of the differentials. We omit the computations, which are similar to those in the proof of Lemma 2.1.1.

\footnotetext{
(1) We do not prove here the existence of such differentials (see [1]). For a hyperelliptic surface it is not difficult to produce them by explicit formulae. In the general case, the existence of such differentials can be deduced, for example, from the Riemann-Roch theorem (see below §3).
} 


\section{§2. Abel's theorem}

Let $\Gamma$ be a Riemann surface of genus $g$, and $J(\Gamma)$ its Jacobi variety. We define the Abel mapping $A(P)=\left(A_{1}(P), \ldots, A_{g}(P)\right)$,

$$
A: \Gamma \rightarrow J(\Gamma),
$$

setting

$$
A_{k}(P)=\int_{P_{0}}^{p} \omega_{k} \quad(k=1, \ldots, g) .
$$

Here $P_{0}$ is a fixed point; the path of integration from $P_{0}$ to $P$ is chosen to be the same for all $k$. If we choose another path of integration from $P_{0}$ to $P$ in (2.2.1), then we have to add $\oint_{\gamma} \omega_{k}$ to the integral on the right, where $\gamma$ is a closed contour (cycle). The cycle $\gamma$ can be represented as an integral linear combination of the basis cycles:

$$
\gamma=\sum_{j} n_{j} a_{j}+\sum_{j} m_{j} b_{j}
$$

Therefore, the added term on the right-hand side of (2.2.1) has the form

$$
\oint_{\gamma} \omega_{k}=2 \pi i n_{k}+\sum_{j} B_{j k} m_{j},
$$

but this is the $k$-th component of some vector of the lattice $\{2 \pi i N+B M\}$, through which we factor. This proves that the Abel mapping is well-defined.

For $g=1$ (an elliptic Riemann surface) we have already considered the Abel mapping in Ch. I, $\S 3$ and have shown that it is an isomorphism of this Riemann surface onto the two-dimensional complex torus that is its Jacobi variety.

We apply the Abel mapping to solve the following problem. Let $f$ be a meromorphic function on a Riemann surface $\Gamma$. The number of its zeros on $\Gamma$ must be equal to the number of its poles (including multiplicities). The question arises: what sets of points $P_{1}, \ldots, P_{n}, Q_{1}, \ldots, Q_{n}$ can be zeros and poles of a meromorphic function on $\Gamma$ ? The answer is given by the following theorem

Abel's theorem. For points $P_{1}, \ldots, P_{n}, Q_{1}, \ldots, Q_{n}$ on a Riemann surface $\Gamma$ to be the zeros and poles of some meromorphic function it is necessary and sufficient that on the Jacobi variety $J(\Gamma)$

$$
\sum_{k=1}^{n} A\left(P_{k}\right)-\sum_{k=1}^{n} A\left(Q_{k}\right) \equiv 0
$$

(the symbol $\equiv$ here and later denotes congruence modulo the period lattice).

Proof. Suppose that a function $f$ on $\Gamma$ has zeros at $P_{1}, \ldots, P_{n}$ and poles at $Q_{1}, \ldots, Q_{n}$. We consider the meromorphic differential $\Omega=d \log f$. Now $\Omega$ 
has only simple poles with residue +1 at $P_{1}, \ldots, P_{n}$ and with residue -1 at $Q_{1}, \ldots, Q_{n}$. Therefore, $\Omega$ can be written in the form

$$
\Omega=\sum_{h=1}^{n} \omega_{P_{h} Q_{k}}+\sum_{j=1}^{g} c_{j} \omega_{j},
$$

where the $\omega_{p_{k}} Q_{k}$ are normalized differentials of the third kind, $\omega_{1}, \ldots, \omega_{g}$ are basis holomorphic differentials, and $c_{1}, \ldots, c_{g}$ are constants (see $\S 1$ above). Since $f$ is single-valued on $\Gamma$, the integral of $\Omega$ over any closed cycle must be an integer multiple of $2 \pi i$. In particular,

$$
\oint_{a_{k}} \Omega=2 \pi i n_{k}, \quad \oint_{b_{k}} \Omega=2 \pi i m_{k},
$$

where $n_{k}$ and $m_{k}$ are integers. Hence, bearing the normalization (2.1.20) in mind, we obtain

$$
2 \pi i n_{k}=\oint_{a_{k}} \Omega=2 \pi i c_{k}, \quad c_{k}=n_{k}
$$

Next, the formulae (2.1.22) for the $b$-periods of the $\omega_{P Q}$ give

$$
2 \pi i m_{k}=\underset{\substack{\dot{b} \\ b_{k}}}{6} \Omega=\sum_{j=1}^{n} \int_{\dot{Q}_{j}}^{P_{j}} \omega_{k}+\sum_{j=1}^{g} n_{j} B_{j k} .
$$

Hence we obtain

$$
\left[\sum_{j} A\left(P_{j}\right)-\sum_{j} A\left(Q_{j}\right)\right]_{k}=-\sum_{j} \int_{Q_{j}}^{P_{j}} \omega_{k}=-2 \pi i m_{k}+\sum n_{j} B_{j k} .
$$

The right-hand side of the equality is the $k$-th component of some vector of the period lattice, which means that (2.2.4) is valid. Carrying the argument out in the reverse order, we obtain a differential $\Omega$ with the required poles and with periods that are integer multiplies of $2 \pi i$. Then the function $f(P)=\exp \int_{P_{0}}^{P} \Omega$ is single-valued on $\Gamma$ and has the prescribed singularities. This proves the theorem.

\section{§3. Some remarks on divisors on a Riemann surface}

1. A divisor on a Riemann surface $\Gamma$ is a set of points of $\Gamma$ with multiplicities. It is convenient to write divisors as formal linear combinations of points of $\Gamma$ :

$$
D=\sum_{i=1}^{N} n_{i} P_{i} ; \quad n_{i}-\text { are integers. }
$$


For example, for any meromorphic function $f$ on $\Gamma$ we define its divisor $(f)$ of zeros $P_{1}, \ldots, P_{n}$ with multiplicities $p_{1}, \ldots, p_{n}$ and poles $Q_{1}, \ldots, Q_{m}$ with multiplicities $q_{1}, \ldots, q_{m}$ :

$$
(f)=p_{1} P_{1}+\ldots+p_{n} P_{n}-q_{1} Q_{1}-\ldots-q_{m} Q_{m} .
$$

Divisors form an Abelian group:

$$
D=\sum n_{i} P_{i}, \quad D^{\prime}=\sum n_{\boldsymbol{i}}^{\prime} P_{\boldsymbol{i}}^{\prime}, \quad D+D^{\prime}=\sum n_{i} P_{i}+\sum n_{i}^{\prime} P_{\boldsymbol{i}}^{\prime}
$$

(the zero is the "empty divisor").

2. The degree of a divisor $D=\sum n_{i} P_{i}$ is the number

$$
\operatorname{deg} D=\Sigma n_{i} \text {. }
$$

For example, the degree of the divisor of a meromorphic function is zero (the number of zeros is equal to the number of poles, including multiplicities). The degree is a linear function on the group of divisors: $\operatorname{deg}\left(D+D^{\prime}\right)=$ $=\operatorname{deg} D+\operatorname{deg} D^{\prime}$.

3. We say that two divisors $D$ and $D^{\prime}$ are linearly equivalent if their difference $D-D^{\prime}$ is the divisor of a meromorphic function. The divisors of meromorphic functions are linearly equivalent to zero (they are also called principal divisors). The degrees of two linearly equivalent divisors are equal.

Example. For any Abelian differential $\omega=f(z) d z$ on $\Gamma$ we consider its divisor of zeros and poles $(\omega)$. If $\eta=g(z) d z$ is another Abelian differential and $(\eta)$ its divisor, then $(\omega)$ and $(\eta)$ are linearly equivalent, since the quotient $\omega / \eta$ is a meromorphic function on $\Gamma$ ( $d z$ "cancels"). The equivalence class of divisors of all Abelian differentials is called the canonical class of $\Gamma$ and is denoted by $C$. The degree of the divisors of this class is $2 g-2$ (see [1]).

We extend the Abel mapping (2.2.1) linearly to the group of all divisors

$$
D=\sum_{i} n_{i} P_{i}, \quad A(D)=\sum_{i} n_{i} A\left(P_{i}\right)
$$

Now Abel's theorem can be reformulated in the following form: two divisors $D$ and $D^{\prime}$ are linearly equivalent if and only if the following two conditions hold:

1) $\operatorname{deg} D=\operatorname{deg} D^{\prime}$.

2) $A(D) \equiv A\left(D^{\prime}\right)$ on the Jacobi variety $J(\Gamma)$ (we recall that $\equiv$ means congruence modulo the period lattice).

4. A divisor $D=\sum n_{i} P_{i}$ is called positive (or effective) if all the multiplicities $n_{i}$ are positive. By definition, $D \geqslant \dot{D}^{\prime}$ for two divisors $D$ and $D^{\prime}$ if their difference $D-D^{\prime}$ is a positive divisor. We note a useful property of divisors of degree $\geqslant g$ : any such divisor is linearly equivalent to a positive divisor. This can be deduced, for example, from the results of the following section. 
With each divisor $D$ there is connected the linear space $L(D)$ of those meromorphic functions $f$ on $\Gamma$ for the divisors $(f)$ of which the following inequality holds:

$$
(f) \geqslant-D
$$

In particular, if $D=\sum n_{i} P_{i}$ is positive, then the space $L(D)$ consists of those meromorphic functions that can have poles only at the points $P_{i}$ of multiplicity not exceeding $n_{i}$. The dimension of the linear space $L(D)$ is denoted by $l(D)$. It is clear that if $D$ and $D^{\prime}$ are two linearly equivalent divisors, then the numbers $l(D)$ and $l\left(D^{\prime}\right)$ are the same (the spaces $L(D)$ and $L\left(D^{\prime}\right)$ are isomorphic).

For a positive divisor $D$ in general position the number $l(D)$ behaves as follows: 1) If $\operatorname{deg} D \leqslant g$, then the meromorphic functions with poles in $D$ are only constants, that is $l(D)=1$. In particular, if $D=n P$, where $P$ is a fixed point in general position on $\Gamma$ and $n$ is a positive integer, then $l(n P)=1$ for $n \leqslant g$. This means that there are, in general, no meromorphic functions (other than constants) with a single pole of multiplicity $\leqslant g$. Those points of a Riemann surface for which such meromorphic functions do exist are called Weierstrass points. For example, on a hyperelliptic

Riemann surface of the form $w^{2}=P_{2 g+2}(z)=\prod_{k=1}^{2 g+2}\left(z-z_{k}\right)$ each branch point $z=z_{i}(w=0)$ is a Weierstrass point, since the meromorphic function $f_{i}(z)=1 /\left(z-z_{i}\right)$ has a pole of order 2 there. For a hyperelliptic surface of genus $g$ given by a polynomial of odd degree $w^{2}=P_{2 g+1}(z)$ there are also $2 g+2$ Weierstrass points (one is the point as infinity).

If $\operatorname{deg} D \geqslant g$ for a divisor in general position, $l(D)$ is given by the formula

$$
l(D)=\operatorname{deg} D-g+1 .
$$

Such divisors are called non-special. For all the remaining divisors $D$ with $\operatorname{deg} D \geqslant 2$, which are called special, $l(D)>\operatorname{deg} D-g+1$. It turns out (see [4]), that the special divisors $D=P_{1}+\ldots+P_{N}, N=\operatorname{deg} D \geqslant g$ are precisely the critical points of the Abel mapping

$$
\left\{\begin{array}{l}
S^{N} \Gamma \stackrel{A}{\rightarrow} J(\Gamma), \\
A\left(P_{1}, \ldots, P_{N}\right)=A\left(P_{1}\right)+\ldots+A\left(P_{N}\right),
\end{array}\right.
$$

that is, those sets of points $\left(P_{1}, \ldots, P_{N}\right)$ at which the rank of the differential of the mapping (2.3.8) is less than $g$. Here $S^{N} \Gamma$ denotes the totality of unordered sets of points of $\Gamma$ (the " $N$-th symmetric power of $\Gamma$ ").

In the general case, information about $l(D)$ can be extracted from the Riemann-Roch theorem:

$$
l(D)=\operatorname{deg} D-g+1+l(C-D),
$$

where $C$ is the canonical class defined above. We do not discuss this theorem here (see [1]-[4]). 


\section{§4. The Jacobi inversion problem. Examples}

We saw above (see Ch. I, §3) that for $g=1$ the Abel mapping $A: \Gamma \rightarrow$ $\rightarrow J(\Gamma)$ is invertible and is an isomorphism. For larger genera $g>1$ the problem of inverting the Abel mapping can be stated as follows (the Jacobi inversion problem): for a given point $\zeta=\left(\zeta_{1}, \ldots, \zeta_{g}\right) \in J(\Gamma)$ to find $g$ points $P_{1}, \ldots, P_{g}$ of $\Gamma$ such that

$$
\sum_{k=1}^{g} \int_{P_{0}}^{P_{k}} \omega_{j} \equiv \zeta_{j} \quad(j=1, \ldots, g) .
$$

Here $\omega_{1}, \ldots, \omega_{g}$ is a canonical basis of holomorphic differentials on $\Gamma ; P_{0}$ is a fixed point of $\Gamma$. The system (2.4.1) must hold on the Jacobian $J(\Gamma)$ (the symbol $\equiv$, as usual, means congruence modulo the period lattice).

The unordered sets of $g$ points of $\Gamma$ form the $g$-th symmetric power $S^{g} \Gamma$ of $\Gamma$. In the language of the Abel mapping the problem (2.4.1) can be rewritten as follows: to invert the mapping

$$
A: S^{g} \Gamma \rightarrow J(\Gamma)
$$

where

$$
A\left(P_{1}, \ldots, P_{g}\right)=A\left(P_{1}\right)+\ldots+A\left(P_{g}\right) .
$$

We shall show that for almost any point $\zeta \in J(\Gamma)$ the set of points $\left(P_{1}, \ldots, P_{g}\right)=$ $=A^{-1}(\zeta)$ exists and is uniquely determined by the system (2.4.1) (without taking the order of these points into account).

To solve the Jacobi inversion problem we use the Riemann $\theta$-function $\theta(z) \equiv \theta(z \mid B)$ of $\Gamma$. Here $B=\left(B_{j k}\right)$ is the period matrix of holomorphic differentials on $\Gamma$ (see $\S 1$ above). Let $e=\left(e_{1}, \ldots, e_{g}\right) \in \mathbf{C}^{g}$ be a fixed vector. We consider the function

$$
F(P)=\theta(A(P)-e) .
$$

It is single-valued and analytic on the cut surface $\widetilde{\Gamma}$. We assume that $F(P)$ is not identically zero. This happens, for example, when $\theta(e) \neq 0$.

Lemma 2.4.1. If $F(P) \neq 0$, then $F(P)$ has $g$ zeros on $\widetilde{\Gamma}$ (including multiplicities).

Proof. To calculate the number of zeros we need to compute the logarithmic residue

$$
\frac{1}{2 \pi i} \int_{\widetilde{\mathrm{r}}} d \log F(P)
$$

(we assume that the zeros of $F(P)$ do not lie on $d \widetilde{\Gamma}$ ). For brevity we introduce the following notation (which is also useful later on): by $F^{+}$we denote the value that $F$ takes at a point on $d \widetilde{\Gamma}$ lying on the segment $a_{k}$ or $b_{k}$, and by $F^{-}$the value of $F$ at the corresponding point on $a_{k}^{-1}$ or $b_{k}^{-1}$ (see 
Fig. 2). The expressions $A^{+}$and $A^{-}$have an analogous meaning. In this notation the integral $(2.4 .5)$ can be rewritten in the form

(2.4.6) $\frac{1}{2 \pi i} \int_{\partial \widetilde{\Gamma}} d \log F(P)=\frac{1}{2 \pi i} \sum_{k=1}^{g}\left(\int_{a_{k}}+\int_{b_{k}}\right)\left[d \log F^{+}-d \log F^{-}\right]$.

We note that if $P$ is a point on $a_{k}$, then

$$
A_{j}^{-}(P)=A_{j}^{+}(P)+B_{j k},
$$

and if $P$ lies in $b_{k}$, then

$$
A_{j}^{+}(P)=A_{i}^{-}(P)+2 \pi i \delta_{j k}
$$

(see the proof of Lemma 2.1.1). From the transformation law of $\theta$-functions (formulae (1.1.4), (1.1.5)), we obtain on the cycle $a_{k}$

$$
\log F^{-}(P)=-\frac{1}{2} B_{k k}-A_{k}(P)+e_{k}+\log F_{j}^{+}(P) ;
$$

on $b_{k}$

$$
\log F^{+}=\log F^{-} .
$$

Since $d A_{k}(P)=\omega_{k}$, then we have on $a_{k}$

$$
d \log F^{-}(P)=d \log F^{+}(P)-\omega_{k} ;
$$

on $b_{k}$

$$
d \log F^{-}=d \log F^{+} \text {. }
$$

Thus, the sum (2.4.6) can be rewritten in the form

$$
\frac{1}{2 \pi i} \oint_{\partial \widetilde{\Gamma}} d \log F=\frac{1}{2 \pi i} \sum_{k} \int_{a_{k}} \omega_{k}=g,
$$

where we have normalized by $\oint_{i_{k}} \omega_{k}=2 \pi i$. This proves the lemma.

We now claim that the $g$ zeros of $F(P)$ also solve the Jacobi inversion problem for a suitable choice of the vector $e$.

Lemma 2.4.2. Let $F(P) \not \equiv 0$ and let $P_{1}, \ldots, P_{g}$ be its zeros on $\Gamma$. Then on $J(\Gamma)$

$$
A\left(P_{1}, \ldots, P_{g}\right) \equiv e-K,
$$

where $K=\left(K_{1}, \ldots, K_{g}\right)$ is the vector of Riemann constants, and

(2.4.14) $K_{j}=\frac{2 \pi i+B_{j j}}{2}-\frac{1}{2 \pi i} \sum_{l=j j} \int_{a_{l}}\left(\omega_{l}(P) \int_{P_{0}}^{P} \omega_{j}\right) \quad(j=1, \ldots, g)$.

Proof. We consider the following integral:

$$
\zeta_{j}=\frac{1}{2 \pi i} \oint_{i \widetilde{\Gamma}} A_{j}(P) d \log F(P), \quad(j=1, \ldots, g) .
$$


On the one hand, it is equal to the sum of the residues of the integrand, that is

$$
\zeta_{j}=A_{j}\left(P_{1}\right)+\ldots+A_{j}\left(P_{g}\right),
$$

where $P_{1}, \ldots, P_{g}$ are the zeros of $F(P)$. On the other hand, as in Lemma 2.4.1, we have (see (2.4.7)-(2.4.12))

$$
\begin{gathered}
\zeta_{j}=\frac{1}{2 \pi i} \sum_{k=1}^{g}\left(\int_{a_{k}}+\int_{b_{k}}\right)\left(A_{j}^{\dagger} d \log F^{+}-A_{j}^{-} d \log F^{-}\right)= \\
=\frac{1}{2 \pi i} \sum_{k=1}^{g} \int_{a_{k}}\left[A_{j}^{\dagger} d \log F^{+}-\left(A_{j}^{+}+B_{j k}\right)\left(d \log F^{+}-\omega_{k}\right)\right]+ \\
\quad+\frac{1}{2 \pi i} \sum_{k=1}^{g} \int_{b_{k}}\left[A_{j}^{+} d \log F^{+}-\left(A_{j}^{+}-2 \pi i \delta_{j k}\right) d \log F^{+}\right]= \\
=\frac{1}{2 \pi i} \sum_{k=1}^{g}\left(\int_{a_{k}} A_{j}^{+} \omega_{k}-B_{j k} \int_{\pi_{k}} d \log F^{+}+2 \pi i B_{j k}\right)+\int_{b_{i}} d \log F^{+} .
\end{gathered}
$$

$F$ takes the same values, at the ends of $a_{k}$, therefore

$$
\int_{a_{k}} d \log F^{+}=2 \pi i n_{k}
$$

where $n_{k}$ is an integer. Next, let $Q_{j}$ and $\widetilde{Q}_{j}$ be the beginning and end of $b_{j}$. Then

$$
\text { (2.4.18) } \begin{array}{r}
\int_{b_{j}} d \log F^{+}=\log F^{+}\left(\widetilde{Q}_{j}\right)-\log F^{+}\left(Q_{j}\right)+2 \pi i m_{j}= \\
=\log \theta\left(A\left(Q_{j}\right)+f_{j}-e\right)-\log \theta\left(A\left(Q_{j}\right)-e\right)+2 \pi i m_{j}= \\
=-\frac{1}{2} B_{j j}+e_{j}-A_{j}\left(Q_{j}\right)+2 \pi i m_{j},
\end{array}
$$

where $m_{j}$ is an integer and $f_{j}=\left(B_{j 1}, \ldots, B_{j g}\right)$ is a lattice vector.

The expression for $\zeta_{j}$ can now be rewritten as:

$$
\begin{aligned}
\zeta_{j}=e_{j}-\frac{1}{2} B_{j j}-A_{j}\left(Q_{j}\right)+\frac{1}{2 \pi i} \sum_{k} \int_{a_{k}} A_{j} \omega_{k}+ \\
+2 \pi i m_{j}+\sum_{k} B_{j k}\left(-n_{k}+1\right) .
\end{aligned}
$$

The last two terms in (2.4.19) can be dropped-they are coordinates of some lattice vector. To get rid of the term $A_{j}\left(Q_{j}\right)$ we transform the integral $\int_{a_{j}} A_{j} \omega_{j}$. We have $A_{j} \omega_{j}=\frac{1}{2} d\left(A_{j}^{2}\right)$, so that

$$
\int_{a_{j}} A_{j} \omega_{j}=\frac{1}{2}\left[A_{j}^{2}\left(Q_{j}\right)-A_{3}^{2}\left(R_{j}\right)\right]
$$


where $R_{j}$ is the beginning of $a_{j}$ and $Q_{j}$ is its end (which is also the beginning of $\left.b_{j}\right)$. Further, $A_{j}\left(Q_{j}\right)=A_{j}\left(R_{j}\right)+2 \pi i$. We obtain

$$
\int_{a_{j}} A_{j} \omega_{j}=\frac{1}{2} 2 \pi i\left(2 A_{j}\left(Q_{j}\right)-2 \pi i\right)
$$

hence

$$
-A_{j}\left(Q_{j}\right)+\frac{1}{2 \pi i} \sum_{k} \int_{a_{k}} A_{j} \omega_{k}=-\pi i+\frac{1}{2 \pi i} \sum_{k \neq j} \int_{a_{j}} A_{j} \omega_{k} .
$$

This proves the lemma.

Remark. The vector of Riemann constants $K$ is connected in a simple way with the canonical class $C$ of $\Gamma$ :

$$
2 K \equiv-A(C)
$$

Therefore, when the base point $P_{0}$ is skilfully chosen, the expression (2.4.14) simplifies (see [4]).

Thus, if the function $\theta(A(P)-e)$ is not identically zero on $\Gamma$, then its zeros solve the Jacobi inversion problem (2.4.1) for the vector $\zeta=e-K$. We state without proof the following criterion for $\theta(A(P)-e)$ to vanish identically (see [2], [4]).

Theorem 2.4.1. The function $\theta(A(P)-e)$ is identically zero on $\Gamma$ if and only if the point $e$ can be written in the form

$$
e \equiv A\left(Q_{1}\right)+\ldots+A\left(Q_{g}\right)+K
$$

where the divisor $D=Q_{1}+\ldots+Q_{g}$ is special.

We recall (see $\S 3$ above) that a divisor $D=Q_{1}+\ldots+Q_{g}$ is special if there exists a non-constant meromorphic function on $\Gamma$ whose poles can lie only at the points $Q_{1}, \ldots, Q_{g}$. If $D$ is in general position, there are no such functions.

Now we can prove the following important proposition.

Theorem 2.4.2. Let $\zeta=\left(\zeta_{1}, \ldots, \zeta_{g}\right)$ be a vector such that $F(P)=\theta(A(P)-$ $-\zeta-K)$ does not vanish identically on $\Gamma$. Then

a) $F(P)$ has $g$ zeros $P_{1}, \ldots, P_{g}$ on $\Gamma$, which give a solution of the Jacobi inversion problem

$$
\text { (2.4.22) } \quad A_{j}\left(P_{1}\right)+\ldots+A_{j}\left(P_{s}\right)=\sum_{k=1}^{g} \int_{P_{0}}^{P_{k}}\left(\omega_{j} \equiv \zeta_{j} \quad(j=1, \ldots g) .\right.
$$

b) The divisor $D=P_{1}+\ldots+P_{g}$ is non-special.

c) The points $P_{1}, \ldots, P_{g}$ are uniquely determined from the system (2.4.22) up to a permutation.

Proof. a) follows immediately from Lemmas 2.4.1 and 2.4.2. Further, if $D=P_{1},+\ldots+P_{g}$ were special, then it would follow from Theorem 2.4.1 
that $F(P) \equiv 0$, which is a contradiction. If $P_{1}^{\prime}, \ldots, P_{g}^{\prime}$ is another solution of (2.4.22), then the relation

$$
A\left(P_{1}\right)+\ldots+A\left(P_{g}\right) \equiv A\left(P_{1}^{\prime}\right)+\ldots+A\left(P_{g}^{\prime}\right)
$$

must hold on the Jacobi variety $J(\Gamma)$. By Abel's theorem this means that on $\Gamma$ there exists a meromorphic function with zeros at $P_{1}^{\prime}, \ldots, P_{g}^{\prime}$ and poles at $P_{1}, \ldots, P_{g}$. Since $D$ is non-special, such a function must be a constant, so that after renumbering $P_{i}=P_{i}^{\prime}$. This proves the theorem.

Later the following corollary will be useful.

Corollary. For a non-special divisor $D=P_{1}+\ldots+P_{g}$ of degree $g$ the function $F(P)=\theta(A(P)-A(D)-K)$ has exactly $g$ zeros $P=P_{1}, \ldots, P=P_{g}$ on $\Gamma$.

We also give for reference some information on the zeros of a $\theta$-function on $J(\Gamma)$ (a so-called $\theta$-divisor).

Theorem 2.4.3. The zeros of the $\theta$-function $\theta(e)=0$ admit a parametric representation in the form

$$
e \equiv A\left(P_{1}\right)+\ldots+A\left(P_{g-1}\right)+K
$$

where $P_{1}, \ldots, P_{g-1}$ is a set of $g-1$ arbitrary points of $\Gamma$.

Proof. Let $\theta(e)=0$. We set $F(P)=\theta(A(P)-e)$. Two cases are possible.

1) $F(P) \neq 0$ on $\Gamma$. Then, by Theorem 2.4 .2 ,

$$
e \equiv A\left(P_{1}\right)+\ldots+A\left(P_{g}\right)+K,
$$

where the set $P_{1}, \ldots, P_{g}$ is uniquely determined. Since $\theta(e)=0$, this set contains the point $P_{0}$ (the lower limit in the integrals); say, $P_{g}=P_{0}$. Then $A\left(P_{0}\right)=0$ and (2.4.24) implies that

$$
e \equiv A\left(P_{1}\right)+\ldots+A\left(P_{g-1}\right)+K .
$$

2) Let $F(P) \equiv 0$ on $\Gamma$. Then, by Theorem 2.4.1, we can represent $e$ in the form

$$
e \equiv A\left(Q_{1}\right)+\ldots+A\left(Q_{g}\right)+K
$$

where $D=Q_{1}+\ldots+Q_{g}$ is a special divisor. Because $D$ is special, there exists a meromorphic function $f$ on $\Gamma$ having poles at $Q_{1}, \ldots, Q_{g}$, such that $f\left(P_{0}\right)=0$. Let $D^{\prime}=P_{1}+\ldots+P_{g-1}+P_{0}$ be the divisor of the zeros of $f$. Then, by Abel's theorem, $A\left(D^{\prime}\right) \equiv A(D)$. Substituting $A\left(D^{\prime}\right)$ for $A(D)$ in (2.4.25) and again using the equality $A\left(P_{0}\right)=0$, we complete the proof of the theorem.

It has already been noted that the function $F(P)=\theta(A(P)-e$ ) (where $e=\zeta+K)$ does not vanish identically when $\theta(e) \neq 0$. The zeros of a $\theta$-function (the points of a $\theta$-divisor) form a subvariety of dimension $2 g-2$ (for $g \geqslant 3$ with singularities) in the $2 g$-dimensional torus $J(\Gamma)$. If the $\theta$-divisor can be dropped from $J(\Gamma)$, then we obtain a connected 
$2 g$-dimensional domain. So we find that for all the points of $J(\Gamma)$ the Jacobi inversion problem is soluble, and uniquely for almost all of the points.

Thus, the set of points $\left(P_{1}, \ldots, P_{g}\right)=A^{-1}(\xi)$ of $\Gamma$ (without counting the order of those points) is a single-valued function of $\zeta=\left(\zeta_{1}, \ldots, \zeta_{g}\right)$ of $J(\Gamma)$ (having "singularities" at the points of the $\theta$-divisor). To find an analytical expression for these functions, we take an arbitrary meromorphic function $f(P)$ on $\Gamma$. Then, the assignment of the quantities $\zeta_{1}, \ldots, \zeta_{g}$ uniquely determines the set of values

$$
f\left(P_{1}\right), \ldots, f\left(P_{g}\right), \quad\left(P_{1}, \ldots, P_{g}\right)=A^{-1}(\zeta) .
$$

Therefore, any symmetric function of these values is a single-valued meromorphic function of $\zeta=\left(\zeta_{1}, \ldots, \zeta_{g}\right) \in J(\Gamma)$, that is, an Abelian function on $J(\Gamma)$ (see Ch. I, §3). All these functions can be expressed in terms of a Riemann $\theta$-function. In an especially simple way one can express the following elementary symmetric functions ("Newton polynomials"):

$$
\sigma_{s}(\zeta)=\sum_{j=1}^{g} f^{s}\left(P_{j}\right) \quad(s=1,2, \ldots) .
$$

For them we obtain from Theorem 2.4.2 and the residue formula the following representation:

$$
\begin{aligned}
(2.4 .28) \quad \sigma_{s}(\zeta)=\frac{1}{2 \pi i} \oint_{\partial \widetilde{\Gamma}} f^{s}(P) d & \log \theta(A(P)-\zeta-K)- \\
& -\sum_{j\left(Q_{h}\right)=\infty} \operatorname{Res}_{P=Q_{h}} f^{s}(P) d \log \theta(A(P)-\zeta-K)
\end{aligned}
$$

(the second term on the right-hand side is the sum of the residues of the integrand at all the poles of $f(P)$ ). As in the proof of Lemmas 2.4.1 and 2.4 .2 , one can transform the first term in (2.4.28) by using (2.4.11) and (2.4.12). Then (2.4.28) can be rewritten as follows:

$$
\begin{aligned}
\sigma_{s}(\zeta)=\frac{1}{2 \pi i} \sum_{k} \int_{a_{k}} f^{s}(P) & \left(\omega_{k}-\right. \\
& -\sum_{f\left(Q_{k}\right)=\infty} \operatorname{Res}_{P=Q_{k}} f^{s}(P) d \log \theta(A(P)-\zeta-K) .
\end{aligned}
$$

Here the first term is a constant independent of $\zeta$. We consider the computation of the second term (of the sum of the residues) in two examples.

Example $1 . \Gamma$ is a hyperelliptic Riemann surface of genus $g$, given by the equation

$$
w^{2}=P_{2 g i-1}(z),
$$

where $P_{2 g+1}(z)$ is the polynomial of degree $2 g+1$ without multiple roots. We consider the following function on $\Gamma: f(w, z)=z$ (the projection on the 
$z$-plane). This function on $\Gamma$ has a single two-fold pole at the branch point (that is, the Weierstrass point) $z=\infty$. We obtain the analytic expression for the functions $\sigma_{1}$ and $\sigma_{2}$ constructed by (2.4.27). In other words, if $P_{1}=\left(w_{1}, z_{1}\right), \ldots, P_{g}=\left(w_{g}, z_{g}\right)$ is the solution of the inversion problem $A\left(P_{1}\right)+\ldots+A\left(P_{g}\right)=\zeta$, then

$$
\begin{aligned}
& \sigma_{1}(\zeta)=z_{1}+\ldots+z_{g} \\
& \sigma_{2}(\zeta)=z_{1}^{2}+\ldots+z_{g}^{2} .
\end{aligned}
$$

For the basis point $P_{0}$ (the lower limit of the integrals in the Abel mapping) we take $z=\infty$. By (2.4.29), the functions $\sigma_{1}$ and $\sigma_{2}$ have the form

$$
\begin{aligned}
& \sigma_{1}(\zeta)=c_{1}-\operatorname{Res}_{z=\infty} z d \log \theta(A(P)-\zeta-K), \\
& \sigma_{2}(\zeta)=c_{2}-\operatorname{Res}_{z=\infty} z^{2} d \log \theta(A(P)-\zeta-K),
\end{aligned}
$$

where $c_{1}$ and $c_{2}$ are constants depending only on the Riemann surface and on the choice of the basis of cycles on it. Let us compute the residue in (2.4.33). We take $\tau=1 / z$ as a local parameter in a neighbourhood of $z=\infty$. By the definition of the Abel mapping we immediately obtain

$$
\begin{aligned}
d \log \theta(\boldsymbol{A}(P)-\zeta-K)=\sum_{i=1}^{\xi} & {[\log \theta(A(P)-\zeta-K)]_{i} \omega_{i}(P)=} \\
= & \sum_{i=1}^{\xi}[\log \theta(A(P)-\zeta-K)]_{i} f_{i}(\tau) d \tau,
\end{aligned}
$$

where $[\ldots]_{i}$ denotes the partial derivative with respect to the $i$-th variable, and $\omega_{i}=f_{i}(\tau) d \tau(i=1, \ldots, g)$ is the canonical basis of holomorphic differentials on $\Gamma$. We expand the vector-valued function $A(P)$ in a series in powers of $\tau$ (for $P \rightarrow \infty$ ). By the choice of the basis point $P_{0}=\infty$, this expansion has the form

$$
A(P)=\tau U+\tau^{2} V+O\left(\tau^{3}\right),
$$

where $U=\left(U_{1}, \ldots, U_{g}\right)$ and $V=\left(V_{1}, \ldots, V_{g}\right)$ are the vectors of the form

$$
\begin{gathered}
U_{k}=\left.\frac{d A_{k}(P)}{d \tau}\right|_{P=\infty}=f_{k}(0), \\
V_{k}=\left.\frac{1}{2} \frac{d^{2} A_{k}(P)}{d \tau^{2}}\right|_{P=\infty}=\frac{1}{2} f_{k}^{\prime}(0)
\end{gathered}
$$

(we recall that $\tau=0$ corresponds to the point $\infty$ on $\Gamma$ ).

Lemma 2.4.3. a) $U$ is the vector of b-periods of a normalized differential of the second kind with a single double pole at $z=\infty$ and principal part $d \tau / \tau^{2}$.

b) $V$ is the null vector. 
Proof. a) follows immediately from Lemma $2.1 .2((2.1 .21)$ for $n=1)$. From the same lemma we see that $V$ (up to an unessential factor) is the period vector of a differential of the second kind with a pole of the third order at $\infty$. But this differential is exact-it is equal to $d z=2 d \tau / \tau^{3}$, therefore, it has zero periods over all the cycles. This proves the lemma.

Thus, the expansion (2.4.36) has the form

$$
A(P)=\tau U+O\left(\tau^{3}\right) .
$$

Therefore, in a neighbourhood of $\infty$

$$
\log \theta(A(P)-\zeta-K)=\log \theta(\zeta+K)-\tau \sum_{j} U_{j}[\log \theta(\zeta+K)]_{j}
$$

(we have used the fact that the $\theta$-function is even),

$$
f_{1}(\tau)=U_{i}+O\left(\tau^{2}\right)
$$

Hence, for $\sigma_{1}(\zeta)$ we obtain the expression

$$
\sigma_{1}(\zeta)=\sum_{i, j} U_{i} U_{j}[\log \theta(\zeta+K)]_{i j}+c_{1} .
$$

We introduce the operator $\partial / \partial x=\Sigma U_{i} \partial / \partial \zeta_{i}$ of the derivative along $U$. Then (2.4.40) can be rewritten in the form

$$
\sigma_{1}(\zeta)=\frac{\partial^{2}}{\partial x^{2}} \log \theta(\zeta+h)+c_{1} .
$$

An analogous (but longer) calculation of the residue in (2.4.34) leads to the following expression for $\sigma_{2}$ :

$$
\sigma_{2}(\zeta)=\left(-\frac{4}{3} \frac{1}{\partial x \partial t}-\frac{1}{6} \frac{\partial^{t}}{\partial x^{4}}\right) \log 0(\zeta+K)+c_{2} .
$$

Here $\partial / \partial t=\Sigma W_{i} \partial / \partial \xi_{i}$ is the differentiation operator along the vector $W=\left(W_{1}, \ldots, W_{g}\right)$, where

$$
W_{k}=\frac{1}{3} f_{h}^{\prime \prime}(0) \text {. }
$$

It follows from Lemma 2.1.2 that $W$ is the vector of $b$-periods of a normalized differential of the second kind with a single pole of the fourth order at $\infty$ with principal part $3 d \tau / \tau^{4}$.

The formulae (2.4.41) and (2.4.42) completely solve the Jacobi inversion problem for Riemann surfaces of genus 2 (all these surfaces are hyperelliptic).

We shall show in Chapter III that the function $u=2 \sigma_{1}$ is a solution of the Korteweg-de Vries equation

$$
\frac{\partial u}{\partial t}=\frac{1}{4}\left(6 u \frac{\partial u}{\partial x}+\frac{\partial^{3} u}{\partial x^{3}}\right) .
$$

Using this equation it is easy to reduce (2.4.42) to the form

$$
\sigma_{2}(\zeta)=-2 \sigma_{1}^{2}(\zeta)-\frac{1}{2} \frac{\partial^{2} \sigma_{1}(\zeta)}{\partial x^{2}}+\tilde{c_{2}}
$$

which only includes differentiation operators in the $x$-direction. 
Remark. We recall that the basis holomorphic differentials $\omega_{1}, \ldots, \omega_{g}$ on the Riemann surface (2.4.30) have the form

$$
\omega_{k}=\frac{c_{k 1} z^{g-1}+c_{k 2^{z}} g^{-2}+\ldots+c_{k g}}{\sqrt{P_{2 g+1}(z)}} d z .
$$

Here the matrix $c_{j k}$ has the form $\left(c_{j k}\right)=2 \pi i\left(A_{j k}\right)^{-1}$, where

$$
A_{j k}=\oint_{a_{j}} \frac{z^{k-1} d z}{\sqrt{P_{a} g_{+1}(z)}} \quad(j, k=1, \ldots, g) .
$$

Therefore, the vector $\left(U_{1}, \ldots, U_{g}\right)$ (see above) has the following coordinates:

$$
U_{k}=-2 c_{k 1} \text {. }
$$

We do not derive the analogous expression for $W$.

Example 2. Now let $\Gamma$ be the hyperelliptic surface of genus $g$ given by the equation

$$
w^{2}=P_{2 g+2}(z) .
$$

We consider again the function $f(w, z)=z$ and the function $\sigma_{1}(\zeta)$ (the sum of the projections on the $z$-plane) constructed from it. In this case $\Gamma$ has two points at infinity: $P_{+}=(\infty,+)$ and $P_{-}=(\infty,-)$ at each of which $z$ has a simple pole. For $\sigma_{1}(2.4 .49)$ can be written in the form

$$
\sigma_{1}(\zeta)=c_{1}-\left[\operatorname{Res}_{\boldsymbol{P}=P_{+}}+\operatorname{Res}_{\boldsymbol{P}=P_{-}}\right] z d \log \theta(A(P)-\zeta-K)
$$

We can take $\tau=1 / z$ as a local parameter at both points $P_{+}$and $P_{-}$. Here the holomorphic differentials $\omega_{1}, \ldots, \omega_{g}$ have the form

$$
\omega_{k}=\frac{c_{k 1} z^{g-1}+\ldots+c_{k g}}{\sqrt{\overline{P_{2 g+2}(z)}}} d z .
$$

Let $\omega_{k}=f_{k}^{+}(\tau) d \tau$ in a neighbourhood of $P_{+}$(which corresponds to the value $\tau=0$ ) and let $\omega_{k}=f_{\bar{k}}(\tau) d \tau$ in a neighbourhood of $P_{-}$(which corresponds to the value $\tau=0$ ). From (2.4.49) it follows that

$$
f_{\bar{k}}^{-}(0)=-f_{k}^{+}(0) \text {. }
$$

We denote $f_{k}^{+}(0)$ by $U_{k}(k=1, \ldots, g)$.

The calculation of the residues in (2.4.48), similar to the one considered in Example 1, yields

$$
\sigma_{1}(\zeta)=c_{1}+\sum U_{i}\left[\log \frac{\theta\left(\zeta+K-A\left(P_{+}\right)\right)}{\theta\left(\zeta+K-A\left(P_{-}\right)\right)}\right]_{i} .
$$

By introducing the differentiation operator $\partial / \partial x=\sum U_{i} \partial / \partial \zeta_{i}$ in the direction of the vector $U=\left(U_{1}, \ldots, U_{g}\right)$, we finally obtain

$$
\sigma_{1}(\zeta)=\frac{\partial}{\partial x} \log \frac{\theta\left(\zeta+K-A\left(P_{+}\right)\right)}{\theta\left(\zeta+K-A\left(P_{-}\right)\right)}+c_{1},
$$


where $c_{1}$ is a constant. The components of $U$ are the periods of a normalized differential of the second kind with a double pole at $P_{+}$-this follows from Lemma 2.1.2.

The method explained above for solving the Jacobi inversion problem is due to Riemann. We should also mention here the approach of Weierstrass, who studied the system of differential equations resulting from (2.4.1) by differentiation. We only analyse the case $g=2$, where $\Gamma$ is given by the equation

$$
w^{2}=P_{5}(z)
$$

As a basis (non-normalized) of holomorphic differentials we take the differentials

$$
\omega_{1}=\frac{d z}{\sqrt{P_{5}(z)}}, \quad \omega_{2}=\frac{z d z}{\sqrt{P_{5}(z)}} .
$$

We consider two systems of differential equations:

$$
\begin{gathered}
\frac{d z_{1}}{d x}=\frac{\sqrt{P_{5}\left(z_{1}\right)}}{z_{1}-z_{2}}, \quad \frac{d z_{2}}{d x}=\frac{\sqrt{P_{5}\left(z_{2}\right)}}{z_{2}-z_{1}} ; \\
\frac{d z_{1}}{d t}=\frac{z_{2} \sqrt{P_{5}\left(z_{1}\right)}}{z_{1}-z_{2}}, \quad \frac{d z_{2}}{d t}=\frac{z_{1} \sqrt{P_{5}\left(z_{2}\right)}}{z_{2}-z_{1}} .
\end{gathered}
$$

Each of these systems determines the law of motion for a pair of points

$$
P_{1}=\left(z_{1}, \sqrt{P_{5}\left(z_{1}\right)}\right), \quad P_{2}=\left(z_{2}, \sqrt{P_{5}\left(z_{2}\right)}\right)
$$

on $\Gamma$. The following simple lemma holds.

Lemma 2.4.4. For the Abel mapping $S^{2} \Gamma \stackrel{A}{\rightarrow} J(\Gamma)$,

$$
\left(P_{1}=\left(z_{1}, \sqrt{P_{5}\left(z_{1}\right)}\right), \quad P_{2}=\left(z_{2}, \sqrt{P_{5}\left(z_{2}\right)}\right)\right) \mapsto\left(\zeta_{1}, \zeta_{2}\right),
$$

where

$$
\left\{\begin{array}{l}
\zeta_{1}=\int_{P_{0}}^{P_{1}} \frac{d z}{\sqrt{P_{5}(z)}}+\int_{P_{0}}^{P_{2}} \frac{d z}{\sqrt{P_{5}(z)}}, \\
\zeta_{2}=\int_{P_{0}}^{P_{1}} \frac{z d z}{\sqrt{P_{5}(z)}}+\int_{P_{0}}^{P_{2}} \frac{z d z}{\sqrt{P_{5}(z)}}
\end{array}\right.
$$

( $P_{0}$ is a fixed point), both systems (2.4.55) and (2.4.56) go over to systems with constant coefficients

$$
\begin{gathered}
\frac{d \zeta_{1}}{d x}=0, \quad \frac{d \zeta_{2}}{d x}=1 \\
\frac{d \zeta_{1}}{d t}=-1, \quad \frac{d \zeta_{2}}{d t}=0 .
\end{gathered}
$$

Thus, the Abel mapping (2.4.57) is simply a change of variables, which integrates (2.4.55) and (2.4.56). 
Proof. We have

$$
\begin{aligned}
& \frac{d \zeta_{1}}{d x}=\frac{d z_{1} / d x}{\sqrt{P_{5}\left(z_{1}\right)}}+\frac{d z_{2} / d x}{\sqrt{P_{5}\left(z_{2}\right)}}=\frac{1}{z_{1}-z_{2}}+\frac{1}{z_{2}-z_{1}}=0 \\
& \frac{d \zeta_{2}}{d x}=\frac{z_{1}}{z_{1}-z_{2}}+\frac{z_{2}}{z_{2}-z_{1}}=1 \\
& \frac{d \zeta_{1}}{d t}=\frac{z_{2}}{z_{1}-z_{2}}+\frac{z_{1}}{z_{2}-z_{1}}=-1, \\
& \frac{d \zeta_{1}}{d t}=\frac{z_{1} z_{2}}{z_{1}-z_{2}}+\frac{z_{2} z_{1}}{z_{2}-z_{1}}=0 .
\end{aligned}
$$

This proves the lemma.

By (2.4.58) and (2.4.59) the variables $x$ and $t$ are (complex) coordinates on the Jacobi torus $T^{4}=J(\Gamma)$. Therefore, the integration of the systems (2.4.55) and (2.4.56) $P_{1}=P_{1}(x, t), P_{2}=P_{2}(x, t)$ solves the Jacobi inversion problem.

We mention that these systems can easily be integrated by quadratures (for explicit formulae see the survey [17]). The expression of their solutions in terms of the $\theta$-function of $\Gamma$ can be obtained from the formulae of Example 1 (see above).

\section{ChAPTER III}

THE BAKER-AKHIEZER FUNCTION. APPLICATIONS TO NON-LINEAR EQUATIONS

$\S 1$. The Baker-Akhiezer one-point function. The Kadomtsev-Petviashvili equation and equations associated with it.

Let $\Gamma$ be a Riemann surface of genus $g$. We fix on $\Gamma$ a point $Q$ and a local parameter $z=z(P)$ in a neighbourhood of it (such that $z(Q)=0$ ). It is convenient to introduce the inverse $k=1 / z, k(Q)=\infty$. Suppose, further, that an arbitrary polynomial $q(k)$ is given.

Definition 3.1.1. Let $D=P_{1}+\ldots+P_{g}$ be a positive divisor of degree $g$ on $\Gamma$. $A$ Baker-Akhiezer function on $\Gamma$ corresponding to $Q$, to the local parameter $z=1 / k$ at $Q$, to the polynomial $q(k)$, and to the divisor $D$ is a function $\psi(P)$ such that:

a) $\psi(P)$ is meromorphic everywhere on $\Gamma$, except at $P=Q$, and has on $1 \backslash Q$ poles only at the points $P_{1}, \ldots, P_{g}$ of $D$ (more precisely, the divisor of the poles is $\psi \mid \Gamma \backslash Q \geqslant-D$; see $\mathrm{Ch}$. II, $\S 3)$;

b) the product $\psi(P) \exp [-q(k)]$ is analytic in a neighbourhood of $P=Q$.

Instead of b) we also say that $\psi(P)$ has at $P=Q$ an essential singularity of the form $\psi(P) \sim c \cdot \exp q(k)$ ( $c$ is a constant). For a given divisor $D$ such Baker-Akhiezer functions form a linear space (we fix the point $Q$, the local parameter $1 / k$, and the polynomial $q(k)$ ), which we denote by $\Lambda(D)$ (by analogy with the space $L(D)$ considered in $\mathrm{Ch}$. II, §3). 
Theorem 3.1.1. Let $D=P_{1}+\ldots+P_{g}$ be a non-special divisor of degree $g$. Then the space $\Lambda(D)$ for a polynomial $q$ in general position is one-dimensional.

In other words, for a non-special divisor $D$ and a general polynomial $q(k)$, the conditions of Definition 3.1.1 uniquely determine the Baker-Akhiezer functions, up to multiplication by a constant.

Proof. a) Uniqueness. The Baker-Akhiezer function has $g$ zeros on $\Gamma \backslash \infty \boldsymbol{g}$, and for a general polynomial $q(k)$ the divisor of zeros $\widetilde{D}$ is also non-special. Suppose now that $\psi(P)$ and $\widetilde{\psi}(P)$ are two Baker-Akhiezer functions corresponding to the same divisor $D$. Then their quotient $\psi(P) / \widetilde{\psi}(P)$ is a meromorphic function on $\Gamma$ (the essential singularity cancels) with poles at the points of $\widetilde{D}$. Since this divisor is non-special such a function is necessarily a constant.

b) Existence. Suppose that $\Omega$ is a differential of the second kind on $\Gamma$ with principal part at $Q$ of the form $d q(k)$, normalized by the conditions

$$
\oint_{a_{j}} \Omega=0 \quad(j=1, \ldots g) .
$$

Let $U=\left(U_{1}, \ldots, U_{g}\right)$ be its vector of $b$-periods:

$$
U_{k}=\oint_{b_{k}} \Omega
$$

We fix an arbitrary point $P_{0} \neq Q$ on $\Gamma$, take the corresponding Abel mapping $A(P)$, and construct the function

$$
\psi(P)=\exp \left(\int_{P_{0}}^{P} \Omega\right) \frac{\theta(A(P)-A(D)+U-K)}{\theta(A(P)-A(D)-K)},
$$

where $D$ is the given non-special divisor. The path of integration in the integral $\int_{P_{0}}^{P} \Omega$ and in the Abel mapping $A(P)=\left(\int_{P_{0}}^{P} \omega_{1}, \ldots, \int_{P_{0}}^{P} \omega_{g}\right)$ is chosen to be the same.

We claim that (3.1.3) is the required Baker-Akhiezer function. First, we verify that it is unique on $\Gamma$. If we choose another path of integration from $P_{0}$ to $P$, then we have to add to $\int_{P_{0}}^{P} \Omega$ a term of the form $\oint_{\gamma} \Omega$, where $\gamma$ is a closed contour (cycle). Similarly, the vector $\left(\oint_{\gamma} \omega_{1}, \ldots, \oint_{\gamma} \omega_{g}\right)$ is added to $A(P)$. We split $\gamma$ over the basis cycles:

$$
\gamma=\sum_{k=1}^{g} n_{k} a_{k}+\sum_{j=1}^{g} m_{j} b_{j}
$$


where $n_{k}$ and $m_{j}$ are integers. Then on changing the path of integration we have

$$
\begin{gathered}
\int_{\dot{P}_{0}}^{P} \Omega \rightarrow \int_{P_{0}}^{\mathrm{P}} \Omega+\sum m_{\jmath} U_{\jmath}=\int_{P_{0}}^{P} \Omega+\langle M, U\rangle, \\
A(P) \mapsto A(P)+2 \pi i N+B M .
\end{gathered}
$$

Here $M=\left(m_{1}, \ldots, m_{g}\right)$ and $N=\left(n_{1}, \ldots, n_{g}\right)$ are integral vectors. Under such a transformation, by (1.1.6), the quotient of the $\theta$-functions is multiplied by

$$
\frac{\exp \left[-\frac{1}{2}\langle B M, M\rangle-\langle M, A(P)-A(D)+U-K\rangle\right]}{\exp \left[-\frac{1}{2}\langle B M, M\rangle-\langle M, A(P)-A(D)-K\rangle\right]}=\exp (-\langle M, U\rangle),
$$

and the exponentional term acquires the inverse factor $\exp \langle M, U\rangle$. This proves the uniqueness.

Next, because the divisor $D=P_{1}+\ldots+P_{g}$ is non-special, the poles of (3.1.3) (arising from the zeros of the denominator) lie exactly at the points $P_{1}, \ldots, P_{g}$-see the Corollary to Theorem 2.4.2. Moreover, the function (3.1.3) has an essential singularity of the kind needed, by the choice of: $\Omega=d q(k)+\ldots, \int \Omega=q(k)+\ldots$ in a neighbourhood of $Q$ (the dots denote regular terms). This proves the theorem.

We analyse in more detail the Baker-Akhiezer function constructed from the polynomial

$$
q(k)=k x+k^{2} y+k^{3} t,
$$

where $x, y$, and $t$ are the parameters. We denote this function corresponding to some non-special divisor $D$ of degree $g$ by $\psi(x, y, t ; P)$. Then $\psi(x, y, t ; P)$ can be normalized so that in a neighbourhood of $Q$ its expansion takes the form

$$
\psi(x, y, t ; P)=e^{k x+k^{2} y+k^{3} t}\left(1+\frac{\xi_{1}}{k}+\frac{\xi_{2}}{k^{\mathbf{s}}}+\ldots\right) .
$$

Here the coefficients $\xi_{1}, \xi_{2}, \ldots$ are functions of $x, y$, and $t$ (which we shall calculate below).

Let us leave Riemann surfaces for a while and regard the expansion (3.1.8) as formal (without being interested in its convergence). We have a simple but important lemma.

Lemma 3.1.1. For a function $\psi$ of the form (3.1.8) the formal equalities

$$
\text { (3.1.10) }\left[-\frac{\partial}{\partial t}+\frac{\partial^{3}}{\partial x^{3}}+\frac{3}{2} u \frac{\partial}{\partial x}+w\right] \psi=O\left(\frac{1}{k}\right) e^{h x+k^{2} y+k^{3} t},
$$


hold, where the functions $u$ and $w$ can be found from the condition for the vanishing of the coefficients of $k^{n} e^{k x+k^{2} y+k^{3} t}$ for $n=3,2,1,0$. These functions have the form

$$
\begin{gathered}
u=-2 \frac{\partial \xi_{1}}{\partial x}, \\
w=3 \xi_{1} \frac{\partial \xi_{1}}{\partial x}+3 \frac{\partial^{2} \xi_{1}}{\partial x^{2}}-3 \frac{\partial \xi_{2}}{\partial x} .
\end{gathered}
$$

The proof is by direct calculation.

We denote by $L$ and $A$ the ordinary differential operators in $x$ :

$$
\begin{gathered}
L=\frac{\partial^{2}}{\partial x^{2}}+u, \\
A=\frac{\partial^{3}}{\partial x^{3}}+\frac{3}{2} u \frac{\partial}{\partial x}+-w .
\end{gathered}
$$

Theorem 3.1.2. Let $\psi=\psi(x, y, t ; P)$ be a Baker-Akhiezer function constructed from the polynomial $q(k)=k x+k^{2} y+k^{3} t$ and corresponding to some non-special divisor $D$ of degree $g$. Then $\psi$ is a solution of the system of equations

$$
\begin{aligned}
& \frac{\partial \psi}{\partial y}=L \psi, \\
& \frac{\partial \psi}{\partial t}=A \psi
\end{aligned}
$$

where the operators $L$ and $A$ are given by (3.1.13) and (3.1.14).

Proof. The functions $\varphi_{1}=\left(-\frac{\partial}{\partial y}+L\right) \psi$ and $\varphi_{2}=\left(-\frac{\partial}{\partial t}+A\right) \psi$ satisfy all the conditions of the definition of a Baker-Akhiezer function. But it follows from Lemma 3.1.1 that the values of the products $\varphi_{1} \exp (-k x-$ $\left.-k^{2} y-k^{3} t\right)$ and $\varphi_{2} \exp \left(-k x-k^{2} y-k^{3} t\right)$ at $Q$ are zero. From uniqueness of the Baker-Akhiezer function (Theorem 3.1.1) it then follows that $\varphi_{1}=\varphi_{2} \equiv 0$ on $\Gamma$.

Corollary. The functions $u$ and $w$ of the form (3.1.11), (3.1.12) give a solution to the system of non-linear equations

$$
\left\{\begin{array}{l}
\frac{3}{2} u_{y}+\frac{3}{2} u_{x x}-2 w_{x}=0, \\
w_{y}-u_{t}+u_{x x x}+\frac{3}{2} u u_{x}-w_{x x}=0 .
\end{array}\right.
$$

Eliminating $w$ from this system we arrive at the famous KadomtsevPetviashvili (KP) equation

$$
\frac{3}{4} u_{y y}=\frac{\partial}{\partial x}\left[u_{t}-\frac{1}{4}\left(6 u u_{x}+u_{x x x}\right)\right] .
$$


Proof of the Corollary. The condition of compatibility of the equations $\partial \psi / \partial y=L \psi$ and $\partial \psi / \partial t=A \psi$ has the form

$$
\left[-\frac{\partial}{\partial y}+L,-\frac{\partial}{\partial t}+A\right]=0 \text {. }
$$

(By $[\ldots, \ldots]$ we denote the commutator of the operators). By computing this commutator we obtain (3.1.17).

Thus, for each Riemann surface $\Gamma$ of genus $g$, each point $Q$ on it, and each local parameter $k^{-1}$ we can construct in a neighbourhood of $Q$ a family of solutions of the KP equation, parametrized by the non-special divisors of degree $g$ on $\Gamma$ (the points in general position of the Jacobi variety $J(\Gamma)$ ).

The change of the local parameter

$$
k \mapsto \lambda k+a+\frac{b}{k}+O\left(\frac{1}{k^{2}}\right)
$$

(where $\lambda, a, b$ are arbitrary complex numbers and $\lambda \neq 0$ ) leads to another family of solutions of the same KP equation. It is easy to verify that these other solutions are obtained by means of the following transformations, which preserve the form of the KP equation:

$$
\left\{\begin{aligned}
x & \mapsto \lambda x+2 \lambda a y+\left(3 \lambda a^{2}+3 \lambda^{2} b\right) t, \\
y & \rightarrow \lambda^{2} y+3 \lambda^{2} a t, \\
t & \mapsto \lambda^{3} t, \\
u & \mapsto \frac{1}{\lambda^{2}} u-\frac{2 b}{\lambda} .
\end{aligned}\right.
$$

Of course, the fact that the KP equation admits the group of transformations of the form (3.1.21) is trivial to verify (without resort to the theory of $\theta$-functions).

We now express the thus constructed solutions in terms of the $\theta$-function of $\Gamma$. To do this we make use of the formula (3.1.3) for the Baker-Akhiezer function, which in our case is as follows:

$$
\begin{aligned}
\psi(x, y, t ; P)=\exp \left(x \int_{P_{0}}^{P} \Omega^{(1)}+y \int_{P_{0}}^{P} \Omega^{(2)}+t \int_{P_{0}}^{P} \Omega^{(3)}\right) \times \\
\quad \times \frac{\theta(A(P)-A(D)+x U+y V+t W-K)}{\theta(A(P)-A(D)-K)} .
\end{aligned}
$$

Here $\Omega^{(1)}, \Omega^{(2)}$, and $\Omega^{(3)}$ are normalized differentials of the second kind, with poles only at $Q$ and with principal parts also at $Q$ of the form

(3 $\left.1 \begin{array}{ll}2 & 23\end{array}\right) \quad \Omega^{(1)}=d k+\ldots, \quad \Omega^{(2)}=d\left(k^{2}\right)+\ldots, \quad \Omega^{(3)}=d\left(k^{3}\right)+\ldots$

(the dots denote the regular terms; $k^{-1}$ is the local parameter); $U, V$, and $W$ are their vectors of $b$-periods:

$$
U_{i}=\oint_{b_{i}} \Omega^{(1)}, \quad V_{i}=\oint_{b_{i}} \Omega^{(2)}, \quad W_{i}=\oint_{b_{i}} \Omega^{(3)} \quad(i=1, \ldots, g) .
$$


Theorem 3.1.3. The thus constructed solutions of the KP equation have the form

$$
u(x, y, t)=2 \frac{\partial^{2}}{\partial x^{2}} \log \theta\left(x U+y V+t W+z_{0}\right)+c,
$$

where $\theta=\theta(z)$ is the $\theta$-function on $\Gamma$, the vectors $U, V$, and $W$ are determined by (3.1.24), $z_{0}=-A(D)-K$ is an arbitrary vector, and $c$ is a constant.

Proof. By (3.1.11), it suffices to find the coefficient $\xi_{1}$ of the expansion (3.1.8). We note that the expansion of the logarithm of the Baker-Akhiezer function (not necessarily normalized) has the form

$$
\log y=h x+k^{2} y+k^{3} t+-\xi_{0}+\frac{\xi_{1}+c x+a y+b t}{k}+\ldots
$$

( $\xi_{0}$ is some function of $x, y, t ; a, b$, and $c$ are constants). Consequently, $\xi_{1}+c x+a y+b t$ is the coefficient of $1 / k$ in the expansion of the function

$$
\varphi\left(P^{\prime}\right)=\log \frac{\theta\left(A\left(P^{\prime}\right)-A(D)-K+x U+y V+(W)\right.}{\theta\left(A\left(I^{\prime}\right)-A(D)-K\right)}
$$

in a neighbourhood of $P=Q$. Next, we recall that it follows from (2.1.21) that the vector-valued function $A(P)$ as $P \rightarrow Q$ has the expansion:

$$
A(P)=A(Q)-\frac{1}{k} U+O\left(\frac{1}{k^{2}}\right) .
$$

We choose $Q$ as the initial point of the Abel mapping. Then $A(Q)=0$, and the required coefficient $\xi_{1}$ has the form

$$
\text { (3.1.28) } \quad \xi_{1}+c x+a y+-b t=-\frac{\partial}{\partial x} \log 0(x U+y \Gamma+t W-A(D)-K)+\ldots
$$

where the dots denote terms independent of $x, y, t$. From the formula $u=-2\left(\partial \xi_{1} / \partial x\right)$ we obtain the proof of the theorem.

(3.1.25) gives additional information about the properties of the solutions of the KP equation: $u(x, y, t)$ is a conditionally periodic (generally speaking, meromorphic) function of the variables $x, y$, and $t$. For the second logarithmic derivative $\left(\partial^{2} / \partial x^{2}\right) \log \theta(z)$ ( $z$ is a point of the Jacobian $J(\Gamma)$ ) is a meromorphic function on the torus $J(\Gamma)$ (an Abelian function). To obtain the solution $u(x, y, t)$ we have to restrict this function to the linear $(x-y-t)$-winding, spanned by the vectors $U, V$, and $W$. We do not discuss here the problem of isolating the real and the bounded solutions among those obtained.

If for $U$ we have the commensurability relation

$$
\text { (3.1.29) } \quad T U=2 \pi i\left(n_{1} e_{1}+\ldots+n_{s} e_{g}\right)+m_{1} f_{1}+\ldots+m_{g} f_{g},
$$

where $2 \pi i e_{1}, \ldots, 2 \pi e_{g}, f_{1}, \ldots, f_{g}$ are basis vectors of the period lattice, the $n_{k}$ and $m_{j}$ are integers, and $T \neq 0$, then $u(x, y, t)$ is periodic in $x$ with 
period $T$. If in addition we can find a second (complex) period $T^{\prime}$, where $\operatorname{Im}\left(T^{\prime} / T\right) \neq 0$ such that

$$
\text { (3.1.30) } \quad T^{\prime}(U)=2 \pi i\left(n_{\mathbf{1}}^{\prime} e_{1}+\ldots+n_{\mathbf{g}}^{\prime} e_{\mathbf{g}}\right)+m_{\mathbf{1}}^{\prime} f_{1}+\ldots+m_{\boldsymbol{g}}^{\prime} f_{g},
$$

where the $n_{k}^{\prime}$ and $m_{j}^{\prime}$ are integers, then $u(x, y, t)$, as a function of $x$, is a doubly periodic meromorphic function (with the periods $T$ and $T^{\prime}$ ) and can be expressed in terms of elliptic functions.

Example. (see [53]). For the Riemann surface $\Gamma$ of the second kind, given by the equation

$$
\boldsymbol{w}^{2}=z^{3}-\frac{21}{4} g_{2} z^{3}-\frac{27}{4} g_{3} z^{2}+\frac{27}{4} g_{2}^{2} z+\frac{81}{4} g_{2} g_{3},
$$

and for the Weierstrass point $Q=\infty$ on $\Gamma$, the dependence on $y$ disappears, and the $\mathrm{KP}$ equation reduces to the $\mathrm{KdV}$ equation (see below) whose solution can be expressed in terms of elliptic functions by the formulae

$$
u(x, t)=2 \wp\left(x-x_{1}(t)\right)+2 \wp\left(x-x_{2}(t)\right)+2 \wp\left(x-x_{3}(t)\right) .
$$

Here $\varnothing(x)$ is the Weierstrass function (see Ch. I, §3),

$$
\begin{gathered}
\left(8^{\prime}\right)^{2}=48^{83}-g_{2} 8-g_{3}, \quad x_{1}+x_{2}+x_{3}=\text { const }, \quad-4 t=\int_{0}^{x_{1}-x_{2}} \frac{d z}{\sqrt{12\left(g_{2}-3 y^{\prime 2}(z)\right)}}, \\
x_{2}-x_{3}=\frac{1}{2} \wp^{-1}\left[-8\left(x_{1}-x_{3}\right)+\sqrt{g_{2}-38^{2}\left(x_{1}-x_{3}\right)}\right] .
\end{gathered}
$$

In particular, if $t=0$, const $=0$, then $u(x, 0)=6 \wp^{\circ}(x)$. An elegant method of finding the Riemann surfaces for which the function $u=2 \partial^{2} / \partial x^{2} \log \theta$ can be expressed by elliptic functions has recently been found by Krichever [22] .

Suppose that the Riemann surface $\Gamma$ and the point $Q$ on it are such that there exists a meromorphic function $\lambda(P)$ on $\Gamma$ with a unique double pole at $Q$. It is easy to see that then $\Gamma$ must be hyperelliptic and $Q$ must be a Weierstrass point (a branch point). We choose $k^{-1}(P)=[\lambda(P)]^{-1 / 2}$ as the local parameter of $k^{-1}=k^{-1}(P)$ in a neighbourhood of $P=Q$. Then the Baker-Akhiezer function $\psi(x, y, t ; P)$ with as essential singularity $\exp \left(k x+k^{2} y+k^{3} t\right)$ at $Q$ has the form

$$
\psi(x, y, t ; P)=\exp (y \lambda(P) \varphi(x, t ; P)),
$$

where $\psi$ is the Baker-Akhiezer function with the same divisor of poles as $\psi$, and with an essential singularity $\varphi \sim \exp \left(k x+k^{3} t\right)$ at $Q$. This follows immediately from the uniqueness Theorem 3.1.1. Then the differential equations (3.1.15), (3.1.16) for $\varphi$ can be rewritten as follows:

$$
\begin{gathered}
L \varphi(x, t ; P)=\lambda(P) థ(x, t ; P), \\
\frac{\partial \varphi}{\partial t}=A \varphi .
\end{gathered}
$$


Now (3.1.34) means that $\varphi$ is the "eigenfunction" of the Schrödinger (Sturm-Liouville) operator ${ }^{(1)} L$ with eigenvalue $\lambda(P)$, depending on the parameter $t$, by (3.1.35). The coefficients of the operators $L$ and $A$ are independent of $y$, and the KP equation turns into the Korteweg-de Vries $(\mathrm{KdV})$ equation

$$
u_{t}=\frac{1}{4}\left(6 u u_{x}+u_{x x x}\right) .
$$

The commutation condition (3.1.19) gives the " $L-A$ pair" for the KdV equation

$$
\frac{d L}{d t}=[A, L]
$$

and finally, (3.1.25) gives the standard formula (of Matveev-Its) for finitezone solutions of the equation

$$
u(x, t)=2 \frac{\partial^{2}}{\partial x^{2}} \log \theta\left(x U+t W+z_{0}\right)+c,
$$

where $U$ and $W$ were defined above.

Similarly, if for a curve $\Gamma$ and a point $Q$ on it there exists a meromorphic function $\mu(P)$ with a single pole of the third order at $Q$, then the dependence on $t$ disappears and the KP equation turns into a version of the equation of a non-linear string (the Boussinesq equation)

$$
3 u_{y y}+\frac{\partial}{\partial x}\left(6 u u_{x}+u_{x x x}\right)=0 .
$$

which has solutions of the form

$$
u(x, y)=2 \frac{\partial^{2}}{\partial x^{2}} \log 0\left(x U+y V+z_{0}\right)+c .
$$

We now indicate a set of conditions that are sufficient for the constructed solutions of the following equation to be real

$$
-\frac{3}{4} u_{y y}=\frac{\partial}{\partial x}\left[u_{t}-\frac{1}{4}\left(6 u u_{x}+u_{x x x}\right)\right],
$$

which is obtained from the KP equation by the substitution $y \mapsto i y$. Let $\tau$ be an anti-involution (that is, an anti-holomorphic automorphism $\tau: \Gamma \rightarrow$ $\rightarrow \Gamma, \tau^{2}=1$ ) on $\Gamma$ of genus $g=2 \rho+n-1$, having $n$ fixed cycles $A_{0}, \ldots A_{n-1}$. When we cut $\Gamma$ along the cycles $A_{0}, \ldots, A_{n-1}$, we obtain two connected components $\Gamma^{+}$and $\Gamma^{-}=\tau\left(\Gamma^{+}\right)$, each of which is an open Riemann surface of genus $\rho$ with boundary consisting of the cycles $A_{0}, \ldots, A_{n-1}$. Then in the homology group $H_{1}(\Gamma ; \mathbf{Z})$ we can then choose the canonical basis of cycles

$$
a_{1}, b_{1}, \ldots, a_{\rho}, b_{\rho}, a_{\rho+1}, b_{\rho+1}, \ldots, a_{\rho+n-1}, b_{\rho+n-1}, a_{1}^{\prime}, b_{1}^{\prime}, \ldots, a_{\rho}^{\prime}, b_{\rho}^{\prime}
$$

\footnotetext{
(1) Under suitable conditions on the Riemann surface $\Gamma$ and the divisor $D$ on $\Gamma$, the potential $u$ of the Schrödinger operator is a real almost-periodic function. In this case $L$ is an operator with regular analytic properties, that is, has an eigenfunction $\varphi$ that is meromorphic on $\Gamma$ (see [17]).
} 
such that $a_{\mathrm{p}+k}=A_{k}(k=1, \ldots, n-1)$, and

$$
\begin{gathered}
a_{i}, b_{i} \in \Gamma^{+}, a_{i}^{\prime}, \quad b_{i}^{\prime} \in \Gamma^{-} ; \quad \tau\left(a_{i}\right)=a_{i}^{\prime}, \quad \tau\left(b_{i}\right)=-b_{i}^{\prime} \quad(i=1, \ldots, \rho), \\
\tau\left(a_{\rho+k}\right)=a_{\rho+k}, \quad \tau\left(b_{\rho+k}\right)=-b_{\rho+k} \quad(k=1, \ldots, n-1) .
\end{gathered}
$$

To obtain real solutions, the essential singularity $Q$ of the Baker-Akhiezer function must be chosen fixed with respect to $\tau$ (that is, $\tau(Q)=Q$ ), and the local parameter $z$ in a neighbourhood of $Q$ (where $z(Q)=0$ ) so that $\tau(z)=-\bar{z}$. Such a point $Q$ and local parameter $z$ determine the vectors $U, V$, and $W$ (see above). Then the smooth real solutions of (3.1.41) have the form

$$
\text { (3.1.42) } u(x, y, t)=2 \partial_{x}^{2} \log \theta\left(x U+i y V+t W+z_{0}\right)+c,
$$

where $z_{0}$ has the form

$$
z_{0}=\left(z_{0}^{\prime}, z_{0}^{\prime \prime}, \bar{z}_{0}^{\prime}\right), \quad z_{0}^{\prime} \in \mathbf{C}^{\rho}, z_{0}^{\prime \prime} \in \mathbf{R}^{n-1},
$$

and $c$ is a real constant (this can be deduced from [8], for example).

Remark. The Baker-Ahkiezer function $\psi(x, y, t ; P)$ having poles on $\Gamma \backslash Q$, also has $g$ zeros there (for almost all $x, y, t$ ). These zeros depend on the parameters $x, y$, and $t$. We denote them by $Q_{1}(x, y, t), \ldots, Q_{g}(x, y, t)$. The dependence of these zeros on $x, y$, and $t$ can be determined from the following proposition (Akhiezer).

Lemma 3.1.2. For the zeros $Q_{1}=Q_{1}(x, y, t), \ldots, Q_{g}=Q_{g}(x, y, t)$ and poles $P_{1}, \ldots, P_{g}$ of the Baker-Akhiezer function $\psi(x, y, t)$ the following relation holds on the Jacobi variety $J(\Gamma)$ :

$$
\begin{aligned}
A\left(Q_{1}\right)+\ldots+A\left(Q_{g}\right) \equiv A\left(P_{1}\right)+\ldots+A\left(P_{g}\right) & +U x+ \\
& +V y+W t
\end{aligned}
$$

where the vectors $U, V$, and $W$ are determined above.

The proof of the lemma repeats almost word for word that of Abel's theorem. The periods of the meromorphic differential $d \log \psi$ must be integral multiples of $2 \pi i$. This differential can be represented as a linear combination of elementary ones (see Ch. II, $\S 1$ ):

$$
d \cdot \log \psi=\sum_{i=1}^{g} \omega_{Q_{i} P_{i}}+x \Omega_{Q}^{(1)}+y \Omega_{Q}^{(2)}+t \Omega_{Q}^{(3)}+\sum_{i=1}^{g} c_{i} \omega_{i} .
$$

Integrating this expression over all the cycles $a_{j}$ and $b_{k}$, we obtain (3.1.43).

From this lemma one can derive another proof of Theorem 3.1.1.

Differentiating (3.1.43) with respect to $x, y, t$ we obtain differential equations describing the dynamics of the zeros $\left(Q_{1}, \ldots, Q_{g}\right)$. For example, for the hyperelliptic surface $\Gamma$ of genus $2\left\{w^{2}=P_{5}(z)\right\}$ and for the Weierstrass point $Q=\infty$ on $\Gamma$ the dependence on $x$ of the zeros $\left(Q_{1}, Q_{2}\right)$ is defined by (2.4.55) (up to a factor of 2). The dependence on $y$ disappears and the dependence on $t$ is given by a linear combination of (2.4.55) and (2.4.56). 
We complete this section by the following general observation. The polynomial $q(k)=k x+k^{2} y+k^{3} t$ from which the Baker-Akhiezer function was constructed was the simplest possible. It is easy to generalize the calculations and to prove the following proposition.

Theorem 3.1.4. Let $\Gamma$ be a Riemann surface of genus $g, Q$ a point on $\Gamma$, and $k^{-1}$ a local parameter in a neighbourhood of $Q$. We fix a non-special divisor $D$ of degree $g$.

a) Each polynomial $q(k)$ of degree $n$ determines an ordinary differential operator $L_{q}$ (in $x$ ) of the $n$-th order, according to the following rule.

Suppose that $\psi_{q}=\psi_{q}(x, y ; P)$ is the Baker-Akhiezer function with divisor $D$ of the poles and with the essential singularity $\exp (k x+q(k) y)$ at $Q$. Then $L_{q}$ is uniquely determined by

$$
\frac{\partial \psi_{q}}{\partial y}=L_{q} \psi_{q} \cdot
$$

The coefficients of $L_{q}$ can be expressed recurrently in terms of the coefficients of the series $\psi_{q} \exp (-k x-q(k) y)$ in reciprocal powers of $k$.

b) Every pair of polynomials $q(k)$ and $r(k)$ generates a solution of a nonlinear equation in coefficients of $L_{q}$ and $L_{r}$ of the form

$$
\text { (3.1.46) } \quad\left[-\frac{\partial}{\partial y}+L_{q},-\frac{\partial}{\partial t}+L_{r}\right]=0 \text {. }
$$

This solution can be expressed in terms of the $\theta$-function of $\Gamma$.

c) In particular, every meromorphic function $\lambda(P)$ with a single pole at $P=Q$ gives an operator of the form $L=L_{\lambda}$ and a set of eigenfunctions $\psi$,

$$
L \psi=\lambda \psi,
$$

if we take $q(k)$ to be principal part of the Laurent series of this function at $Q$ and $L=L_{q}$. In this case any other polynomial $r(k)$ determines a solution of a non-linear equation in the coefficients of $L$ of the form

$$
\frac{\partial L}{\partial t}=[A, L], \quad A=L_{r},
$$

which can also be expressed by $\theta$-functions.

d) A pair of meromorphic functions $\lambda(P), \mu(P)$ with a single pole at $P=Q$ gives rise to a pair of commuting ordinary differential operators

$$
[A, L]=0 .
$$

The equations with the coefficients of these operators are called the Novikov equations and can also be integrated by means of $\theta$-functions.

One can also reformulate c) and d) of this theorem as follows: every nonspecial divisor $D$ of degree $g$ gives rise to a homomorphism of the ring of the meromorphic functions with a single pole at $Q$ into a commutative algebra of ordinary differential operators

$$
\lambda(P) \mapsto L_{\lambda}
$$


All the operators of the form $L_{\lambda}$ have a common eigenfunction $\psi$,

$$
L_{\lambda} \psi=\lambda \psi,
$$

which is the Baker-Akhiezer function with divisor $D$ and essential singularity $\exp k x$ at $Q$.

For a proof of this theorem as well as other applications of it and a generalization to matrix and difference operators, see [15] and [16].

\section{$\S 2$. The Baker-Akhiezer two-point function. The Schrödinger equation in a magnetic field}

The simplest generalization of the Baker-Akhiezer function we have studied in the preceding section consists in adding superfluous essential singularities. The general definition of the Baker-Akhiezer 1-point functions ("of rank 1") is as follows.

Definition 3.2.1. Let $\Gamma$ be a Riemann surface of genus $g, Q_{1}, \ldots, Q_{l}$ points on $\Gamma ; k_{1}^{-1}, \ldots, h_{i}^{-1}$ local parameters in a neighbourhood of these points (where $k_{i}\left(Q_{i}\right)=\infty$ ), $q_{1}(k), \ldots, q_{1}(k)$ a set of polynomials, $D$ a divison on $\Gamma \backslash\left(Q_{1} \cup \ldots \cup\left(Q_{l}\right)\right.$, and $\psi=\psi(P)$ a Baker-Akhiezer $l$-point function specified by these data: it is meromorphic on $\Gamma \backslash\left(Q_{1} \cup \ldots \cup Q_{l}\right)$ and such that a) the divisor of $\psi \geqslant-D ;$ b) as $P \rightarrow Q_{i}$, the product $\psi(P) \exp \left(-q_{i}\left(k_{i}(P)\right)\right)$ is analytic $(i=1, \ldots, l)$.

The Baker-Akhiezer functions given by the conditions of this definition form a linear space, which we denote by $\Lambda_{l}(D)$. By analogy with Theorem 3.1.1 one can prove the following theorem.

Theorem 3.2.1. Let $\Gamma$ be a Riemann surface of genus $g$ and $D$ a non-special divisor on $\Gamma \backslash\left(Q_{1} \cup \ldots \cup Q_{l}\right)$. Then the dimension of the space $\Lambda_{l}(D)$ is $\operatorname{deg} D-g+1$. In particular, if $D=P_{1}+\ldots+P_{g}$ is a non-special divisor of degree $g$, then the corresponding Baker-Akhiezer l-point function exists and is uniquely determined up to a factor.

The use of the Baker-Akhiezer multipoint functions allows us to integrate a very large number of importart non-linear equations. We analyse a simple example of the use of the Baker-Akhiezer two-point funtion, without giving here a general theory of applying multipoint functions to the integration of equations (see [15] and [16]).

Let $\Gamma$ be an arbitrary Riemann surface of genus $g, Q_{+}, Q_{-}$a pair of points on $\Gamma$, and $k^{+}$and $k^{-}$local parameters in neighbourhoods of $Q_{+}$and $Q_{-}$. We consider the Baker-Akhiezer function $\psi(z, \bar{z} ; P)$ with the divisor $D$ of poles, where $D=P_{1}+\ldots+P_{g}$ is a non-special divisor of degree $g$ on $\Gamma \backslash\left(Q_{+} \cup Q_{-}\right)$ and with essential singularities of the form $\exp k_{+} z$ and $\exp k_{-} z$ at $Q_{+}$and $Q_{-}$, respectively. Here $z=x+i y$ and $\bar{z}=x-i y$ are independent variables. 
Such a function exists and is uniquely determined up to normalization. Its explicit expression in terms of the $\theta$-function of $\Gamma$ is as follows:

$$
\psi(z, \bar{z} ; P)=\exp \left(z \int_{P_{0}}^{P} \Omega_{+}+\bar{z} \int_{P_{0}}^{P} \Omega_{-}\right) \frac{\theta\left(A(P)-A(D)+z U_{+}+\bar{z} U_{-}-K\right)}{\theta(A(P)-A(D)-K)} .
$$

Here $\Omega_{+}$and $\Omega_{-}$are normalized differentials of the second kind with a double pole at $Q_{+}$and $Q_{-}$, respectively, and principal parts at these points of the form

$$
\Omega_{ \pm}=d\left(k_{ \pm}\right)+\ldots ;
$$

$U_{+}$and $U_{-}$are the vectors of the $b$-periods of these differentials,

$$
U_{ \pm_{i}}=\oint_{b_{i}} \Omega_{ \pm} \quad(i=1, \ldots, g) .
$$

In neighbourhoods of $Q_{ \pm}$the function $\psi(z, \bar{z} ; P)$ has an expansion of the form: as $P \rightarrow Q_{+}$,

$$
\psi=c_{+} e^{k z}\left(1+\frac{\text { s.t }_{1}^{+}}{k}+\frac{\xi_{2}^{+}}{k^{2}}+\ldots\right), \quad k=k_{+},
$$

and as $P \rightarrow Q_{-}$,

$$
\Psi=c_{-} e^{k \bar{z}}\left(1+\frac{\bar{\xi} \overline{1}}{k}+\frac{\bar{s} \bar{g}}{k^{2}}+\ldots\right), \quad k=k_{-} .
$$

The coefficients $c_{ \pm}$and $\xi_{i}^{ \pm}$are functions of $z$ and $\bar{z}$. We normalize $\psi$ so that the coefficient $c_{ \pm}$in (3.2.4) is 1 . Then we denote the coefficient $c_{-}$by $c\left(c=c_{-} / c_{+}\right)$. By analogy with Theorem 3.1.2 we can prove the next theorem.

Theorem 3.2.2. The Baker-Akhiezer function $\psi(z, \bar{z} ; P)$, normalized by the condition $c_{+}=1$, is a solution of the equation

$$
H \psi=0,
$$

where

$$
H=-4 \frac{\partial^{2}}{\partial z \overrightarrow{\partial z}}+a(z, \bar{z}) \frac{\partial}{\partial \bar{z}}+b(z, \bar{z}),
$$

and the coefficients $a, b$ of the operator $H$ have the form $\left(c=c_{-} / c_{+}\right)$

$$
a=4 \frac{\partial \log c}{\partial z}, \quad b=4 \frac{\partial \xi_{1}^{+}}{\partial \bar{z}} .
$$

Proof. The form of $H$, by analogy with Lemma 3.1.1, is selected from the following two conditions: for the expansion (3.2.4) (with $c_{+}=1$ ):

$$
H y=O\left(\frac{1}{k}\right) e^{k z}
$$

for the expansion (3.2.5):

$$
H_{\psi}:=e^{k \bar{z}}\left(\tilde{c}(z, \bar{z})+O\left(\frac{1}{k}\right)\right)
$$


(the explicit form of $\widetilde{c}(z, \bar{z})$ is not important for us). From (3.2.9) and (3.2.10) it follows that $H \psi$ is again a Baker-Akhiezer function, but at $P=Q_{+}$the product $(H \psi) \exp \left(-k_{+} z\right)$ vanishes. By the uniqueness, $H \psi=0$. This proves the theorem.

The operator $H$ constructed from $\Gamma$, the pair of points $Q_{ \pm}$on $\Gamma$, and the divisor $D$ can be interpreted as the Schrödinger operator for a twodimensional electron in the magnetic field $B(x, y)=\partial_{x} A_{y}-\partial_{y} A_{x}$, where $\left(A_{x}, A_{y}\right)$ is a vector-potential, in the presence of a potential $u(x, y)$ (of the electric field):

$$
\begin{gathered}
H=\left(i \frac{\partial}{\partial x}+e A_{x}\right)^{2}+\left(i \frac{\partial}{\partial y}+e A_{y}\right)^{2}+u(x, y), \\
\frac{\partial}{\partial x}=\frac{\partial}{\partial z}+\frac{\partial}{\partial \bar{z}}, \quad \frac{\partial}{\partial y}=i\left(\frac{\partial}{\partial z}-\frac{\partial}{\partial \bar{z}}\right) ;
\end{gathered}
$$

$e$ is the electron charge; $\hbar=2 m=c=1$ (the magnetic field $B(x, y)$ is directed along the third axis). For the operator (3.2.7) we have

$$
\begin{aligned}
& \left\{\begin{array}{l}
2 i e\left(A_{x}-i A_{y}\right)=a(z, \bar{z})=4 \frac{\partial \log c}{\partial z}, \\
2 i e\left(A_{x}+i A_{y}\right)=0
\end{array}\right. \\
& u=b+\frac{1}{2} \frac{\partial a}{\partial \bar{z}}=4 \frac{\partial \xi_{i}^{+}}{\partial \bar{z}}+2 \frac{\partial^{2} \log c}{\partial z \partial \bar{z}} .
\end{aligned}
$$

We now deduce formulae expressing the coefficients $a, b$ (and thus, the magnetic field $B=\partial_{x} A_{y}-\partial_{y} A_{x}$ and the electric field $\left.u(x, y)\right)$ in terms of $\theta$-functions.

Theorem 3.2.3. The function

$$
\begin{aligned}
\psi(z, \bar{z} ; P)=\exp \left[z\left(\int_{P_{0}}^{P} \Omega_{+}-\alpha_{+}\right)+\bar{z}\left(\int_{P_{0}}^{P} \Omega_{-}-\alpha_{-}\right)\right] \times \\
\quad \times \frac{\theta\left(A(P)+z U_{+}+\bar{z} U_{-}+\zeta_{0}\right) \theta\left(A\left(P_{+}\right)+\zeta_{0}\right)}{\theta\left(A(P)+\zeta_{0}\right) \theta\left(A\left(P_{+}\right)+z U_{+}+\bar{z} U_{-}+\zeta_{0}\right)},
\end{aligned}
$$

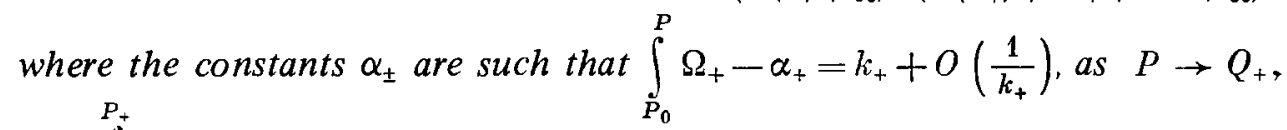
$\alpha_{-}=\int_{\dot{P}_{0}}^{P_{+}} \Omega_{-}$, for any $\xi_{0}$ is a solution of the equation $H \psi=0$, where $H$ is the

Schrödinger operator of a two-dimensional electron in a magnetic field,

$$
H=\left(i \frac{\partial}{\partial x}+e A_{x}\right)^{2}+\left(i \frac{\partial}{\partial y}+e A_{y}\right)^{2}+u(x, y) .
$$

The coefficients of this operator have the form

(3.2.16) $\quad u(x, y)=$

$=2 \frac{\partial^{2}}{\partial z \partial \bar{z}} \log \left[\theta\left(A\left(P_{+}\right)+z U_{+}+\bar{z} U_{-}+\zeta_{0}\right) \theta\left(A\left(P_{-}\right)+z U_{+}+\bar{z} U_{-}+\zeta_{0}\right)\right]+$ const $_{r}$

$$
A_{x}=-i A_{y}=\frac{1}{i e} \frac{\partial}{\partial z} \log \frac{\theta\left(A\left(P_{-}\right)+z U_{+}+\bar{z} U_{-}+\zeta_{0}\right)}{\theta\left(A\left(P_{+}\right)+z U_{+}+\bar{z} U_{-}+\zeta_{0}\right)} .
$$


The magnetic field $B(x, y)=\partial_{x} A_{y}-\partial_{y} A_{x}$ is directed along the third axis and has the form

(3.2.18) $\quad B(x, y)=\frac{2}{e} \frac{\partial^{2}}{\partial z \partial \bar{z}} \log \frac{\theta\left(A\left(P_{-}\right)+z U_{+}+\bar{z} U_{-}+\zeta_{0}\right)}{\theta\left(A\left(P_{+}\right)+z U_{+}+\bar{z} U_{-}+\zeta_{0}\right)}$.

Proof. (3.2.14) is obtained from (3.2.1) by normalization (we divide by $c_{+}$), where $\xi_{0}=-A(D)-K$ is an arbitrary point of the Jacobi variety $J(\Gamma)$. Then for the functions $c(z, \bar{z})$ and $\xi_{1}^{+}$we have

$$
\begin{aligned}
& \log c(z, \bar{z})=\log \frac{\theta\left(A\left(P_{-}\right)+z U_{+}+\bar{z} U_{-}+\zeta_{0}\right) \theta\left(A\left(P_{+}\right)+\zeta_{0}\right)}{\theta\left(A\left(P_{-}\right)+\zeta_{0}\right) \theta\left(A\left(P_{+}\right)+z U_{+}+\bar{z} U_{-}+\zeta_{0}\right)}+\ldots \\
& \xi_{1}^{+}=-\frac{\partial}{\partial z} \log \frac{\theta\left(A\left(P_{+}\right)+z U_{+}+\bar{z} U_{-}+\zeta_{0}\right)}{\theta\left(A\left(P_{+}\right)+\zeta_{0}\right)}+\ldots
\end{aligned}
$$

where the dots denote linear combinations of the variables $z$ and $\bar{z}$, arising from the expansion of the exponent of the exponential curve in (3.2.14). This and (3.2.12) and (3.2.13) immediately prove the theorem.

Remark. The group of periods of the magnetic field $B(x, y)$ is the same as that of the vector-potential $\left(A_{x}, A_{y}\right)$-this follows from the explicit formulae obtained. Therefore, in a doubly periodic field (a two-dimensional crystal lattice with periods $T_{x}$ and $T_{y}$ ) the constructed solutions have a zero flux through the nucleus of the lattice $\int_{0}^{T_{v}} \int_{0}^{T_{y}} B(x, y) d x d y=0$. The case of nonzero flux has been integrated in [37].

There is a simple sufficient condition for the constructed operators $H$ to be real (see [49]).

Lemma 3.2.1. Suppose that there is an anti-involution $\tau$ on $\Gamma$, permuting $Q_{+}$and $Q_{-}$

$$
\tau: \Gamma \rightarrow \Gamma, \tau^{2}=1, \tau\left(Q_{+}\right)=Q_{-} .
$$

We choose local parameters $k_{+}, k_{-}$in neighbourhoods of these points so that $k_{-}=-\overline{\tau\left(k_{+}\right)}$. If the divisor $D$ (non-special of degree $g$ ) is such that $D+\tau(D)$ is a zero divisor of a differential $\omega$ of the third kind with simple poles at $Q_{+}$and $Q_{-}$, then the coefficients of $H$ are real.

Proof. By the condition of the lemma, the product $\tilde{\omega}:=\psi(z, \bar{z} ; P) \times$ $\times \overline{\psi(z, \bar{z} ; \tau(P))} \omega$ is again a differential of the third kind with simple poles at $Q_{+}$and $Q_{-}$. Since the sum of its residues in zero, we have $c=\bar{c}$, from which it follows that $B$ is real. That $u$ is real is verified analogously.

By (2.4.20), the condition on $D$ in this lemma can be rewritten in the form of a relation on the Jacobian variety $J(\Gamma)$ :

$$
A(D)+A(\tau(D))=A\left(Q_{+}\right)+A\left(Q_{-}\right)-2 K
$$

( $K$ are the Riemann constants). 


\section{EFFECTIVIZATION OF THE FORMULAE FOR THE SOLUTION OF KdV AND KP EQUATIONS. RECOVERY OF A RIEMANN SURFACE FROM ITS JACOBI VARIETY. THE PROBLEM OF RIEMANN AND THE CONJECTURE OF NOVIKOV}

\section{$\S 1$. The $\mathrm{KdV}$ equation. Genus $g=1$ or 2}

In the preceding chapter we have obtained "explicit formulae" (3.1.25) and (3.1.38) expressing the solutions of a number of important non-linear equations in terms of $\theta$-functions. These formulae are of little use for calculations within the framework of the theory of $\theta$-functions for the following two reasons:

1) The Riemann matrix $B_{j k}$ is not arbitrary;

2) The connection between the vectors $U, V, W$ and the Riemann matrix $B_{j k}$ is transcendental (this connection has never been discussed explicitly).

We recall (see $\mathrm{Ch}$. II, $\S 1$ ) that the set of period matrices ( $B_{j k}$ ) of Riemann surfaces of genus $g>1$ depends on $3 g-3$ complex parameters (for $g=1$ on one parameter). However, the general $(g \times g)$-Riemann matrices form a family of dimension $g(g+1) / 2$. Riemann raised the question: what conditions have to be imposed on a Riemann matrix $B_{j k}$ so that it is a period matrix of holomorphic differentials on a Riemann surface $\Gamma$ ? For the genera $g=1,2,3$ the Riemann matrix can be any indecomposable matrix. Riemann's problem is non-trivial for $g \geqslant 4$ and an effective solution of it for all $g \geqslant 4$ has not yet been obtained (see [26], [38], [39]).

Novikov suggested obtaining a full set of necessary relations on the matrix $B_{j k}$ and the vectors $U, V, W$ via a simple substitution of (3.1.38) and (3.1.25) in the KdV and the KP equations, where the $\theta$-function is determined by its Fourier series (1.1.1). Then one obtains a system of algebraic equations from which the vectors $U, V, W$ can be determined; the compatibility conditions of this system give a full set of relations on the Riemann matrix $B_{j k}$.

The present chapter is devoted to a realization of this problem. In particular, for $g=1,2,3$ (where the Riemann matrix $B_{j k}$ is arbitrary) a complete effectivization of $(3.1 .25),(3.1 .28)$ is given to solve the KP equation and the equations connected with it.

We start with the case of small genera $g=1$ or 2 for the $\mathrm{KdV}$ equation. Here the Riemann matrix $B_{j k}$ is arbitrary (in general position). We look for a solution of the $\mathrm{KdV}$ equation

$$
u_{t}=\frac{1}{4}\left(6 u u_{x}+u_{x x x}\right)
$$

in the form

$$
u(x, t)=2 \frac{\partial^{2}}{\partial x^{2}} \log \theta\left(U x+W t+z_{0}\right)
$$


where the $\theta$-function is constructed from some Riemann $(g \times g)$-matrix $B_{j k}$, the vectors are unknown, and $z_{0}$ is an arbitrary $g$-dimensional vector. This formula differs from (3.1.38) by the fact that $c=0$, which can always be achieved by the change $W \mapsto W-\frac{2 c}{3} U$. Substituting (4.1.2) in (4.1.1) we obtain

$$
2 \frac{\partial^{3}}{\partial x^{2} \partial t} \log \theta=\frac{1}{4}\left[24 \frac{\partial^{2} \log \theta}{\partial x^{2}} \frac{\partial^{3} \log \theta}{\partial x^{3}}+2 \frac{\partial^{5} \log \theta}{\partial x^{5}}\right],
$$

where $\theta=\theta(z), z=U x+W t+z_{0}$ is an arbitrary vector, $\frac{\partial}{\partial x}=\sum U_{i} \frac{\partial}{\partial z_{i}}$, and $\frac{\partial}{\partial t}=\sum W_{i} \frac{\partial}{\partial z_{i}}$. This expression can be written in the form

$$
\frac{\partial}{\partial x}\left\{-2 \frac{\partial^{2} \log \theta}{\partial x \partial t}+3\left(\frac{\partial^{2} \log \theta}{\partial x^{2}}\right)^{2}+\frac{1}{2} \frac{\partial^{2} \log \theta}{\partial x^{4}}\right\}=0 .
$$

Suppose that $B_{j k}$ is indecomposable (see Ch. I, §3). In this case it follows from (4.1.3) that the expression in curly brackets is a constant, which we denote by $4 d$. An elementary calculation leads us to the relation

$$
\text { (4.1.4) } \quad \theta_{x x x x} \theta-4 \theta_{x x x} \theta_{x}+3 \theta_{x x}^{2}-4 \theta_{x t} \theta+4 \theta_{x} \theta_{t}+8 d \theta^{2}=0
$$

(the subscripts $x$ and $t$ denote derivatives), where $\theta=\theta(z)$, which must hold for any $z$. To obtain a finite system of equations in $U, W$, and the constant $d$, we use the addition Theorem 1.4.1. In our special case it has the form

$$
\begin{gathered}
\theta\left(z^{1}\right) \theta\left(z^{2}\right)=\sum_{n \in \frac{1}{2}\left(\mathbf{z}_{2}\right)^{g}} \hat{\theta}[n]\left(w^{1}\right) \hat{\theta}[n]\left(w^{2}\right), \\
z^{1}+z^{2}=w^{1}, \quad z^{1}-z^{2}=w^{2} .
\end{gathered}
$$

Here we use the abbrevation

$$
\hat{\theta}[n](w)=\theta[n, 0](w \mid 2 B) .
$$

The notation $n \in \frac{1}{2}\left(\mathbf{Z}_{2}\right)^{g}$ means that the summation in (4.1.5) is over all of the half-periods $n=\left(n_{1}, \ldots, n_{g}\right), n_{i}=0, \frac{1}{2}$.

The values of the functions $\hat{\theta}[n](w)$ and their derivatives

$$
\hat{\theta}_{i j} \ldots[n](w) \equiv \frac{\partial}{\partial w_{i}} \frac{\partial}{\partial w_{j}} \ldots \hat{\theta}[n](w)
$$

at $w=0$ are called $\theta$-constants. We agree to omit a zero argument of the $\theta$-constants: $\hat{\theta}_{i j} \ldots[n] \equiv \hat{\theta}_{i j}[n](0)$.

Lemma 4.1.1. (4.1.4) is equivalent to the following system of $2^{g}$ equations in the vectors $U=\left(U_{1}, \ldots, U_{g}\right), W=\left(W_{1}, \ldots, W_{g}\right)$, and the constant $g$ :

$$
\partial_{U}^{4} \hat{\theta}[n]-\partial_{v} \partial_{w} \hat{\theta}[n]+d \hat{\theta}[n]=0,
$$

where the equations are numbered by $n \in \frac{1}{2}\left(\mathbf{Z}_{2}\right)^{g}$. Here we introduce the notation

$$
\left\{\begin{array}{l}
\partial_{U}^{4} \theta[n]=\sum_{i, j, k, l} U_{i} U_{j} U_{k} U_{l} \hat{\theta}_{i j k l}[n], \\
\partial_{U} \partial_{W} \hat{\theta}[n]=\sum_{i, j} U_{i} W_{j} \hat{\theta}_{i j}[n] .
\end{array}\right.
$$


Proof. We introduce operators

$$
\begin{cases}X_{z^{1}}=\sum U_{j} \frac{\partial}{\partial z_{j}^{1}}, & X_{z^{2}}=\sum U_{j} \frac{\partial}{\partial z_{j}^{2}} \\ T_{z^{1}}=\sum W_{j} \frac{\partial}{\partial z_{j}^{1}}, & T_{z^{2}}=\sum W_{j} \frac{\partial}{\partial z_{j}^{2}}\end{cases}
$$

and, by analogy, operators $X_{w^{1}}, X_{w^{2}}, T_{w^{1}}, T_{w^{2}}$, where all $z$ are substituted for $w$, connected by the relations (see $\left(4.1 .5^{\prime}\right)$ )

$$
\begin{cases}X_{z^{1}}=X_{w^{1}}+X_{w^{2}}, & T_{z^{1}}=T_{w^{1}}+T_{w^{2}}, \\ X_{z^{2}}=X_{w^{1}}-X_{w^{2}}, & T_{z^{2}}=T_{w^{1}}-T_{w^{2}} .\end{cases}
$$

Now (4.1.4) can be rewritten in the form

$$
\begin{aligned}
{\left[\left(X_{z}^{4} 1-4 X_{z}^{3} X_{z}+3 X_{z^{1}}^{2} X_{z^{2}}^{2}+\right.\right.} & 4 X_{z_{1}} T_{z^{2}}- \\
& \left.\left.-4 X_{z^{1}} T_{z^{1}}+8 d\right) \theta\left(z^{1}\right) \theta\left(z^{2}\right)\right]_{z^{1}=z^{2}}=0 .
\end{aligned}
$$

We express the operator in square brackets in terms of the $X_{w^{i}}$ and $T_{u^{i}}$ in (4.1.9) and apply it to the right-hand side of the addition Theorem (4.1.5) for $w^{1}=2 z, w^{2}=0$. Since $\hat{\theta}[n](w)$ is even, it suffices to leave the even powers of $X_{w^{2}}$ and $T_{w^{2}}$ in the resulting expression. If we take this into consideration, the calculation becomes very simple and leads to the relation

$$
\left[8\left(X_{w^{2}}^{4}-X_{w^{2}} T_{w^{2}}+d\right) \sum_{n \in \frac{1}{2}\left(\mathbf{z}_{2}\right)^{g}} \hat{\theta}[n]\left(u^{1}\right) \hat{\theta}[n]\left(w^{2}\right)\right]_{\substack{w^{1}=2 z \\ w^{2=0}}}^{2=0}=0 .
$$

It is easy to see that the $2^{g}$ functions $\theta[n](2 z), n \in \frac{1}{2}\left(Z_{2}\right)^{g}$, are linearly independent (they form a basis in the space of the $\theta$-functions of the second order-see Ch. I, §1). Equating the coefficients of these functions equal to zero we obtain the system (4.1.6). This proves the lemma.

The system (4.1.6) is invariant under the following scale transformations:

$$
U \mapsto \lambda U, W \mapsto \lambda^{2} U, \quad d \mapsto \lambda^{4} d .
$$

It is easy to compare these transformations with the obvious scale group of the $\mathrm{KdV}$ equation.

Let us now solve the system (4.1.6) for $g=1$ and 2 .

a) ${ }^{(1)} g=1$. Then (4.1.6) takes the form:

$$
\left\{\begin{array}{l}
U^{4} \hat{\theta}^{\mathrm{IV}}[0]-U W^{\prime} \hat{\theta}^{\prime \prime}[0]+d \hat{\theta}[0]=0, \\
U^{4} \hat{\theta}^{\mathrm{IV}}[1 / 2]-U W \hat{\theta}^{\prime \prime}[1 / 2]+d \hat{\theta}[1 / 2]=0 .
\end{array}\right.
$$

Owing to the invariance of (4.1.11) we may assume that $U=1$; then

$$
W=\frac{\hat{\theta}^{\mathrm{IV}}[0] \hat{\theta}[1 / 2]-\hat{\theta}^{\mathrm{IV}}[1 / 2] \hat{\theta}[0]}{\hat{\theta}^{\prime \prime}[0] \hat{\theta}[1 / 2]-\hat{\theta}^{\prime \prime}[1 / 2] \hat{\theta}[0]} .
$$

(1) It should be mentioned that in [23] the case $g=1$ is analysed incorrectly (the equality $\hat{\theta}^{\mathrm{IV}}[0] / \hat{\theta}[0]=\hat{\theta}^{\mathrm{IV}}[1] / \hat{\theta}[1]$ does not hold). 
The expression for $W$ can be obtained in all even simpler way by substituting in (4.1.4) for $\theta$ the odd function $\theta_{1}(z)=\theta\left[\frac{1}{2}, \frac{1}{2}\right](z)$ (the second logarithmic derivative of $\theta$ and $\theta_{1}$ differ only by a shift of the argument). Substituting $z=0$ in (4.1.4) (where $\left.\theta \mapsto \theta_{1}\right)$ we find in view of the oddness $(U=1$ ),

$$
W=-\theta_{\mathbf{1}}^{\prime \prime \prime}(0) / \theta_{\mathbf{i}}^{\prime}(0) \text {. }
$$

Before solving (4.1.6) for genera $g \geqslant 2$ we impose the following nonsingularity condition on the matrix $B_{j k}$

(4.1.14) $\operatorname{rank}\left(\hat{\theta}_{11}[n], \hat{\theta}_{12}[n], \ldots, \hat{\theta}_{g \xi}[n], \hat{\theta}[n]\right)=\frac{g(g+1)}{2}+1$.

Here the rows of the matrix in parentheses are numbered by the vector $n \in \frac{1}{2}\left(Z_{g}\right)^{2}$. Of course, non-singularity implies indecomposability (for a decomposable matrix $B=\left(\begin{array}{cc}B^{\prime} & 0 \\ 0 & B^{\prime \prime}\end{array}\right)(4.1 .14)$ has zero columns). Below (see Lemma 4.3.1) we shall show that the non-singularity condition is satisfied for the Riemann matrices of Riemann surfaces.

b) $g=2$. We rewrite (4.1.6) in the form

$$
\begin{aligned}
U_{1} W_{1} \hat{\theta}_{11}[n]+\left(U_{1} W_{2}+U_{2} W_{1}\right) & \hat{\theta}_{12}[n]+U_{2} W_{2} \hat{\theta}_{22}[n]- \\
& -d \hat{\theta}[n]=\hat{\partial}_{U}^{4} \hat{\theta}[n], \quad n \in \frac{1}{2}\left(\mathbf{Z}_{2}\right)^{2}
\end{aligned}
$$

(we recall that the subscripts denote derivatives). The non-singularity condition here is the condition for invertibility of the $(4 \times 4)$-matrix

$$
\left(\hat{\theta}_{11}[n], \hat{\theta}_{12}[n], \hat{\theta}_{22}[n], \hat{\theta}[n]\right)
$$

(the rows are indexed by the characteristic $n \in \frac{1}{2}\left(\mathbf{Z}_{2}\right)^{2}$. Let $\left(a_{n}^{11}, a_{n}^{12}, a_{n}^{22}, a_{n}\right)$ be the inverse matrix. Then from (4.1.15) we obtain

$$
\left\{\begin{array}{c}
W_{1}=\frac{1}{U_{1}} \sum_{n \in \frac{1}{2}\left(\mathbf{z}_{2}\right)^{2}} a_{n}^{11} \partial_{U}^{4} \hat{\theta}[n]=\frac{Q_{11}(U)}{U_{1}} \\
W_{2}=\frac{1}{U_{2}} \sum_{n \in \frac{1}{2}\left(z_{2}\right)^{2}} a_{n}^{22} \partial_{U}^{4} \hat{\theta}[n]=\frac{Q_{22}(U)}{U_{2}} \\
U_{1} W_{2}+U_{2} W_{1}=\sum_{n \in \frac{1}{2}\left(z_{2}\right)^{2}} a_{n}^{12} \partial_{U}^{4} \hat{\theta}[n]=Q_{12}(U)
\end{array}\right.
$$

Substituting $W_{1}$ and $W_{2}$ in the last relation, we obtain a homogeneous equation of degree 6 in $U=\left(U_{1}, U_{2}\right)$ :

$$
P\left(U_{1}, U_{2}\right)=U_{1}^{2} Q_{22}(U)-U_{1} U_{2} Q_{12}(U)+U_{2}^{2} Q_{11}(U)=0,
$$

where the polynomials $Q_{i j}(U)$ are defined in (4.1.17). From the given Riemann matrix $B_{j k}$ we obtain 6 vectors (up to a scalar factor). After this we can find $W=\left(W_{1}, W_{2}\right)$ by (4.1.17). Thus, we have proved the following result. 
Theorem 4.1.1. Let $B_{j k}$ be a Riemann $(2 \times 2)$-matrix in general position. Then the formula (4.1.2), where $\theta(z)=\theta(z \mid B)$ and the vectors $U$ and $W$ are determined from (4.1.18) and (4.1.17), gives a solution of the KdV equation (4.1.1) for any $z_{0}$.

Formulae of another type for $W$ can be obtained by using odd characteristics directly from the relation (4.1.4) (as for $g=1$ ). For example, by substituting in (4.1.4) successively $\theta \mapsto \theta[(1 / 2,0),(1 / 2,0)]$ and $\theta \rightarrow \theta[(0,1 / 2),(0,1 / 2)]$ we obtain a system of linear equations from which

$$
\begin{gathered}
\theta_{i+1}\left[\left(0, \frac{1}{2}\right),\left(0, \frac{1}{2}\right)\right] \partial_{U}^{3} \theta\left[\left(\frac{1}{2}, 0\right),\left(\frac{1}{2}, 0\right)\right]- \\
W_{i}=\frac{-\theta_{i+1}\left[\left(\frac{1}{2}, 0\right),\left(\frac{1}{2}, 0\right)\right] \partial_{U}^{3} \theta\left[\left(0, \frac{1}{2}\right),\left(0, \frac{1}{2}\right)\right]}{\theta_{1}\left[\left(\frac{1}{2}, 0\right),\left(\frac{1}{2}, 0\right)\right] \theta_{2}\left[\left(0, \frac{1}{2}\right),\left(0, \frac{1}{2}\right)\right]-} \\
-\theta_{1}\left[\left(0, \frac{1}{2}\right),\left(0, \frac{1}{2}\right)\right] \theta_{2}\left[\left(\frac{1}{2}, 0\right),\left(\frac{1}{2}, 0\right)\right]
\end{gathered}
$$

( $i=1$ or $2 ; i+$ is taken $\bmod 2$ ).

§2. The KP equation. Genus 2 and genus 3

For the KP equation

$$
\frac{3}{4} u_{y y}=\frac{\partial}{\partial x}\left(u_{t}-\frac{1}{4}\left(6 u u_{x}+u_{x x x}\right)\right)
$$

we look for a solution in the form

$$
u(x, y, t)=2 \frac{\partial^{2}}{\partial x^{2}} \log \theta\left(U x+V y+W t+z_{0}\right)
$$

(the constant $c$ in (3.1.25) vanishes under the substitution $W \mapsto W-\frac{3}{2} c U$ ). By analogy with the previous section, after substituting (4.2.2) in (4.2.1) we obtain a relation of the form

$$
\theta_{x x x x x} \theta-4 \theta_{x x x} \theta_{x}+3 \theta_{x x}^{2}+4 \theta_{x} \theta_{t}-4 \theta_{x t} \theta+3 \theta_{y y} \theta-3 \theta_{y}^{2}+8 d \theta^{2}=0
$$

(as above we denote the integration constant by $4 d$ ), where $\theta=\theta(z)$, which is valid for any $z$. The use of the addition theorem, as above, leads to the following assertion.

Lemma 4.2.1. A function of the form (4.2.2) is a solution of (4.2.1) if and only if

$$
\text { (4.2.4) } \partial_{U}^{4} \hat{\theta}[n]-\partial_{U} \partial_{W} \hat{\theta}[n]+\frac{3}{4} \partial_{V}^{2} \theta[n]+d \hat{\theta}[n]=0, \quad n \in \frac{1}{2}\left(\mathbf{Z}_{2}\right)^{g}
$$

(the notation is the same as in Lemma 4.1.1). 
The system (4.2.4) is invariant under transformations of the form

$$
\left\{\begin{array}{l}
U \mapsto \lambda U, V \mapsto \pm\left(\lambda^{2} V+2 \alpha \lambda U\right) \\
W \mapsto \lambda^{3} W+3 \lambda^{2} \alpha V+3 \lambda \alpha^{2} U, \quad d \mapsto \lambda^{4} d
\end{array}\right.
$$

Here $\lambda$ and $\alpha$ are arbitrary complex parameters, $\lambda \neq 0$. The invariance of (4.2.5) is easy to compare with that of (3.1.21).

For $g=1$ the vectors $U, V$, and $W$ are collinear (simply numbers), and the $\mathrm{KP}$ equation reduces to a $\mathrm{KdV}$ equation. Now we analyse the case $g=2$. If $U$ and $V$ are linearly dependent, then after a suitable transformation of the form (4.2.5) we find that $V=0$. In this way we again arrive at a $\mathrm{KdV}$ equation, where $U$ and $W$ for $g=2$ have been found above.

Now we assume that $U$ and $V$ are linearly independent. Then $W$ has the form

$$
W=a U+b V
$$

and (4.2.1) turns into a version of the Boussinesq equation ${ }^{(1)}$

$$
3 v_{y y}-4 a v_{x x}+4 b v_{x y}+\left(3 v^{2}+v_{x x}\right)_{x x}=0,
$$

where

$$
\begin{aligned}
& v(x+a t, y+b t)=u(x, y, t), \\
& v(x, y)=2 \frac{\partial^{2}}{\partial x^{2}} \log \theta\left(U x+V y+z_{0}\right) .
\end{aligned}
$$

Using the invariance (4.2.5) we set

$$
U_{2}=1, \quad V_{2}=0 .
$$

Solving (4.2.4) for $g=2$, by analogy with the preceding section, we obtain

$$
W_{i}=\frac{3}{4} \frac{V_{i}^{2}}{U_{i}}+\frac{Q_{i i}(U)}{U_{i}}, \quad\left(U_{2} V_{1}-U_{1} V_{2}\right)^{2}=-\frac{4}{3} P\left(U_{1}, U_{2}\right),
$$

where the polynomials $Q_{i i}$ are determined by (4.1.17) and $P$ has the form (4.1.18). Taking the "calibration" (4.2.7) into account, we obtain for $U, V$, and $W$, expressions in the parameter $z=U_{1}$

$$
\left\{\begin{array}{l}
U=(z, 1), \quad V=\left( \pm \frac{2 i}{\sqrt{3}} \sqrt{P(z, 1)}, 0\right), \\
W=\left(-z Q_{22}(z, 1)+Q_{12}(z, 1), \quad Q_{22}(z, 1)\right) .
\end{array}\right.
$$

The coefficients $a$ and $b$ of the expansion (4.2.6) have the form

$$
a=Q_{22}(z, 1), \quad b=\frac{\sqrt{3}\left(Q_{12}(z, 1)-z Q_{22}(z, 1)\right)}{2 i \sqrt{\bar{P}(z, 1)}} .
$$

Thus, we have proved the following theorem.

(1) In full agreement with the results of Ch. III, $\S 1$ : on a Riemann surface $\Gamma$ of genus 2 and a point in general position on $\Gamma$ there exists a function with a unique third-order pole at this point, therefore, we obtain the equation of a non-linear string. For the Weierstrass points (there are six of them) there exists a function with a second-order pole, and we arrive at a $\mathrm{KdV}$ equation. 
Theorem 4.2.1. Let $B=\left(B_{j k}\right)$ be any Riemann $(2 \times 2)$-matrix in general position. Then (4.2.2') solves the equation (4.2.1') for any $z_{0}$ (the coefficients $a$ and $b$ have the form (4.2.9)), where $\theta(z)=\theta(z \mid B)$, the vectors $U$ and $V$ are defined by (4.2.8), and $z$ is an arbitrary parameter $(P(z, 1) \neq 0)$.

Now we pass to genus $g=3$. Here the vectors $U, V$, and $W$ are already, generally speaking, linearly independent and the KP equation does not reduce to a $\mathrm{KdV}$ equation or to the equation of a non-linear string. To obtain the relations on $U$ we regard (4.2.4) as a linear system in 7 unknowns

$$
U_{1} W_{1}-\frac{3}{4} V_{1}^{2}, \quad U_{1} W_{2}+U_{2} W_{1}-\frac{3}{2} V_{1} V_{2}, \ldots, U_{3} W_{3}-\frac{3}{4} V_{3}^{2},-d .
$$

The matrix of this system

$$
\text { (4.2.10) } \quad\left(\hat{\theta}_{11}\lfloor n], \hat{\theta}_{12}[n], \ldots, \hat{\theta}_{33}[n], \hat{\theta}[n]\right), \quad n \in \frac{1}{2}\left(\mathbf{Z}_{2}\right)^{3},
$$

of dimension $8 \times 7$ has rank 7 by the non-singularity condition (4.1.14). The compatibility condition of this linear system has the form

(4.2.11) $R\left(U_{1}, U_{2}, U_{3}\right)=\operatorname{det}\left(\hat{\theta}_{11}[n], \hat{\theta}_{12}[n], \ldots, \hat{\theta}_{33}[n], \hat{\theta}[n], \partial_{U}^{4} \hat{\theta}[n]\right)=0$

(the characteristics $n \in \frac{1}{2}\left(Z_{2}\right)^{3}$ index the rows of this $(8 \times 8)$-matrix). It is easy to show that (4.2.11) is not identically zero for matrices $B_{j k}$ in general position (it suffices to calculate the determinant (4.2.11) in a small neighbourhood of the diagonal matrix $B$ ). Below (in $\S 3$ ) it will be shown that there must be no other relations on $U$. Let us find the vectors $V$ and $W$. Let $n_{1}, \ldots, n_{7}$ be characteristics in $\frac{1}{2}\left(\mathbf{Z}_{2}\right)^{3}$ such that the $(7 \times 7)$-minor

$$
\left(\hat{\theta}_{i j}[n], \hat{\theta}[n]\right) \quad\left(n=n_{1}, \ldots, n_{7}\right)
$$

of (4.2.10) does not vanish. Let

$$
\left(a_{n}^{i j}, a_{n}\right) \quad\left(n=n_{1}, \ldots, n_{7}\right)
$$

be the inverse matrix. Then from (4.2.4) for $g=3$ we obtain

$$
\begin{gathered}
U_{i} W_{i}-\frac{3}{4} V_{i}^{2}=Q_{i i}(U) \quad(i=1,2,3), \\
U_{i} W_{j}+U_{j} W_{i}-\frac{3}{2} V_{i} V_{j}=Q_{i j}(U) \quad(i, j=1,2,3 ; i \neq j),
\end{gathered}
$$

where the polynomials $Q_{i j}(U)$ have the form

$$
Q_{i j}{ }^{\prime}(U)=\sum_{k=1}^{7} a_{n_{k}}^{i j} \partial_{U}^{4} \hat{\theta}\left[n_{k}\right]
$$

We obtain

$$
W_{i}=\frac{3}{4} \frac{V_{i}^{2}}{U_{i}}+\frac{Q_{i i}(U)}{U_{i}} .
$$

Substituting in (4.2.15) we obtain

$$
-\frac{3}{4}\left(U_{i} V_{j}-U_{j} V_{i}\right)^{2}=\left[U_{i}^{2} Q_{j j}-U_{i} U_{j} Q_{i j}+U_{j}^{2} Q_{i i}\right]
$$


We denote by $P_{i j}=P_{i j}(U)$ the polynomial on the right-hand side of this formula. By taking the square root we obtain

$$
U_{i} V_{j}-U_{j} V_{i}=\frac{2 i}{\sqrt{3}} \sqrt{P_{i j}} \quad(i, j=1,2,3 ; i<j) .
$$

The compatibility condition for this system has the form

$$
U_{1} \sqrt{P_{23}}-U_{2} \sqrt{P_{13}}+U_{3} \sqrt{P_{12}}=0,
$$

which gives the rule for the agreement of the signs before the roots $\sqrt{P_{i j}}$. (4.2.20) is an identity and follows from (4.2.11). From (4.2.19) we determine the vector $V$ up to transformations (4.2.5):

$$
\left\{\begin{aligned}
V_{1}= & -\lambda\left(U_{3} \sqrt{P_{13}(U)}+U_{2} \sqrt{P_{12}(U)}\right) \\
V_{2} & =\lambda\left(U_{1} \sqrt{P_{12}(U)}-U_{3} \sqrt{P_{23}(U)}\right), \\
V_{3} & =\lambda\left(U_{2} \sqrt{P_{23}(U)}+U_{1} \sqrt{P_{13}(U)}\right) \\
& \lambda=\frac{2 i}{\sqrt{3}\left(U_{1}^{2}+U_{2}^{2}+U_{3}^{2}\right)} .
\end{aligned}\right.
$$

Then $W$ is determined from (4.2.17). This proves the next result.

Theorem 4.2.2. Let $B_{j k}$ be a Riemann $(3 \times 3)$-matrix in general position and $\theta$ be the corresponding $\theta$-function. Then the function $u(x, y, t)=$ $=2 \partial_{x}^{2} \log \theta\left(U x+V y+W t+z_{0}\right)$ is a solution of the KP equation (4.2.1) for any $z_{0}$, where the vectors $U, V$, and $W$ are found from (4.2.11), (4.2.21), and (4.2.17). For those vectors for which $U, V$, and $W$ are linearly dependent, the KP equation reduces to the equation of a non-linear string (3.1.39) or to the KdV equation (4.1.1), which is satisfied if and only if (4.2.11) is compatible with the system $P_{12}(U)=0, P_{13}(U)=0, P_{23}(U)=0$. This compatibility condition holds for the matrices $B_{j k}$ corresponding to hyperelliptic curves of genus 3 , and only for them.

In this theorem only the criterion for the $B_{j k}$ to be hyperelliptic remains unproved. The necessity of this criterion is obvious, since the KdV equation holds for the hyperelliptic case and $W=0$, and the vector $U$ can be found just from the system $P_{12}(U)=0, P_{13}(U)=0, P_{23}(U)=0$. This follows from (4.1.6). The sufficiency easily follows from the results of the next section.

$\S 3$. The KP equation. Genus $g \geqslant 2$. Canonical equations of Riemann surfaces

In Chapter III $U, V$, and $W$ were determined as the vectors of periods of certain meromorphic differentials on a Riemann surface $\Gamma$. Now we shall prove that the system (4.2.4) constructed from the period matrix of holomorphic differentials on $\Gamma$ has no other solutions. This will allow us to draw non-trivial consequences. 
We state without proof a number of facts that we need in what follows.

A. Let $\Gamma$ be a Riemann surface (smooth algebraic curve) of genus $g \geqslant 2$, $\omega_{1}, \ldots, \omega_{g}$ a basis of holomorphic differentials on $\Gamma$. We define the canonical mapping

$$
\Gamma \stackrel{\omega}{\rightarrow} \mathrm{C} P^{g-1} ; \quad P \mapsto\left(\omega_{1}(P): \ldots: \omega_{g}(P)\right) .
$$

Here $C P^{g-1}$ is the complex projective space of dimension $g-1$; (4.1.31) gives the mapping in homogenous coordinates. We call its image $\Gamma^{\prime}=\omega(\Gamma)$ the canonical curve and its equations the canonical equations of $\Gamma$. For a hyperelliptic curve $\Gamma$ this image is a smooth rational curve $\Gamma^{\prime}=\omega(\Gamma)$ in $\mathrm{CP}^{g-1}$ of degree $g-1$ and $\omega$ is a two-sheeted covering $\omega: \Gamma \rightarrow \Gamma^{\prime}$. For a non-hyperelliptic curve $\Gamma$ the mapping $\omega$ is a smooth imbedding (that is, $\omega: \quad \Gamma \rightarrow \Gamma^{\prime}=\omega(\Gamma)$ is an isomorphism). The degree of $\Gamma^{\prime}=\omega(\Gamma) \in \mathbf{C} P^{g-1}$ (that is, the number of points in the intersection of $\Gamma$ with any hyperplane) is $2 g-2$ (see [4]).

B. Let $(\theta) \subset J(\Gamma)$ be a theta divisor, that is, the set of zeros of a $\theta$-function (about theta divisors, see Ch. II, §4). We consider the Gauss mapping

$$
(\theta) \rightarrow \mathbf{C} P^{g-1},
$$

given for the non-singular points of the $\theta$-divisor by the formula

$$
z \mapsto \operatorname{grad} \theta(z)=\left(\theta_{1}(z): \theta_{2}(z): \ldots: \theta_{g}(z)\right) .
$$

This is of rank $g-1$ almost everywhere, that is, a covering with a branch (see [4]).(1)

C. Let $\Gamma$ be a general Riemann surface of genus $g \geqslant 5$. By $(\theta)_{\operatorname{sing}} \in J(\Gamma)$ we denote the set of singular points of the $\theta$-divisor (of those points $z$ where $\theta(z)=0$ and grad $\theta(z)=0)$. The intersection of the tangent cones of the singular points

$$
\sum_{i, j=1}^{g} x_{i} x_{j} \theta_{i j}(z)=0, \quad z \in(\theta)_{s \sin g},
$$

in $\mathbf{C} P^{g-1}$ with homogeneous coordinates $\left(x_{1}: \ldots: x_{g}\right)$ is the canonical curve $\Gamma^{\prime}$. The following exceptions are known:

a) If there is a meromorphic function $f(P)$ on $\Gamma$ with a single third-order pole at a point $Q$ (such curves are sometimes called trigonal), then the system (4.3.3) gives a ruled surface. In this case the canonical curve $\Gamma^{\prime}$ can be obtained by adding to (4.3.3) the system

$$
\text { (4.3.3') } \sum_{i, j, k} x_{i} x_{j} x_{k} \theta_{i j k}(z)=0 ; \theta(z)=\theta_{i}(z)=\theta_{i j}(z)=0 \quad(i, j=1, \ldots, g) \text {. }
$$

(1) It turns out that the image of the branch points of the Gauss mapping (4.3.2) is a surface in $\mathrm{CP}^{g-1}$, projectively dual to the canonical curve $\Gamma^{\prime}$. This observation can serve as a basis for a proof of the classical Torelli theorem, which asserts that a Riemann surface can be recovered uniquely from its Riemann matrix. To reach effective formulae from this proof one must know how to solve the transcendental equation $\theta(z)=0$. 
b) If $\Gamma$ is the smooth plane curve of degree 5 (its genus is 6), then (4.3.3) gives in $\mathbf{C} P^{5}$ a Veronese variety of the form $\left(x^{2}: x y: x z: y^{2}: y z: z^{2}\right)(x, y$, and $z$ are parameters) (see [25]).

We make a number of deductions from these assertions.

Lemma 4.3.1. Let $B=\left(B_{j k}\right)$ be the Riemann matrix of a Riemann surface $\Gamma$ of genus $g$, and let $\hat{\theta}_{i j}[n], \hat{\theta}[n]$ be the corresponding $\theta$-constants (see above, $\S 1)$. Then the following non-singularity condition holds:

$$
\operatorname{rank}\left(\hat{\theta}_{i j}[n], \hat{\theta}[n]\right)=\frac{g(g+1)}{2}+1
$$

Proof. We assume the contrary. Then there is a symmetric $(g \times g)$-matrix $\lambda_{i j}$ and a number $\lambda$ such that

$$
\sum_{i, j} \lambda_{i j} \hat{\theta}_{i j}[n]+\lambda \hat{\theta}[n]=0
$$

for any characteristic $n \in \frac{1}{2}\left(\mathbf{Z}_{2}\right)^{g}$. We multiply this by $\hat{\theta}[n](z)$ and sum over all $n \in \frac{1}{2}\left(Z_{2}\right)^{g}$. By the addition theorem (4.1.5) we obtain

$$
\sum \lambda_{i j}\left(\theta_{i j}(z) \theta(z)-\theta_{i}(z) \theta_{j}(z)\right)+\lambda \theta^{2}(z)=0
$$

In this equality we substitute for $z$ any zero of the $\theta$-function. Then $\sum_{i, j} \lambda_{i j} x_{i} x_{j}=0$, where $x_{i}=\theta_{i}(z)$ and $\theta(z)=0$. By $\mathrm{B}$ above, by changing the point $z \in(\theta)$ we can obtain any direction $\left(x_{1}, \ldots, x_{g}\right)$, therefore, $\left(\lambda_{i j}\right)$ is the null matrix. Hence, $\lambda=0$, that is, the linear combination (4.3.5) is trivial. This proves the lemma.

It has been already pointed out above that from the non-singularity condition (4.3.4) it follows that the Jacobi varieties $J(\Gamma)$ of Riemann surfaces are non-degenerate.

We return to the study of the properties of (4.2.4).

Lemma 4.3.2. For matrices $B_{i j}$ with the non-singularity condition (4.3.4) the vectors $V$ and $W$ and the constant $d$ are uniquely determined from (4.2.4) by $U$, up to transformations of the form

$$
\text { (4.3.7) } \quad V \mapsto \pm(V+2 \alpha U), \quad W \mapsto W+3 \alpha V+3 \alpha^{2} U
$$

Proof. Suppose that two sets $V, W, d$ and $\widetilde{V}, \widetilde{W}, \widetilde{d}$ correspond to a vector $U$. By subtracting the corresponding equalities (4.2.4) one from the other we obtain

$$
\sum_{i j}\left[\frac{3}{4}\left(V_{i} V_{j}-\widetilde{V}_{i} \widetilde{V}_{j}\right)-U_{i}\left(W_{j}-\widetilde{W}_{j}\right)\right] \hat{\theta}_{i j}[n]+(d-\tilde{d}) \hat{\theta}[n]=0
$$


It follows from (4.3.4) that all the coefficients of $\hat{\theta}_{i j}[n](i \leqslant j)$ and $\hat{\theta}[n]$ are zero:

$$
\left\{\begin{array}{l}
\frac{3}{4}\left(V_{i}^{2}-\widetilde{V}_{i}^{2}\right)-U_{i}\left(W_{i}-\widetilde{W}_{i}\right)=0 \\
\frac{3}{2}\left(V_{i} V_{j}-\widetilde{V}_{i} \widetilde{V}_{j}\right)-U_{i}\left(W_{j}-\widetilde{W}_{j}\right)-U_{j}\left(W_{i}-\tilde{W}_{i}\right)=0 \\
d=\tilde{d}
\end{array}\right.
$$

By eliminating $W_{i}-\widetilde{W}_{i}$ from the first line and substituting $W_{i}-\widetilde{W}_{i}$ and $W_{j}-\widetilde{w}_{j}$ in the second we obtain

or

$$
\left(U_{i} V_{j}-\dot{U}_{j} V_{i}\right)^{2}=\left(U_{i} \tilde{V}_{j}-U_{j} \tilde{V}_{i}\right)^{2},
$$

$$
U_{i}\left(V_{j} \pm \tilde{V}_{j}\right)-U_{j}\left(V_{i} \pm \tilde{V}_{i}\right)=0,
$$

hence $\widetilde{V}= \pm(V+2 \alpha U)$. This proves the lemma.

Let us now study what kind of vectors $U$ are possible. We recall from Chapter III the construction of the exact solutions of a KP equation.

According to this construction the vector $U=\left(U_{1}, \ldots, U_{g}\right)$ in the solution formula (4.2.2) depends on the point $Q$ of $\Gamma$ as on a parameter, $U=U(Q)$, and was constructed as the vector of $b$-periods of the normalized differential $\Omega_{Q}$ of the second kind with a double pole at $Q$. Lemma 2.1 .2 (see (2.1.21)) implies that

$$
\left(U_{1}(Q): \ldots: U_{g}(Q)\right)=\left(\omega_{1}(Q): \ldots: \omega_{g}(Q)\right) .
$$

In other words, $Q \mapsto U(Q)$ is the canonical mapping (4.3.1). Thus, the set of vectors $U$ that are solutions of (4.2.4) contains the canonical curve $\Gamma^{\prime}=\omega(\Gamma)$. We claim that the system (4.2.4) has no other solutions $U$. We first consider the case $g=2$ or 3 .

Theorem 4.3.1. For $g=2$ or 3 any Riemann matrix $B_{j k}$ satisfying the nonsingularity condition (4.3.4) is a period matrix of holomorphic differentials on some Riemann surface $\Gamma$. For $g=2$ this Riemann surface is given by the equation

$$
w^{2}=P(z, 1),
$$

where the polynomial $P\left(U_{1}, U_{2}\right)$ is determined by (4.1.18). For $g=3 a$ non-hyperelliptic surface $\Gamma$ is given in $\mathrm{CP}^{2}$ by a homogeneous equation of degree 4

$$
R(U)=\operatorname{det}\left(\hat{\theta}_{i j}[n], \quad \hat{\theta}[n], \partial_{U}^{4} \hat{\theta}[n]\right)=0 .
$$

The hyperelliptic case of genus 3 is distinguished by (4.3.11) together with the system

$$
P_{12}(U)=0, \quad P_{13}(U)=0, \quad P_{23}(U)=0,
$$

where the polynomials $P_{i j}(U)$ are of the form (4.2.18). In this case the curve (4.3.11) is rational $(R(U)$ is a complete square) and the required Riemann surface $\Gamma$ is a two-sheeted covering of it and the branch points are the solution of the systems (4.3.11), (4.3.12). 
Proof. Suppose first that $B_{j k}$ is a period matrix of holomorphic differentials on a Riemann surface $\Gamma$. Then the set of vectors $U$ solving the systems (4.2.4) contains the canonical curve $\Gamma^{\prime}$. For $g=2, \Gamma^{\prime}=\mathbf{C P} P^{1}$, and to the six Weierstrass points on $\Gamma$ there correspond the points $\left(U_{1}: U_{2}\right) \in \Gamma^{\prime}$ for which $V=0$ (and the KP equation reduces to a $\mathrm{KdV}$ equation, by the results of Chapter III). These six points are precisely the solutions of the equation $P\left(U_{1}, U_{2}\right)=0$, which implies (4.3.10).

For $g=3$ the canonical curve $\Gamma^{\prime}=\omega(\Gamma)$ is a curve of degree 4 , which must therefore be given in $\mathbf{C} P^{2}$ by (4.3.11). For a hyperelliptic curve $\Gamma$ its eight Weierstrass points go over into the points $U \in \Gamma^{\prime}$ for which $V=0$. By the calculations of the preceding section, these points $U$ on $\Gamma^{\prime}$ can be found from (4.3.12). Conversely, if the system (4.3.11), (4.3.12) is compatible, then $V=0$. By Lemma 4.3.2 and the construction in Chapter III, $V$ is the vector of $b$-periods of a normalized differential $\Omega$ of the second kind with only one third-order pole. If $V=0$, then $z=\int \Omega$ is a single-valued function on $\Gamma$ with only one second-order pole. Such a function gives a two-sheeted covering $\Gamma \rightarrow \mathbf{C} P^{1}$, which means that $\Gamma$ is hyperelliptic.

Thus, we have proved that the mapping

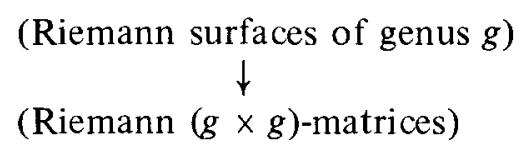

is an imbedding for $g=2$ and 3 . But the dimensions of these spaces are identical: $3 g-3=g(g+1) / 2$ for $g=2$ and 3; moreover, both are irreducible. Thus, (4.3.13) is an isomorphism (almost everywhere). This proves the theorem.

As a consequence we obtain the assertion used in $\S 1$ and $\S 2$ : for $g=2$ and a KP equation the vector $U=\left(U_{1}, U_{2}\right)$ is arbitrary; for $g=3$ and a KP equation all the relations on $U$ are given by (4.3.11).

By Lemma 4.3.2, a projection is defined of the set of non-zero solutions of (4.2.4) into the space $\mathbf{C} P^{-1}$ with homogeneous coordinates $\left(U_{1}: \ldots: U_{g}\right)$,

$$
(U, V, W, d) \mapsto U
$$

This projection is one-to-one up to transformations of the form (4.3.7).

Theorem 4.3.2. Let $B_{j k}$ be a period matrix of holomorphic differentials on a Riemann surface $\Gamma$ of genus $g \geqslant 2$. Then for $\Gamma$ in general position the image of the projection (4.3.14) of the solutions of the corresponding system (4.2.4) in the space $\mathbf{C P g}^{g-1}$ is the canonical curve $\Gamma^{\prime}=\omega(\Gamma)$.

In other words, by eliminating the variables $V, W$, and $d$ from (4.2.4) we obtain the equations of the canonical curve $\Gamma^{\prime}$. It is useful to mention that to a change of $B_{j k}$ into an equivalent matrix (in the sense of Ch. I, §3) there corresponds a projective transformation of $\Gamma^{\prime}$ in $\mathbf{C} P_{U}^{g-1}$. 
Proof of the theorem. For $g=2$ and 3 everything is proved in Theorem 4.3.2. For $g \geqslant 4$ we substitute any point $z \in(\theta)_{\operatorname{sing}}$ in (4.2.3), which is equivalent to the system (4.2.4). Of (4.2.3) only one term remains:

(4.3.15) $\sum_{i, j} U_{i} U_{j} \theta_{i j}(z)=0 ; \quad \theta(z)=0, \theta_{i}(z)=0 \quad(i=1, \ldots, g)$.

We consider four cases.

a) $\Gamma$ is a general curve of genus $g \geqslant 5$. In this case the system (4.3.15) cuts out precisely the canonical curve, according to $\mathrm{C}$.

b) $\Gamma$ is a hyperelliptic curve. Then the system (4.3.15) gives a rational curve $\Gamma^{\prime}=\omega(\Gamma)$. The images of the Weierstrass points on $\Gamma^{\prime}$ can be found from (4.2.4) together with the system $V_{1}=\ldots=V_{g}=0$.

c) $\Gamma$ is a trigonal curve. We differentiate (4.2.3) with respect to $z_{i}(i=1, \ldots, g)$ and substitute in the resulting expression a singular point of the theta divisor $(\theta(z)=0$, grad $\theta(z)=0)$. After cancelling $\theta_{x i}(z)$ we obtain

$$
\sum_{i, j, k} U_{i} U_{j} U_{k} \theta_{i j k}(z)=0,
$$

which together with (4.3.15) gives the canonical curve $\Gamma^{\prime}$.

d) The case when $\Gamma$ is a plane curve of degree 5 is analysed similarly.

The theorem is now proved.

Another proof (suitable for all special cases, including genus $g=4$ ) can be obtained by the methods of the following section.

Theorem 4.3.2 gives a new proof of Torelli's theorem (see the footnote at the beginning of this section), which is more effective, since only algebraic operations (there is no need to solve the transcendental equation $\theta(z)=0$ ) are required to recover the canonical equation of an algebraic curve from its Riemann matrix $B_{j k}$.

\$4. The problem of Riemann on relations between the periods of holomorphic differentials on a Riemann surface and the conjecture of Novikov

According to the conjecture of Novikov, the compatibility of the system (4.2.4) gives a set of relations on the matrix $B_{j k}$ that is necessary and sufficient for $B_{j k}$ to be the period matrix of some Riemann surface. Here we sketch a proof of this conjecture for one of the components of the variety of such matrices $B_{j k}$, for which the system (2.4.2) is compatible. The question of whether or not there are other components remains open.

To give a precise statement of the principal result we introduce the following objects. Let $M_{g}$ be a variety parametrizing the Riemann surfaces of genus $g$ (the "variety of modules" of Riemann surfaces). This is irreducible and its complex dimension is $3 g-3$ for $g \geqslant 2$. The period mapping

$$
M_{g} \rightarrow H_{g} / \Lambda_{g}
$$


where $H_{g}$ is the set of all Riemann $(g \times g)$-matrices (the Siegel half-plane) and $\Lambda_{g}=\operatorname{Sp}(g, Z) /\{ \pm 1\}$ (see Ch. I, §3), associates with every Riemann surface its period matrix of holomorphic differentials.

By $\widetilde{M}_{g}$ we denote the natural fibering over $M_{g}$, where the fibre over a given point is the corresponding curve. The dimension of $\widetilde{M}_{g}$ is $3 g-2$. The period mapping (4.4.1) extends trivially to a mapping

$$
\tilde{M}_{g} \rightarrow H_{g} / \Lambda_{g \bullet}
$$

Let $N_{g}$ be its graph. Now we introduce another variety $X_{g}$, whose points are the sets $(U, V, W, d, B)$, where $U, V, W \in \mathbf{C}^{g}, d \in \mathbf{C}$, and $B \in H_{g}$, factored by the action of the following groups:

$$
\left\{\begin{array}{l}
U \mapsto \lambda U, \quad V \mapsto \pm\left(\lambda^{2} V+2 \alpha \lambda U\right), \\
W \mapsto \lambda^{3} W+3 \lambda^{2} \alpha V+3 \lambda \alpha^{2} U . \quad d: \lambda^{4} d, \quad B \mapsto B
\end{array}\right.
$$

$(\lambda, \alpha \in \mathbf{C}, \lambda \neq 0)$, and

$$
\text { (4.4.4) }\left\{\begin{array}{l}
B^{\prime}=2 \pi i(\alpha B+2 \pi i \beta)(\gamma B+2 \pi i \delta)^{-1}, \\
U^{\prime}=2 \pi i M^{-1} U, \text { where } M=\gamma B+2 \pi i \delta, \\
V^{\prime}=2 \pi i M^{-1} V, \\
W^{\prime}=2 \pi i M^{-1} W+\frac{2 \pi i}{3} M^{-1} U\{U, U\}, \text { where }\{X, Y\}=X^{t} M^{-1} \gamma Y, \\
d^{\prime}=d+\frac{3}{8}\{V, V\}-\frac{1}{2}\{U . W\}-\frac{3}{4}\{U, U\}^{2} .
\end{array}\right.
$$

Here $\left(\begin{array}{ll}\alpha & \beta \\ \gamma & \delta\end{array}\right) \in \operatorname{Sp}(g, \mathbf{Z})$.

Theorem 4.4.1. The system (4.2.4) specifies on $X_{g}$ an algebraic variety one of whose irreducible components coincides with the graph $N_{g}$ of the period mapping (4.4.2).

By projecting this component on $H_{g} / \Lambda_{g}$, that is, eliminating the variables $U, V, W, d$ from (4.2.4), we obtain a full set of relations between the periods of the holomorphic differentials on the Riemann surfaces.

Idea of the Proof. It is easy to verify that the set of zeros $Y_{g}$ of (4.2.4) is invariant under the transformations $(4.4 .3),(4.4 .4)$, therefore $Y_{g}$ is an algebraic subvariety of $X_{g}$. It is clear that $Y_{g}$ contains $N_{g}$. Consequently, it is sufficient to calculate the dimension of the component $\hat{N}_{g} \subset Y_{g}$, that contains $N_{g}$.

To do this it is sufficient to show that at a general point of $\hat{N}_{g}$ the variables $d$ and $B$ in (4.2.4) can be expressed uniquely by the variables $U, V, W$ with the equivalence relation (4.4.3). For the group (4.4.3) is twodimensional and among the parameters exactly $3 g-2$ are independent. It suffices to prove this for matrices $B$ whose diagonal elements $B_{i i}$ tend to $-\infty$. Such matrices correspond to rational curves with $g$ double points. In this case the system (4.2.4) can be solved explicitly by "perturbation theory" as 
a power series in $\epsilon_{i}=\exp B_{i i}$, and any term of the series can be written out in effective formulae. From this the assertion on the dimension of $\hat{N}_{g}$ follows easily, and this completes the proof.

Theorem 4.3.2 can also be proved by "perturbation theory", including the special cases listed in $\S 3$. For the detailed calculations and the proof of Theorem 4.4.1 see [45].

In conclusion of this chapter we mention that the methods developed here are also applicable to other non-linear equations that can be integrated in terms of $\theta$-functions.

\section{Chapter $V$}

\section{EXAMPLES OF HAMILTONIAN SYSTEMS THAT ARE INTEGRABLE IN TERMS OF TWO-DIMENSIONAL THETA FUNCTIONS}

\section{$\S 1$. Two-zone potentials}

According to the scheme of Chapter III, a hyperelliptic curve $\Gamma$ of genus 2 of the form

$$
w^{2}=P_{5}(z), P_{5}(z)=\left(z-z_{1}\right) \ldots\left(z-z_{5}\right)
$$

and a Weierstrass point $z=\infty$ on it give rise to a pair of commuting operators $L, A$

$$
[L, A]=0
$$

where

$$
\begin{gathered}
L=d^{2} / d x^{2}+u, \\
A=16 \frac{d^{5}}{d x^{5}}+20\left(u \frac{d^{3}}{d x^{3}}+\frac{d^{3}}{d x^{3}} u\right)+30 u \frac{d}{d x} u- \\
-5\left(u^{\prime \prime} \frac{d}{d x}+\frac{d}{d x} u^{\prime \prime}\right)+c_{1}\left[4 \frac{d^{3}}{d x^{3}}+3\left(u \frac{d}{d x}+\frac{d}{d x} u\right)\right]+c_{2} \frac{d}{d x} .
\end{gathered}
$$

The commutativity equation (5.1.2) on $u=u(x)$ (one of the important examples of the Novikov equations) can be written in the Lagrange form

$$
\delta\left(\int \Lambda d x\right) / \delta u(x)=0
$$

with the Lagrangian

(5.1.6) $\Lambda=\Lambda\left(u, u^{\prime}, u^{\prime \prime}\right)=\frac{u^{\prime \prime 2}}{2}-\frac{5}{2} u^{\prime \prime} u^{2}+\frac{5}{2} u^{4}+$

$$
+c_{1}\left(\frac{u^{\prime 2}}{2}+u^{3}\right)+c_{2} u^{2}+c_{3} u
$$

$\left(c_{1}, c_{2}, c_{3}\right.$ are constants, $\delta / \delta u(x)$ is the variational derivative). According to the theory of variational problems with higher derivatives (see [13]), (5.1.5) is equivalent to a Hamiltonian system with two degrees of freedom and the Hamiltonian

$$
H=p_{1} p_{2}+V\left(q_{1}, q_{2}\right) .
$$


Here

$$
\begin{aligned}
& q_{1}=u, \quad q_{2}=u^{\prime \prime}-5 u^{2}, \quad p_{1}=q_{2}^{\prime}, \quad p_{2}=u^{\prime}, \\
& V=-\frac{q_{2}^{2}}{2}-\frac{5}{2} q_{2} q_{1}^{2}-\frac{5}{8} q_{1}^{4}+\frac{c_{2}}{2} q_{1}^{2}+c_{3} q_{1},
\end{aligned}
$$

and under the change $u \mapsto u+$ const the constant $c_{1}$ vanishes. The system (5.1.7) is completely integrable; its integrals in involution have the form $J_{1}=H$,

$$
\begin{gathered}
J_{2}=p_{1}^{2}+2 q_{1} p_{1} p_{2}+\left(2 q_{2}-c_{2}\right) p_{2}^{2}+D\left(q_{1}, q_{2}\right), \\
D=q_{1}^{5}+c_{2} q_{1}^{3}-4 q_{1} q_{2}^{2}+2 c_{2} q_{1} q_{2}+2 c_{3} q_{2} .
\end{gathered}
$$

The explicit coordinates $\gamma_{1}$ and $\gamma_{2}$ on the level surfaces $J_{1}=$ const and $J_{2}=$ const have the form

(5.1.12) $\quad \gamma_{1}+\gamma_{2}=\frac{u}{2}, \quad \gamma_{1} \gamma_{2}=\frac{1}{8}\left(3 u^{2}+u^{\prime \prime}\right)+\frac{1}{2} \sum_{i<j} z_{i} z_{j}$. Here $z_{1}, \ldots, z_{5}$ are the zeros of $P_{5}(z)$ of the form

$$
P_{5}(z)=z^{5}+\frac{1}{8} c_{2} z^{3}+\frac{1}{16} c_{3} z^{2}+\left(\frac{1}{32} J_{1}+\frac{1}{16} c_{2}^{2}\right) z+\frac{J_{2}-c_{2} c_{3}}{2^{8}} .
$$

In these variables the system (5.1.5) can be rewritten in the form

$$
\gamma_{1}^{\prime}=\frac{2 i \sqrt{P_{5}\left(\gamma_{1}\right)}}{\gamma_{1}-\gamma_{2}}, \quad \gamma_{2}^{\prime}=\frac{2 i \sqrt{P_{5}\left(\gamma_{2}\right)}}{\gamma_{2}-\gamma_{1}} .
$$

The system (5.1.14) coincides, up to the factor $2 i$, with (2.4.55), which arose in the Jacobi inversion theory. This system can be integrated by an Abel transformation, and the explicit solution of (5.1.15), according to Chapter III, has the form

$$
u(x)=2 \frac{\partial^{2}}{\partial x^{2}} \log \theta\left(U x+z_{0}\right)+c .
$$

Here the Riemann $\theta$-function is constructed from the Riemann surface (5.1.1) (where $P_{5}(z)$ has the form (5.1.13) and $U$ is the vector of $b$-periods of a normalized differential $\Omega$ of the second kind with a pole at $z=\infty$ and principal part of the form $d(\sqrt{z})$ :

$$
\left(5.1 .15^{\prime}\right) \quad \Omega=\frac{z^{2}+a z+b}{2 \sqrt{P_{5}(z)}} d z
$$

$\oint_{a_{1}} \Omega=\oint_{a_{3}} \Omega=0 ; U_{i}=\oint_{b_{i}} \Omega(i=1,2)$.

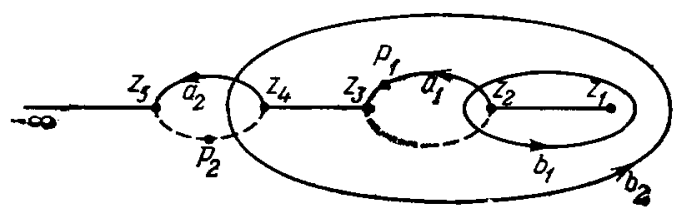

Fig. 4. Spectrum of a two-zone potential. $a_{1}, a_{2}, b_{1}, b_{2}$ are the basis cycles on the Riemann surface.

The solutions are "enumerated" by an arbitrary two-dimensional vector $z_{0}$ :

$$
z_{0}=-A\left(P_{1}, P_{2}\right)-K
$$

(see Ch. III, $\S 1$ ); $P_{1}$ and $P_{2}$ are points on $\Gamma$ (non-special divisor of degree 2 ). 
Suppose that the roots $z_{1}>\ldots>z_{5}$ of $P_{5}(z)$ are real (and distinct). Suppose further, that the points $P_{1}$ and $P_{2}$, which specify the solution $u$, have the form

$$
P_{1}=\left(\gamma_{1}, \sqrt{P_{5}\left(\gamma_{1}\right)}\right), \quad P_{2}=\left(\gamma_{2}, \sqrt{P_{5}\left(\gamma_{2}\right)}\right),
$$

where $\gamma_{1}$ and $\gamma_{2}$ are real numbers, and

$$
z_{3} \leqslant \gamma_{1} \leqslant z_{2}, \quad z_{5} \leqslant \gamma_{2} \leqslant z_{4} .
$$

Then $u(x)$ is almost periodic with two unknown periods. The spectrum of the operator $L=d^{2} / d x^{2}+u(x)$ in $\mathscr{L}_{2}(-\infty, \infty)$ is a ray $\left(-\infty, z_{1}\right]$ with two gaps $\left(z_{5}, z_{4}\right)$ and $\left(z_{3}, z_{2}\right)$ (Fig. 4). This means that $u(x)$ is a "two-zone potential". The condition (5.1.17) means that the points $P_{1}$ and $P_{2}$ on $\Gamma$ lie on the cycles over the gaps. The eigenfunction $\psi$ of $L$,

$$
L \psi=z \psi,
$$

is meromorphic on $\Gamma \backslash \infty$, has poles at $P_{1}$ and $P_{2}$, and exponential asymptotic behaviour as $P \rightarrow \infty$, that is, it is a Baker-Akhiezer function of the form

$$
\psi(x, P)=\exp \left(x \int_{\infty}^{P} \Omega\right) \frac{\theta\left(A(P)+x U+z_{0}\right) \theta\left(z_{0}\right)}{\theta\left(A(P)+z_{0}\right) \theta\left(x U+z_{0}\right)} .
$$

Let us explain this notation: $\mathcal{X}$ is the principal value of the integral; $A(P)$ is the Abel mapping, and

$$
A(P)=\left(A_{1}(P), A_{2}(P)\right)=\left(\int_{\infty}^{P} \omega_{1}, \int_{\infty}^{; P} \omega_{2}\right),
$$

where

$$
\omega_{1}=\frac{a_{1} z+b_{1}}{\sqrt{P_{5}(z)}} d z, \quad \omega_{2}=\frac{a_{2} z+b_{2}}{\sqrt{P_{B}(z)}} d z .
$$

is a normalized basis of holomorphic differentials on $\Gamma$. The eigenfunction (5.1.19) of $L$ is the Bloch function: the group of periods of the logarithmic derivative $\psi^{\prime} / \psi$ is the same as that of the potential $u(x)$. Thus, in this case $u(x)$ has the right analytic properties. In this case $\Gamma$ is called the spectrum of $L$.

Of course, hyperelliptic surfaces of genus $g \geqslant 2$ lead to finite-zone operators with $g$ gaps in the spectrum. The case $g=2$ stands out for its effectiveness. By Ch. IV,$\S 1$, a two-zone potential can be constructed by means of the following elementary operations:

1) We take any (indecomposable) Riemann matrix

$$
B=\left(\begin{array}{ll}
B_{11} & B_{12} \\
B_{21} & B_{22}
\end{array}\right)
$$

and construct from it the $\theta$-function $\theta\left(z_{1}, z_{2}\right)$ by (1.1.1). 
2) We take any solution $U=\left(U_{1}, U_{2}\right)$ of (4.1.18). Then

$$
u(x)=2 \partial^{2} / \partial x^{2} \log \theta\left(x U+z_{0}\right)
$$

is a two-zone potential ( $z_{0}$ is an arbitrary vector). Its spectrum (the Riemann surface $\Gamma$ ) is given by (4.3.10). All two-zone potentials are obtained in this way.

\section{$\S 2$. The problem of Sophie Kovalevskaya}

In the case treated by Sophie Kovalevskaya the equations of motion of a heavy solid with a fixed point have the form

$$
\begin{cases}2 \dot{p}=g r, & \dot{\gamma}_{1}=r \gamma_{2}-q \gamma_{3}, \\ 2 \dot{q}=-p r-\mu \gamma_{3}, & \dot{\gamma}_{2}=p \gamma_{3}-r \gamma_{1}, \\ \dot{r}=\mu \gamma_{2}, & \dot{\gamma}_{3}=q \gamma_{1}-p \gamma_{2}\end{cases}
$$

$(\mu=$ const $)$. These equations have the following integrals:

$$
\left\{\begin{array}{l}
H=2\left(p^{2}+q^{2}\right)+r^{2}-2 \mu \gamma_{1} \quad \text { (energy) } \\
L=2\left(p \gamma_{1}+q \gamma_{2}\right)+r \gamma_{3} \quad \text { (impulse moment) } \\
K=\left(p^{2}-q^{2}+\mu \gamma_{1}\right)^{2}+\left(2 p q+\mu \gamma_{2}\right)^{2} \quad \text { (Kovalevskaya integral). }
\end{array}\right.
$$

Moreover, there is the constraint

$$
\gamma_{1}^{2}+\gamma_{2}^{2}+\gamma_{3}^{2}=1 \text {. }
$$

We consider a joint level surface of these integrals

$$
H=6 h, \quad L=2 l, \quad K=k^{2},
$$

where $H, l$, and $k^{2}$ are constants. Under the constraint (5.2.3) these equations specify a two-dimensional surface (the invariant variety of the dynamic system (5.2.1)). We introduce coordinates $s_{1}$ and $s_{2}$ on this surface (the Kovalevskaya variables), by setting

$$
s_{1,2}=3 h+\frac{R\left(x_{1}, x_{2}\right) \mp \sqrt{R\left(x_{1}\right) R\left(x_{2}\right)}}{\left(x_{1}-x_{2}\right)^{2}},
$$

where $x_{1,2}=p \pm i q, R(z)=-z^{4}+6 h z^{2}+4 \mu l z+\mu^{2}-k^{2}$,

(5.2.6) $R\left(x_{1}, x_{2}\right)=-x_{1}^{2} x_{2}^{2}+6 h x_{1} x_{2}+2 \mu l\left(x_{1}+x_{2}\right)+\mu^{2}-k^{2}$.

An easy calculation shows that (5.2.1) can be written as follows in the variables $s_{1}$ and $s_{2}$ :

$$
\dot{s}_{1}=\frac{i \sqrt{P_{5}\left(s_{1}\right)}}{2\left(s_{1}-s_{2}\right)}, \quad \dot{s}_{2}=\frac{i \sqrt{P_{5}\left(s_{2}\right)}}{2\left(s_{2}-s_{1}\right)},
$$

where $P_{5}(z)$ is a polynomial of degree 5 of the form

$$
p_{5}(z)=\left\{z\left[(z-3 h)^{2}+\mu^{2}-k^{2}\right]-2 \mu^{2} l^{2}\right\}\left((z-3 h)^{2}-k^{2}\right) .
$$


The equations (5.2.7) coincide (up to a factor) with the commutativity equation (5.1.2), in $\S 1$ on the level surface of two integrals. These equations can be integrated by the Abel transformation $\Gamma \rightarrow J(\Gamma)$, where the Riemann surface $\Gamma$ of genus 2 is given by an equation

$$
w^{2}=P_{5}(z) \text {. }
$$

An expression of the Kovalevskaya variables in terms of the $\theta$-functions on the torus $T^{4}=J(\Gamma)$ can be deduced from Ch. II, §4. An expression of the original variables $p, q, r, \gamma_{1}, \gamma_{2}, \gamma_{3}$ in terms of the Kovalevskaya variables can be found in the book $[10]$.

§3. The problems of Neumann and Jacobi. The general system of Garnier

In the Neumann problem on the motion of particles on a two-dimensional sphere

$$
x^{2}=\sum_{i=1}^{3} x_{i}^{2}=1
$$

under the action of the quadratic potential

$$
U(x)=\frac{1}{2} \sum_{i=1}^{3} a_{i} x_{i}^{2}, \quad a_{i}=\text { const }
$$

the equations of motion have the form

$$
\begin{gathered}
\ddot{x}_{i}=-a_{i} x_{i}+\lambda(t) x_{i} \quad(i=1,2,3), \\
x^{2}=\sum_{i=1}^{3} x_{i}^{2}=1,
\end{gathered}
$$

where $\lambda(t)$ is the Lagrange multiplier arising from imposing the constraint (5.3.1). The system (5.3.3), (5.3.3') can be obtained from the Hamiltonian flow on $\mathbf{R}^{e}$ with the Hamiltonian

$$
H=\frac{1}{2} \sum_{i=1}^{3} a_{i} x_{i}^{2}+\frac{1}{2}\left(x^{2} y^{2}-(x y)^{2}\right)
$$

by restriction to the surface $x^{2}=1$ (here $x y=\sum x_{i} y_{i}$ ). The functions

$$
F_{k}(x, y)=x_{k}^{2}+\sum_{i \neq k} \frac{\left(x_{k} y_{i}-x_{i} y_{k}\right)^{2}}{a_{i}-a_{k}} \quad(k=1,2,3)
$$

are a system of independent integrals in involution for the system with the Hamiltonian (5.3.4). $H$ itself has the form

The transformation

$$
H=\frac{1}{2} \sum_{i=1}^{3} a_{i} F_{i} .
$$

$$
x^{!}=y, y^{\iota}=-x_{i} \quad H^{\prime}=\sum_{i=1}^{3} a_{i}^{-1} F_{i}
$$


takes the thus constructed Hamiltonian flow into a geodesic flow on a threeaxis ellipsoid (for positive $a_{i}$ )

$$
\sum_{i=1}^{3} \frac{x_{i}^{2}}{a_{i}}=1
$$

The problem of geodesics on a three-axis ellipsoid is called the Jacobi problem.

We shall show that the Neumann problem (hence, also the Jacobi problem) can be integrated in terms of $\theta$-functions of genus 2 . Following [28] and [29], we reduce the Neumann problem to the problem on twozone potentials considered in $\S 1$.

Let $\psi_{1}, \psi_{2}$, and $\psi_{3}$ be the eigenfunctions of the operator $L=d^{2} / d x^{2}+u(x)$ with the eigenvalues $-a_{1},-a_{2}$, and $-a_{3}$, respectively, that is, solutions of the equations

$$
L \psi_{i}=-a_{i} \psi_{i}(i=1,2,3) .
$$

These equations can be rewritten in the form

$$
\psi_{i}^{\prime \prime}=-a_{i} \psi_{i}-u(x) \psi_{i}
$$

which are the same as the equations (5.3.3) of the Neumann problem, after the change $x \rightarrow t, \psi_{i} \rightarrow x_{i},-u(x) \rightarrow \lambda(t)(\lambda$ is the Lagrange multiplier). It remains to satisfy the constraint equation $\sum x_{i}^{2}=1$. For this purpose we choose the potential $u(x)$ to be two-zoned and so that the numbers $-a_{1},-a_{2}$, and $-a_{3}$ are at the ends of the zones of the spectrum (see Fig. 4), one for each zone. For example, we take the ends of the zones:

$$
z_{5}=-a_{3}<z_{4}<z_{3}=-a_{2}<z_{2}<z_{1}=-a_{1}
$$

where the Riemann surface $\Gamma$ (the spectrum of $L$ ) has the form

$$
w^{2}=P_{5}(z), \quad P_{5}(z)=\prod_{i=1}^{5}\left(z-z_{i}\right) \text {. }
$$

We choose the solutions $\psi_{1}, \psi_{2}$, and $\psi_{3}$ of (5.3.9) as follows: let $\psi(x, P)$ be the Bloch eigenfunction of $L$, which is meromorphic on (5.3.12) (the BakerAkhiezer function). We set

where

$$
\psi_{i}(x)=\alpha_{i} \psi\left(x,-a_{i}\right)
$$

$$
\alpha_{i}=\left[\prod_{j=i}\left(a_{i}-a_{j}\right)\right]^{-1 / 2} .
$$

Then we have a simple proposition.

Proposition. The functions $\psi_{1}, \psi_{2}$, and $\psi_{3}$ of the form (5.3.14), (5.3.13) satisfy the equation

$$
\psi_{1}^{2}+\psi_{2}^{2}+\psi_{3}^{2} \equiv 1
$$

A proof is in [29]. 
From the formula (5.1.19) for the Bloch function $\psi(x, P)$ and from (5.3.13) we immediately obtain the form of the general solution of the Neumann problem. For example, for the ends of the zones (5.3.11) and the basis of cycles illustrated in Fig. 4 we obtain the solutions:

$$
\begin{aligned}
x_{1}(t) & =\alpha_{1} \frac{\theta[(0,1 / 2),(0,0)]\left(t U+z_{0}\right) \theta\left(z_{0}\right)}{\theta[(0,1 / 2),(0,0)]\left(z_{0}\right) \theta\left(t U+z_{0}\right)}, \\
x_{2}(t) & =\alpha_{2} \frac{\theta[(1 / 2,0),(0,1 / 2)]\left(t U+z_{0}\right) \theta\left(z_{0}\right)}{\theta[(1 / 2,0),(0,1 / 2)]\left(z_{0}\right) \theta\left(t U+z_{0}\right)}, \\
x_{3}(t) & =\alpha_{3} \frac{\theta[(0,0),(1 / 2,1 / 2)]\left(t I I+z_{0}\right) \theta\left(z_{0}\right)}{\theta[(0,0),(1 / 2,1 / 2)]\left(z_{0}\right) \theta\left(t U+z_{0}\right)} .
\end{aligned}
$$

Here the constants $\alpha_{1}, \alpha_{2}$, and $\alpha_{3}$ have the form (5.3.14); the $\theta$-functions are constructed from the Riemann surface (5.3.12); $z_{0}$ is an arbitrary twodimensional vector (a point of the Jacobi variety $J(\Gamma)$ ); the vector $U$ is the same as in $\S 1$. To obtain real solutions $z_{0}$ must have the form (5.1.16)(5.1.17). The Jacobi problem can now be integrated after using (5.3.7).

The systems of Neumann and Jacobi with two degrees of freedom, which we have discussed in detail, can be rewritten almost automatically for higher dimensions. The integration of these systems can always be reduced to finite-zone potentials (see [28], [29]).

The Neumann system can also be obtained from a more general integrable system, discovered by Garnier [40],

$$
\left\{\begin{array}{l}
x_{i}^{\prime \prime}=x_{i}\left(\sum x_{j} y_{j}+a_{i}\right), \\
y_{i}^{\prime \prime}=y_{i}\left(\sum y_{j} x_{j}+a_{i}\right) \quad(i=1, \ldots, n) .
\end{array}\right.
$$

The Neumann system on a sphere can be obtained straight away on the invariant plane $x_{i}=a_{i} y_{i}$. Another interesting case is the system of anharmonic oscillators, which is obtained from (5.3.16) by restriction to the plane $x_{i}=y_{i}$. The system of Garnier is equivalent (under a suitable choice of the parameter $\tau$ ) to the commutation conditions

$$
d A(\lambda) / d \tau=[A(\alpha), A(\lambda)] /(\lambda-\alpha),
$$

where the matrix $A=\left(A_{i j}\right)$ has the form

$$
\left\{\begin{array}{lrl}
A_{11}=\lambda^{2}-\sum x_{i} y_{i} ; & \\
A_{1 i}=x_{i-1} \lambda+x_{i-i}^{\prime} ; & A_{i 1}=y_{i-1} \lambda-y_{i-1}^{\prime} \\
A_{i j}=x_{i-1} y_{i-1}-a_{i-1} \delta_{i j} & (i, j=2, \ldots, n+1) .
\end{array}\right.
$$

This system can be integrated in terms of the $\theta$-functions of a Riemann surface of the form

$$
R(\lambda, \mu) \equiv \operatorname{det}(A(\lambda)-\mu \cdot 1)=0 .
$$


\$4. Movement of a solid in an ideal fluid. Integration of the Clebsch case. A multi-dimensional solid

The equations of motion of a solid in an ideal fluid have the form (see [30])

$$
\left\{\begin{array}{l}
\dot{p}_{1}=p_{2} \frac{\partial H}{\partial l_{3}}-p_{3} \frac{\partial H}{\partial l_{2}} . \\
\dot{p}_{2}=p_{3} \frac{\partial H}{\partial l_{1}}-p_{1} \frac{\partial H}{\partial l_{3}}, \\
\dot{p}_{3}=p_{1} \frac{\partial H}{\partial l_{2}}-p_{2} \frac{\partial H}{\partial l_{1}}, \\
\dot{l}_{1}=p_{2} \frac{\partial H}{\partial p_{3}}-p_{3} \frac{\partial H}{\partial p_{2}}+l_{2} \frac{\partial H}{\partial l_{3}}-l_{3} \frac{\partial H}{\partial l_{2}}, \\
\dot{l}_{2}=p_{3} \frac{\partial H}{\partial p_{1}}-p_{1} \frac{\partial H}{\partial p_{3}}+l_{3} \frac{\partial H}{\partial l_{1}}-l_{1} \frac{\partial H}{\partial l_{3}} \\
\dot{l}_{3}=p_{1} \frac{\partial H}{\partial p_{2}}-p_{2} \frac{\partial H}{\partial p_{1}}+l_{1} \frac{\partial H}{\partial l_{2}}-l_{2} \frac{\partial H}{\partial l_{1}}
\end{array}\right.
$$

where $H$ is the Hamiltonian. These equations have the obvious integrals

(5.4.2) $I_{1}=H, I_{2}=p_{1}^{2}+p_{2}^{2}+p_{3}^{2}, I_{3}=p_{1} l_{1}+p_{2} l_{2}+p_{3} l_{3}$.

$H$ is a quadratic form

$$
2 H=\sum_{j, k}\left(a_{j k} l_{j} l_{k}+2 b_{j k} l_{j} p_{k}+c_{j k} p_{j} p_{k}\right),
$$

then (5.4.1) is the equation of the geodesics of the right-invariant metric on the group $E(3)$ of motions of three-dimensional Euclidean space. The system (5.4.1) is the Hamiltonian on the orbits given by $I_{2}=$ const, $I_{3}=$ const of the coadjoint representation of $E(3)$. To integrate this system is suffices to have one more integral. It can be found trivially in the symmetric case, where (5.4.1) can be integrated in elliptic functions (see [30]). For the motion of a solid of general (asymmetric) form the following cases of integrability are known:

1) The case of Clebsch

$$
2 H=a_{1} l_{1}^{2}+a_{2} l_{2}^{2}+a_{3} l_{3}^{2}+c_{1} p_{1}^{2}+c_{2} p_{2}^{2}+c_{3} p_{3}^{2},
$$

with

$$
\frac{c_{2}-c_{3}}{a_{1}}+\frac{c_{3}-c_{1}}{a_{2}}+\frac{c_{1}-c_{2}}{a_{3}}=0 .
$$

A fourth integral has the form

$$
2 I_{4}=\lambda\left(l_{1}^{2}+l_{2}^{2}+l_{3}^{2}\right)+\left(a_{1} p_{1}^{2}+a_{2} p_{2}^{2}+a_{3} p_{3}^{2}\right),
$$

where the constant $\lambda$ is determined by the conditions

$$
\lambda=\frac{a_{1}\left(a_{2}-a_{3}\right)}{c_{2}-c_{3}}=\frac{a_{2}\left(a_{3}-a_{1}\right)}{c_{3}-c_{1}}=\frac{a_{3}\left(a_{1}-a_{2}\right)}{c_{1}-c_{2}}
$$

(the equations (5.4.5) and (5.4.7) are equivalent). 
2) The case of Lyapunov-Steklov-Kolosov

$$
\begin{aligned}
2 H=a_{1} l_{3}^{2}+a_{2} l_{2}^{2}+a_{3} l_{3}^{2}+c_{1} p_{1}^{2}+c_{2} p_{2}^{2}+ & c_{3} p_{3}^{2}+ \\
& +2 b_{1} p_{1} l_{1}+2 b_{2} p_{2} l_{2}+2 b_{3} p_{3} l_{3},
\end{aligned}
$$

with

$$
\begin{gathered}
b_{j}=\mu\left(a_{1} a_{2} a_{3}\right) a_{j}^{\mathbf{1}}+\gamma(j=1,2,3), \\
c_{\mathbf{1}}=\mu^{2} a_{1}\left(a_{2}-a_{3}\right)^{2}+v^{\prime}, \ldots
\end{gathered}
$$

A fourth integral has the form

$$
\begin{aligned}
2 I_{4}=l_{1}^{2}+l_{2}^{2}+l_{3}^{2}-\mu\left(a_{1} p_{1} l_{1}+a_{2} p_{2} l_{2}\right. & \left.+a_{3} p_{3} l_{3}\right)+ \\
& +C_{1} p_{1}^{2}+C_{2} p_{2}^{2}+C_{3} p_{3}^{2}
\end{aligned}
$$

where

$$
C_{1}=\mu^{2}\left(a_{2}-a_{3}\right)^{2}, \ldots
$$

These cases exhaust all the possibilities when the system (5.4.1) with a Hamiltonian of the type (5.4.3) has a fourth integral that is quadratic in $l$ and $p$. The equations (5.4.1) for the Clebsch case are integrated in terms of $\theta$-functions in [32]-[34]; the author has recently become aware that a complete integration of the Lyapunov-Steklov case was given as early as 1900 by Kötter in [52].

We introduce coordinates of the level surface of the integrals $I_{1}, \ldots, I_{4}$ for the Clebsch case. Taking a linear combination of the integrals $I_{1}$ and $I_{2}$ and changing $a_{i} \mapsto \lambda a_{i}(i=1,2,3)$, we rewrite the equations of this surface in the form

$$
\left\{\begin{array}{l}
p_{1}^{2}+p_{2}^{2}+p_{3}^{2}=k_{0}, \\
a_{1} p_{1}^{2}+a_{2} p_{2}^{2}+a_{3} p_{3}^{2}+l_{1}^{2}+l_{2}^{2}+l_{3}^{2}=k_{1}, \\
-\left(a_{2} a_{3} p_{1}^{2}+a_{1} a_{3} p_{2}^{2}+a_{1} a_{2} p_{3}^{2}\right)+a_{1} l_{1}^{2}+a_{2} l_{2}^{2}+a_{3} l_{3}^{2}=k_{2}, \\
p_{1} l_{1}+p_{2} l_{2}+p_{3} l_{3}=k_{3},
\end{array}\right.
$$

where $k_{0}, \ldots, k_{3}$ are constants. Let $s_{1}, \ldots, s_{4}$ be the roots of the equation

$$
\begin{aligned}
k_{0}^{2}\left[s^{2}-s\left(a_{1}+a_{2}+a_{3}\right)\right]+k_{1} s & -k_{2}+ \\
& +2 k_{3} \sqrt{\left(s-a_{1}\right)\left(s-a_{2}\right)\left(s-a_{3}\right)}=0 .
\end{aligned}
$$

We set

$$
\psi(s)=\left(s-s_{1}\right) \ldots\left(s-s_{4}\right)
$$

and pass from $p_{j}$ and $l_{k}$ to the variables $\xi_{j}^{+}$and $\xi_{j}^{-}$, setting

$$
\begin{aligned}
(5.4 .14) \xi_{k}^{ \pm}=p_{k}\left[\frac{\sqrt{\left(s_{1}-a_{1}\right)\left(s_{1}-a_{2}\right)\left(s_{1}-a_{3}\right)}}{\sqrt{s_{1}-a_{k}} \sqrt{\psi^{\prime}\left(s_{1}\right)}}\right. & \left. \pm i \frac{\sqrt{\left(s_{2}-a_{1}\right)\left(s_{2}-a_{2}\right)\left(s_{2}-a_{3}\right)}}{\sqrt{s_{2}-a_{k}} \sqrt{\psi^{\prime}\left(s_{2}\right)}}\right]+ \\
& +l_{k}\left[\frac{\sqrt{s_{1}-a_{k}}}{\sqrt{\psi^{\prime}\left(s_{1}\right)}} \pm i \frac{\sqrt{s_{2}-a_{k}}}{\sqrt{\psi^{\prime}\left(s_{2}\right)}}\right] .
\end{aligned}
$$


Let $z_{1}$ and $z_{2}$ be the roots of the equation

$$
\frac{\left(\xi_{\bar{l}}\right)^{2}}{d_{1}^{2}-z}+\frac{\left(\xi_{\overline{2}}\right)^{2}}{d_{2}^{2}-z}+\frac{\left(\xi_{\overline{3}}\right)^{2}}{d_{3}^{2}-z}=0
$$

("elliptic coordinate"), where

$$
d_{h}=\frac{\frac{\sqrt{s_{3}-a_{k}}}{\sqrt{\psi^{\prime}\left(s_{3}\right)}}+i \frac{\sqrt{s_{4}-a_{h}}}{\sqrt{\psi^{\prime}\left(s_{4}\right)}}}{\frac{\sqrt{s_{1}-a_{k}}}{\sqrt{\psi^{\prime}\left(s_{1}\right)}}+i \frac{\sqrt{s_{2}-a_{k}}}{\psi^{\prime}\left(s_{2}\right)}} .
$$

It is not hard to express the coordinates $\xi_{k}^{ \pm}$(hence also $p_{j}$ and $l_{k}$ ) in terms of $z_{1}$ and $z_{2}$. This means that $z_{1}$ and $z_{2}$ are the required coordinates on (5.4.11). A direct calculation shows that the equations (5.4.1) in the Clebsch case can be rewritten on $(5.4 .11)$ in the form

$$
\text { (5.4.17) } \quad \frac{d z_{1}}{d t}=\frac{\left(a z_{2}+b\right) \sqrt{R\left(z_{1}\right)}}{z_{2}-z_{1}}, \quad \frac{d z_{2}}{d t}=\frac{\left(a z_{2}+b\right) \sqrt{R\left(z_{2}\right)}}{z_{1}-z_{2}},
$$

where $R(z)$ is a polynomial of degree 5 , and

$$
R(z)=z\left(z-d_{1}^{2}\right)\left(z-d_{2}^{2}\right)\left(z-d_{3}^{2}\right)\left(z-d_{1}^{2} d_{2}^{2} d_{3}^{2}\right) ;
$$

we do not give the explicit form of the constants $a$ and $b$. The system $(5.4 .17)$ is a linear combination of $(2.4 .55)$ and $(2.4 .17)$ and can be integrated by means of the Abel transformation $\Gamma \stackrel{A}{\rightarrow} J(\Gamma)$ where the Riemann surface $\Gamma$ of genus 2 has the form

$$
w^{2}=R(z) \text {. }
$$

Consequently, the equations (5.4.1) in the Clebsch case are integrated in $\theta$-functions of genus 2 .

We now give one more example of completely integrable systems: the Euler equations of motion of a multidimensional solid, which have the form (see [44])

$$
\dot{M}=[\Omega, M],
$$

where

$$
M=I \Omega+\Omega I,
$$

and $I$ is the inertia operator of the solid, and

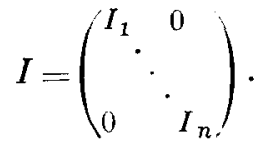

The complete integrability of (5.4.20) for all $n$ was proved by Manakov in [41]. The proof is based on a representation of this system in the equivalent form

$$
[A, \dot{V}]=[[A, V],[B, V]]
$$


where
$\left(5.4 .23^{\prime}\right)$
$[B, V]=\Omega, \quad A=I^{2}, \quad B=I$.

The systems (5.4.23) have been explicitly integrated by the present author in [42]. They all have a commutative representation of the form

$$
\left[\frac{d}{d t}-[B, V]+z B, \quad z A-[A, V]\right]=0
$$

on matrices depending on a superfluous parameter $z$; consequently, their solutions can be expressed in terms of $\theta$-functions of Riemann surfaces $\Gamma$ of the form

$$
\operatorname{det}(z A-[A, V]-w \cdot 1)=0 \text {. }
$$

The set of these surfaces $\Gamma$ is the same as that of all plane non-singular algebraic curves (in $\mathbf{C} P^{2}$ ) of degree $n$ (their genus is $\left.(n-1)(n-2) / 2\right)$ and their degeneracies. Explicit formulae for a general solution of (5.4.23) can be obtained from [42] and have the form $V=\left(V_{i j}\right)$, where

$$
\begin{gathered}
v_{i j}= \pm \frac{\lambda_{i}}{\lambda_{j}} \frac{\theta\left(A\left(P_{i}\right)-A\left(P_{j}\right)+t U+z_{0}\right)}{\theta\left(t U+z_{0}\right) \varepsilon\left(P_{i}, P_{j}\right)} \quad(i \neq j) \\
{\left[\varepsilon(P, Q]^{-1}=\frac{\sqrt{\partial_{U(P)} \theta[v](0) \partial_{U(Q)} \theta[v](0)}}{\theta[\nu](A(\bar{P})-A(Q))},\right.} \\
\lambda_{i}=\lambda_{i}^{0} \exp \left\{t \sum_{k \neq i} c_{i}^{k} b_{k}\right\}, \\
c_{i}^{k}=-\left.\frac{d}{d P} \log \varepsilon\left(P, P_{i}\right)\right|_{P=P_{k}} .
\end{gathered}
$$

Here $\lambda_{l}^{0}, \ldots, \lambda_{n}^{0}$ are arbitrary non-zero constants; the $\theta$-function is constructed from a curve of the form $(5.4 .25) ; P_{1}, \ldots, P_{n}$ are the points at infinity on this curve, where $w / z \rightarrow a_{i}$ as $P \rightarrow P_{i}$; the vector $U$ has the form

$$
U=\sum_{j=1}^{n} b_{j} U\left(P_{j}\right)
$$

where $U(P)$ is a period vector of differentials $\Omega_{P}$ with a double pole at $P ; z$ is an arbitrary vector; and finally, $v$ is any non-degenerate odd half-period (that is, $\operatorname{grad} \theta[v](0) \neq 0$ ).

\section{APPENDIX}

\section{THE PERIODIC NON-ABELIAN TODA CHAIN AND ITS TWO-DIMENSIONAL GENERALIZATION}

\section{I.M. Krichever}

The equations of a non-Abelian Toda chain were suggested by Polyakov, who found polynomial integrals for them. These equations, which have the form

$$
\partial_{t}\left(\partial_{t} g_{n} \cdot g_{n}^{-1}\right)=g_{n-1} g_{n}^{-1}-g_{n} g_{n+1}^{-1}, \quad \partial_{t}=\frac{\partial}{\partial t},
$$


where the $g_{n}$ are matrices of order $l$, admit a commutative representation of Lax type $\partial_{t} L=[P, L]$. Here

$$
\begin{gathered}
L \psi_{n}=g_{n} g_{n+1}^{-1} \psi_{n+1}-\dot{g}_{n} g_{n}^{-1} \psi_{n}+\psi_{n-1}, \quad \dot{g}_{n}=\partial_{t} g_{n}, \\
P \psi_{n}=\frac{1}{2}\left(g_{n} g_{n+1}^{-1} \psi_{n+1}+\dot{g}_{n} g_{n}^{-1} \psi_{n}-\psi_{n-1}\right) .
\end{gathered}
$$

Using this representation, explicit expressions in terms of Riemann $\theta$-functions have been obtained in the present survey for periodic solutions, $g_{n+N}=g_{n}$, of the equations (1).

In contrast to the continuous case when the algebraic-geometric constructions give only the so-called finite-zone solutions, in a difference version all the periodic solutions of the Lax equations turn out to be algebraic-geometric. This is connected with the fact that shift by a period, which commutes with $l$, is a difference operator.

In [46] the present author obtained a classification of commuting difference operators (see also [47]). In the same paper a construction of quasiperiodic solutions of difference operators of Zakharov-Shabat type and Lax type was proposed. Apart from general solutions of similar type, the non-abelian Toda chain has separatrix families of solutions or, in the terminology of $[14]$, finite-zone solutions of rank $l>1$. Their dimension is more than half the dimension of the phase space.

First we recall the scheme of integration $([15],[46])$ of the "ordinary" Toda chain

$$
\left\{\begin{array}{l}
\dot{v}_{n}=c_{n+1}-c_{n}, \\
\dot{c}_{n}=c_{n}\left(v_{n}-v_{n-1}\right) .
\end{array}\right.
$$

Let $R$ be a hyperelliptic Riemann surface of genus $g$ of the form

$$
w^{2}=\prod_{i=1}^{2 g+2}\left(z-z_{i}\right)
$$

$P^{+}$and $P^{-}$the points of $R$ of the form $P^{ \pm}=(\infty, \pm)$. To integrate the system (4) we introduce the Baker-Akhiezer function $\psi(n, t, P)$ which is, meromorphic on $R$ everywhere except for at $P^{+}$and $P^{-}$, where it has $g$ poles and as $P \rightarrow P^{ \pm}$, an asymptotic expansion of the form

$$
\text { (6) }\left.\quad \psi_{i}^{\prime}(n, t, P)\right|_{P \rightarrow P^{ \pm}}=i^{n} \lambda_{n}^{ \pm 1} z^{ \pm n}\left(1+\xi_{1}^{ \pm}(n, t) z^{-1}+\ldots\right) \exp \left(\mp \frac{t z}{2}\right) .
$$

For this function there are difference operators $L=\left(L^{n m}\right)$ and $A=\left(A^{n m}\right)$ such that

$$
\frac{\partial \psi}{\partial t}=A \psi, \quad L \psi=z \psi
$$


These operators have the form

$$
\begin{aligned}
& L^{n m}=-i \sqrt{c_{n+1}} \delta_{n, m-1}+v_{n} \delta_{n, m}+i \sqrt{c_{n}} \delta_{n, m+1}, \\
& A^{n m}=\frac{i}{2} \sqrt{c_{n+1}} \delta_{n, m-1}+w_{n} \delta_{n, m}+\frac{i}{2} \sqrt{c_{n}} \delta_{n, m+1} .
\end{aligned}
$$

Here $w_{n}-w_{n-1}=\frac{1}{2}\left(v_{n}-v_{n-1}\right)-\frac{1}{2}\left(\log c_{n}\right)$, and

$$
\begin{gathered}
\sqrt{c_{n}}=\lambda_{n-1} / \lambda_{n}, \\
v_{n}=\xi_{1}^{+}(n+1, t)-\xi_{1}^{+}(n, t) .
\end{gathered}
$$

The compatibility condition for (7) coincides with the equations of the Toda chain. Expressing the Baker-Akhiezer function (6) in terms of $\theta$-functions of $R$ and calculating the coefficients $\lambda_{n}$ and $\xi_{1}^{+}(n, t)$, we obtain an explicit form of the solutions of the Toda chain:

$$
\begin{gathered}
v_{n}=\frac{d}{d t} \log \frac{\theta\left((n+1) U+t V+z_{0}\right)}{\theta\left(n V+t V+z_{0}\right)}, \\
c_{n}=\frac{\theta\left((n+1) U+V t+z_{0}\right) \theta\left((n-1) U+V t+z_{0}\right)}{\theta^{2}\left(n U+V t+z_{0}\right)} .
\end{gathered}
$$

Here $z_{0}$ is an arbitrary vector; the vectors $U=\left(U_{j}\right)$ and $V=\left(V_{j}\right)$ are determined as follows:

$$
U_{j}=\int_{P^{-}}^{P^{+}} \omega_{j}
$$

$\left(\omega_{1}, \ldots, \omega_{g}\right.$ is a canonical basis of holomorphic differentials on $\left.R\right)$,

$$
2 V_{j}=\oint_{b_{j}} \Omega_{P^{+}}+\oint_{b_{j}} \Omega_{P^{-}}
$$

where $\Omega_{P^{+}}$and $\Omega_{P^{-}}$are normalized differentials of the second kind with a double pole at $P^{+}$and $P^{-}$, respectively.

Periodic solutions of the Toda chain with period $N$ are distinguished in our system as follows: $R$ must have the form

$$
w^{2}=\left(P_{N}(z)+1\right)\left(P_{N}(z)-1\right)
$$

where $P_{N}(z)$ is a polynomial. We emphasize that all periodic solutions of the Toda chain are obtained in this way.

1. Thus, we consider periodic solutions of (1). The restriction of $L$ to the space of eigenfunctions of the shift operator by a period, that is, $\psi_{n+N}=w \psi_{n}$, where $\psi_{n}$ is an $l$-dimensional vector, is a finite-dimensional linear operator. Its matrix has the form

$$
\tilde{L}=\left(\begin{array}{lllllll}
b_{N-1} & 1 & 0 & \ldots & & 0 & w a_{N-1} \\
a_{N-2} & b_{N-2} & 1 & \ldots & & 0 & 0 \\
\cdots & \ldots & \ldots & \ldots & \ldots & \ldots & \ldots \\
0 & 0 & & \ldots & a_{1} & b_{1} & 1 \\
w^{-1} & 0 & & \ldots & 0 & a_{0} & b_{0}
\end{array}\right),
$$

where the block $(l \times l)$-elements are $b_{n}=-\dot{g}_{n} g_{n}^{-1}, a_{n}=g_{n} g_{n-1}^{-1}$. 
It follows from the Lax representation that the coefficients of the polynomial $Q(w, \lambda)=\operatorname{det}(\widetilde{L}-\lambda \cdot 1)$ are the integrals of (1). However, in contrast to the Abel case they are not independent.

Lemma 1 . The polynomial $Q(w, \lambda)$ has the form

$$
\begin{array}{r}
\left(w-\lambda^{N}\right)^{l}+\left(w^{-1}-\lambda^{N}\right)^{l}+\sum_{k=1}^{l-1}\left(r_{k}^{+}(\lambda)\left(w-\lambda^{N}\right)^{k}+r_{k}^{-}(\lambda)\left(w^{-1}-\lambda^{N}\right)^{k}\right)- \\
-R_{0}(\lambda)+\sum a_{i j} \lambda^{i} w^{j}
\end{array}
$$

The last summation is over the pairs $i, j$ such that. $i \geqslant 0, i+N|j| \leqslant(N-1) l$. The polynomials $r_{k}^{ \pm}$have only $k$ non-zero coefficients:

$$
r_{k}^{ \pm}(\lambda)=\sum_{i=(N-1)(l-k)-k+1}^{(N-1)(l-k)} b_{k i}^{+} \lambda^{i}
$$

The coefficients $a_{i j}$ and $b_{k i}^{ \pm}$are a complete system of integrals in involution with the single relation

$$
R_{0}(\lambda)+\left(-\lambda^{N}\right)^{l}=\sum_{k} r_{k}^{+}(\lambda)\left(-\lambda^{N}\right)^{k}=\sum_{k} r_{k}^{-}(\lambda)\left(-\lambda^{N}\right)^{k}
$$

The number of independent integrals is $N l^{2}-l+1$.

The restrictions on the form $Q(w, \lambda)$ are equivalent to the following condition: all the roots $w$ of $Q(w, \lambda)=0$ for large $\lambda$ must be expandable in Laurent series in $\lambda^{-1}$, one half of them must be of the form $\lambda^{N}+O\left(\lambda^{N-1}\right)$, and the other half of the form $\lambda^{-N}+O\left(\lambda^{-N-1}\right)$.

We consider the algebraic curve $R$, given in $\mathrm{C}^{2}$ by the equation $Q(w, \lambda)=0$. In general position we may assume that it is non-singular and that $Q(w, \lambda)=0$ for almost all $\lambda$ has $2 l$ distinct roots $w_{j}$. Then to each point $P$ of $R$, that is, $P=\left(w_{j}, \lambda\right)$ there corresponds the unique eigenvector $\varphi_{n}(t)=\left(\varphi_{n}^{1}, \ldots, \varphi_{n}^{l}\right)^{t}$, normalized by the condition $\varphi_{0} \equiv 1$. All remaining coordinates $\varphi_{n}(t)$ are meromorphic functions on $\mathfrak{R}$. Their poles lie at the points $\gamma_{i}(t)$, where the left upper principal minor $\widetilde{L}-\lambda \cdot 1$ vanishes and $[\operatorname{rank}(\widetilde{L}-\lambda \cdot 1)=N l-1]$.

Lemma 2. The number of poles $\gamma_{i}(t)$ is $N l^{2}-l^{2}=g+l-1$, where $g$ is the genus of $R$.

Thus, to every set of initial conditions $g_{n}(0)$ and $\dot{g}_{n} g_{n}^{-1}(0)$ there corresponds a curve $R$, that is, a polynomial $Q$ and a set of $N l^{2}-l^{2}$ points $\gamma_{i}(0)$ on it. The solutions differing by a transformation $g_{n} \rightarrow G g_{n}$, where $G$ is a constant matrix, are the kernel of this mapping.

We consider the problem of recovering $L$ from the indicated data.

Let $Q$ be as in Lemma 1 . Then $R$ is compactified at infinity in $\lambda$ by the points $P_{j}^{ \pm}$at which $w$ has poles of order $N$ and zeros of multiplicity $N$, respectively. 
Lemma 3. For any set of $N l^{2}-l$ points $\gamma_{i}$ in general position there exists one and only one vector-function $\psi_{n}(t, P)$ with the following properties:

$1^{\circ}$ it is meromorphic on $R$ except at $P_{j}^{ \pm}$with poles at $\gamma_{i}$;

$2^{\circ}$ if we form from $\psi_{n}\left(t, \lambda_{j}^{ \pm}\right)$as columns, the matrices $\psi_{n}^{ \pm}(t, \lambda)$ then they have the form

$$
\psi_{n}^{ \pm}(t, \lambda)=\lambda^{ \pm n}\left(\sum \xi_{n, s}^{\mp}(t) \lambda^{-s}\right) e^{\mp \lambda t / 2}, \quad \xi_{n, 0}^{-}=1
$$

Here the $\lambda_{j}^{ \pm}$are inverse images of $\lambda$ in a neighbourhood of $P_{j}^{ \pm}$.

Lemma 4. The function $\psi_{n}(t)$ satisfies the equations

where $g_{n}=\xi_{n, 0}^{+}$.

$$
L \psi_{n}=\lambda \psi_{n}, \quad\left(\partial_{t}-P\right) \psi_{n}=0,
$$

The functions $\varphi_{n}(t)$ and $\psi_{n}(t)$ differ in the normalization $\rho_{n}(t)=\psi_{n}\left(\psi_{0}^{l}\right)^{-1}$.

Corollary. The matrices $g_{n}$ satisfy the equation (1). By the restrictions to $Q$, the thus constructed solutions are periodic, $g_{n+N}=g_{n}$.

For $\psi_{n}$ we can construct formulae of Baker-Its type, by analogy with [15]. Calculating $\xi_{n, 0}^{ \pm}$from them we obtain the following result.

Theorem 1. For any polynomial of the form (16) and any set of $\mathrm{Nl}^{2}-\mathrm{l}^{2}$ points $\gamma_{i}$ in general position the functions

$$
g_{n}(t)=\left(g_{n}^{-}\right)^{-1} g_{n}^{+} c^{n}
$$

are periodic solutions (1), where the matrix elements of $g_{n}^{ \pm}$are

$$
g_{n, i j}^{ \pm}=\theta\left(\omega_{j}^{ \pm}+\vec{U} n+\vec{V} t+\vec{Z}_{i}\right) \theta^{-1}\left(\omega_{j}^{ \pm}+\vec{Z}_{i}\right) \text {. }
$$

The constant vectors $\vec{U}$ and $\vec{V}$ are given by the periods of differentials of the third and second kinds with poles at $P_{j}^{ \pm} ; \omega_{j}^{ \pm}$are the images of the points $P_{j}^{ \pm}$under the Abel transformation, and the $\vec{Z}_{i}$ are the images of the divisors $\gamma_{1}, \ldots, \gamma_{g-1}, \gamma_{g+i}, 1 \leqslant i \leqslant l$. also under the Abel transformation. The constant $c$ is determined from the periodicity condition $g_{N}=g_{0}$.

The general solution has the form $G_{1} g_{n} G_{2}$, where the $G_{i}$ are fixed matrices.

Remark. The calculation of all of the parameters in the formulae of the theorem from the initial data $g_{n}(0)$ and $g_{n} g_{n}^{-1}(0)$ only uses quadratures and a solution of algebraic equations, and the latter is necessary only to find the $\vec{Z}_{i}$. All the remaining parameters $\vec{\omega}_{j}^{ \pm}, U, \vec{V}$, etc. can be expressed by quadratures in terms of the integrals.

2. Considering special cases of multiple eigenvalues of $L$ and a shift by a period, we restrict ourselves to the case of maximal degeneracy of multiplicity $l$. Then the polynomial $Q$ has the form $Q(w, \lambda)=Q_{1}^{l}(w, \lambda)$, $Q_{1}=w+w^{-1}+\sum_{i=0}^{N} a_{i} \lambda^{i}$. To each point of the hyperelliptic curve $R$ given 
by $Q_{1}(w, \lambda)=0$ there corresponds an $l$-dimensional subspace of joint eigenfunctions. Let $\psi_{n}(t, P)$ be the matrix whose columns form a basis in this subspace, normalized by the condition $\psi_{0}(0, P)=1$. Then $\psi_{n}$ is a meromorphic matrix, having $l N$ poles $\gamma_{s}$, and

$$
\varphi_{n, s}^{i j}=\alpha_{s}^{j} \cdot \varphi_{n, s}^{i l} ; \quad \varphi_{n, s}^{i j}=\operatorname{res} \gamma_{s} \psi_{n}^{i j},
$$

where the $\alpha_{s}^{i}$ are constants independent of $n$ and $t$. In a neighbourhood $P^{ \pm}$ of the inverse images of $\lambda=\infty, \psi_{n}$ has the form

$$
\Psi_{n}^{ \pm}(t, \lambda)=\lambda^{ \pm n}\left(\sum_{s=0}^{\infty} \xi_{n, s}^{ \pm}(t) \lambda^{-s}\right) e^{\mp \lambda t / 2} .
$$

Lemma 5. For any set of data $\left(\gamma_{s}, \alpha_{s}^{j}\right)$ (which are called, as in [14], the Tyurin parameters) in general position there exists one and only one matrix function $\psi_{n}$ satisfying (20) and (21) and normalized by the requirement $\xi_{n, 0}^{-} \equiv 1$.

Just as above, $\xi_{n, 0}$ can be proved to be a periodic solution of (1).

3 . In conclusion we give a construction of the periodic solutions of the equations

$$
\left(\partial_{t}^{2}-\partial_{x}^{2}\right) \varphi_{n}=e^{\varphi_{n}-\varphi_{n-1}}-e^{\varphi_{n+1}-\varphi_{n}},
$$

to which, as was found in [48], the two-dimensional version by ZakharovShabat of the Lax pair for the Abelian Toda chain reduces. These equations generalize, besides the equations of the chain itself, the sine-Gordon equation corresponding to the periodic solutions $\varphi_{n+2}=\varphi_{n}$.

We consider a non-singular algebraic curve $R$ of genus $g$ with two distinguished points $P^{ \pm}$.

Lemma 6. For any set of points $\gamma_{1}, \ldots, \gamma_{g}$ in general position there exist unique functions $\psi_{n}\left(z_{+}, z_{-}, P\right)$ such that:

$1^{\circ}$ they are meromorphic except at $P^{ \pm}$with poles at $\gamma_{1}, \ldots, \gamma_{g}$;

$2^{\circ}$ in a neighbourhood of $P^{ \pm}$they are representable in the form

$$
\psi_{n}\left(z_{+}, z_{-}, P^{ \pm}\right)=e^{h z} \pm\left(\sum_{s=0}^{\infty} \xi_{n, s}^{ \pm}\left(z_{+}, z_{-}\right) k^{-s}\right) k^{ \pm n} ;
$$

where $\xi_{n, 0}^{ \pm}=1$ and $k^{-1}=k^{-1}\left(P^{ \pm}\right)$are local parameters in neighbourhoods of $P^{ \pm}$.

Lemma 7. The following equalities hold:

$$
\partial_{z_{+}} \psi_{n}=\psi_{n+1}+\left(\partial_{z_{+}} \varphi_{n}\right) \psi_{n}, \quad \partial_{z_{-}} \psi_{n}=e^{\varphi_{n}-\varphi_{n-1}} \psi_{n-1} ; \quad e^{\varphi_{n}}=\xi_{n, 0}^{-} .
$$

The compatibility conditions of these equalities are equivalent to the equations

$$
\frac{\partial^{2}}{\partial z_{+} \partial z_{-}} \varphi_{n}=e^{\varphi_{n}-\varphi_{n-1}}-e^{\varphi_{n+1}-\varphi_{n}},
$$

which coincide with (22) written in conical variables. 
Theorem 2. For each non-singular complex curve $\mathbb{R}$ with two distinguished points the formula

$$
\varphi_{n}=\log \frac{\theta\left(\omega^{+}+U_{1} t+U_{2} x+U_{3} n+W\right)}{\theta\left(\omega^{-}+U_{1} t+U_{2} x+U_{3} n+W\right)}+\log \frac{\theta\left(\omega^{-}+W\right)}{\theta\left(\omega^{+}+W\right)}+n c
$$

gives a solution of the equations (22).

Here $\omega^{ \pm}=\left(\omega_{1}^{ \pm}, \ldots, \omega_{g}^{ \pm}\right)$are the images of $P^{ \pm}$under the Abel transformation; the vectors $U_{i}$ depend on the points $P^{ \pm}$and are the period vectors of Abelian differentials of the second and third kinds with appropriately chosen singularities at $P^{ \pm}$(see, by analogy, [15]).

Let us distinguish the periodic solutions $\varphi_{n+N}=\varphi_{n}$ among the solutions thus constructed. For this purpose there must be a function $E(P)$ on $R$ having a pole of order $N$ and a zero of order $N$ at $P^{ \pm}$.

Suppose $R$ is given in $\mathbf{C}^{2}$ by the equation

$$
w^{N}-E^{m}+E\left(\sum a_{i j} E^{i} w^{j}\right)=0
$$

$N(i+1)+m j \leqslant N m-2 ; N$ is prime to $m$. This is an $N$-sheeted cover of the $E$-plane, and over $E=0$ and $E=\infty$ all the sheets are glued, that is, the function $E(P)$ given by the projection of $R$ has the required properties.

Corollary. Suppose that $\mathbb{R}$ is of the form (24); then the formulae (23) give periodic solutions of (22).

Remark (Dubrovin). The methods developed in Chapter 4 of the present survey allow us, in particular, to make the formula (23) for the solutions of (22) effective. By substituting (23) in (22) we obtain after simple transformations the following relation:

$$
a \frac{\theta\left(U_{3}+W\right) \theta\left(U_{3}-W\right)}{\theta^{2}(W)}=b+\partial_{U\left(P^{*}\right)} \partial_{U\left(P^{-}\right)} \log \theta(W) .
$$

Here $W$ is an arbitrary $g$-dimensional vector; $U(P)$ for each $P \in \mathbb{R}$ is a period vector of a differential with a double pole at $P\left(2 U_{1,2}=U\left(P^{+}\right) \pm U\left(P^{-}\right)\right)$; the constants $a$ and $b$ have the form

$$
a=\varepsilon^{-2}\left(P^{+}, P^{-}\right), \quad b=\left.\frac{d}{d P} \frac{d}{d Q} \log \varepsilon(P, Q)\right|_{P=P^{+} Q=P^{-}}
$$

( $\varepsilon(P, Q)$ is defined by (5.4.27)). This is a standard identity in the theory of Abelian functions (see [8], (39)). Applying the addition theorem to (25), we obtain the following system (in the notation of Chapter IV):

$$
a \hat{\theta}[n]\left(2 U_{3}\right)=b \hat{\theta}[n](0)+\partial_{U\left(P^{+}\right)} \partial_{U\left(P^{-}\right)} \hat{\theta}[n](0),
$$

where

$$
n \in \frac{1}{2}\left(Z_{2}\right)^{g}
$$


Here $U_{3}=A\left(P^{+}\right)-A\left(P^{-}\right)$, therefore, the system (26), together with (4.2.4), allows us to recover from the period matrix not only the canonical equations of the curve $R$, but also the image of the Abel transformation $A: R \rightarrow J(R)$ (although, for this we have to solve the transcendental equation (26) for $U_{3}$ )).

\section{References}

[1] G. Springer, Introduction to Riemann surfaces, Addison-Wesley, Reading, MA, 1957. MR 19-1169

Translation: Vvedenie v teoriyu rimanovykh poverkhnostei, Izdat. Inostr. Lit., Moscow 1960. MR 23 \# A318.

[2] N.G. Chebotarev, Teoriya algebraicheskikh funktsii (Theory of algebraic functions), OGIZ, Moscow 1948.

[3] I.R. Shafarevich, Osnovy algebraicheskoi geometrii, Nauka, Moscow 1972. MR 51 \# 3162.

Translation: Basic algebraic geometry, Springer-Verlag, Berlin-Heidelberg-New York 1974. MR 51 \# 3163.

[4] P.A. Griffiths and J. Harris, Principles of algebraic geometry, Wiley-Interscience, New York 1978. MR 80b:14001.

[5] J. Igusa, Theta-functions, Springer-Verlag, Berlin-Heidelberg-New York 1972. MR 48 \# 3972.

[6] A. Krazer, Lehrbuch der Thetafunktionen, reprint, Chelsea, New York 1970.

[7] H.F. Baker, Abelian functions, University Press, Cambridge 1897.

[8] J.D. Fay, Theta-functions on Riemann surfaces, Lecture Notes in Math. 352 (1973). MR 49 \# 569.

[9] E.I. Zverovich, Boundary-value problems of the theory of analytic functions, Uspekhi Mat. Nauk 26:1 (1971), 113-181.

$=$ Russian Math. Surveys 26:1 (1971), 117-192.

[10] V.V. Golubev, Lektsii po integrirovaniyu uravnenii dvizheniya tyazhelogo tverdogo tela okolo nepodvizhnoi tochki (Lectures on the integration of equations of motion of a heavy solid about a fixed point), Gostekhizdat, Moscow 1953. MR 15-904.

[11] A.I. Markushevich, Vvedenie v klassicheskuyu teoriyu abelevykh funktsii (Introduction to the classical theory of Abelian functions), Nauka, Moscow 1979. MR $81 \mathrm{c}: 14024$.

[12] H. Bateman and A. Erdélyi, Higher transcendental functions, Vol. 3, McGraw-Hill 1955. MR 16-586.

Translation: Vysshie transtsendentnye funktsii, Tom 3, Nauka, Moscow 1967.

[13] S.P. Novikov, (Editor) Teoriya solitonov (Theory of solitons), Nauka, Moscow 1980,

[14] I.M. Krichever and S.P. Novikov, Holomorphic bundles over algebraic curves and nonlinear equations, Uspekhi Mat. Nauk 35:6 (1980), 47-68.

$=$ Russian Math. Surveys 35:6 (1980), 53-79.

[15] - Methods of algebraic geometry in the theory of non-linear equations, Uspekhi Mat. Nauk 32:6 (1977), 183-208. MR 58 \# 24353.

= Russian Math. Surveys 32:6 (1977), 185-213.

[16] - Integration of non-linear equations by methods of algebraic geometry, Funktsional. Anal. i Prilozhen. 11:1 (1977), 15-32. MR 58 \# 13168. = Functional Anal. Appl. 11:1 (1977), 12-26.

[17] B.A. Dubrovin, V.B. Matveev and S.P. Novikov, Non-linear equations of Kortewegde Vries type, finite-zone operators, and Abelian varieties, Uspekhi Mat. Nauk 31:1 (1976), 55-136. MR 55 \# 899.

= Russian Math. Surveys 31:1 (1976), 59-146. 
[18] N.I. Akhiezer, A continuous analogy of orthogonal polynomials on a system of integrals, Dokl. Akad. Nauk SSSR 141 (1961), 263-266.

= Soviet Math. Dokl. 2 (1961), 1409-1412.

[19] H.F. Baker, Note on the foregoing paper "Commutative ordinary differential operators" by J.L. Burchnall and T.W. Chaundy, Proc. Royal Soc. London A118 (1928), 584-593.

[20] S.P. Novikov, A periodic problem for the Korteweg-de Vries equation, I, Funktsional. Anal. i Prilozhen. 8:3 (1974), 54-66.

$=$ Functional Anal. Appl. 8 (1974), 236-246.

[21] B.A. Dubrovin, I.M. Krichever and S.P. Novikov, The Schrödinger equation in a magnetic field and Riemann surfaces, Dokl. Akad. Nauk SSSR 229 (1976), 15-18.

= Soviet Math. Dokl. 17 (1976), 947-951.

[22] I.M. Krichever, Elliptic solutions of the Kadomtsev-Petviashvili equation and integrable systems of particles on a straight line, Funktsional. Anal. i Prilozhen. 14:4 (1980), 45-54.

= Functional Anal. Appl. 14 (1980), 282-290.

[23] B.A. Dubrovin, On a conjecture of Novikov in the theory of theta-functions and non-linear equations of Korteweg-de Vries and Kadomtsev-Petviashvili type, Dokl. Akad. Nauk SSSR 251 (1980), $541-544$.

= Soviet Math. Dokl. 21 (1980), 469-472.

[24] R. Hirota, Recent developments of direct methods in soliton theory, Preprint, Hiroshima University 1979.

[25] A.N. Tyurin, The geometry of the Poincaré divisor of a Prym variety, Izv. Akad. Nauk SSSR Ser. Mat. 39 (1975), 1003-1043. MR 51 \# 2664.

$=$ Math. USSR-Izv. 9 (1975), $951-986$.

[26] F. Schottky, Úber die Moduln der Thetafunktionen, Acta Math. 27 (1903), $235-288$.

[27] C. Neumann, De problemate quodam mechanico, quod ad primam integralium ultraellipticorum classem revocatur, J. Reine Angew. Math. 56 (1859), 46-63.

[28] J. Moser, Various aspects of integrable Hamiltonian systems, Preprint Courant Institute, New York 1978.

[29] A.P. Veselov, Finite-zone potentials and integrable systems on a sphere with a quadratic potential, Funktsional. Anal. i Prilozhen. 14:1 (1980), 48 -50.

= Functional Anal. Appl. 14 (1980), 37-39.

[30] G.R. Kirchhoff, Mechanik, Berlin 1876.

Translation: Mekhanika, Izdat. Akad. Nauk SSSR, Moscow 1962.

[31] A.M. Perelomov, Some remarks on the integration of equation of motion of a solid in an ideal liquid, Funktsional. Anal. i Prilozhen. 15:2 (1981), 83-85.

= Functional Anal. Appl. 15 (1981), 144-146.

[32] H. Weber, Anwendung det Thetafunktionen zweier Veränderlicher auf die Theorie der Bewegung eines festen Körpers in einer Flüssigkeit, Math. Ann. 14 (1978), 173-206.

[33] F. Kötter, Über die Bewegung eines festen Körpers in einer Flüssigkeit. I, II, J. Reine Angew. Math. 109 (1892), 51-81, 89-111.

[34] V.A. Steklov, $O$ dvizhanii tverdogo tela $v$ zhidkosti (On the motion of a solid in a liquid), Kharkov 1893.

[35] B.A. Dubrovin and S.P. Novikov, A periodic problem for the Korteweg-de Vries and Sturm-Liouville equations. Their connection with algebraic geometry, Dokl. Akad.

Nauk SSSR 219 (1974), 19-22. MR 58 \# 1761.

$=$ Soviet Math. Dokl. 15 (1974), 1597-1601.

[36] S.Yu. Dobrokhotov and V.P. Maslov, Finite-zone almost periodic solutions in VKBapproximations, Sovremennye problemy matematiki 15 (1980), 3-94. 
[37] B.A. Dubrovin and S.P. Novikov, Ground states in a periodic field. Magnetic-Bloch functions and vector bundles, Dokl. Akad. Nauk SSSR 253 (1980), 1293-1297. = Soviet Math. Dok1. 21 (1980), 240-244.

[38] A. Andreotti and A.L. Mayer, On periodic relations for Abelian integrals on algebraic curves, Ann. Scuola Norm. Sup. Pisa (3) 21 (1967), 189-238. MR 36 \# 3792.

[39] H.M. Farkas and H.E. Rauch, Period relations of Schottky type on Riemann surfaces, Ann. of Math. (2) 92 (1970), 434-461. MR 44 \# 426.

[40] R. Garnier, Sur une classe de système différentielles abéliens déduits de la théorie des équations linéaires, Rend. Circ. Mat. Palermo 43 (1919), 155-191.

[41] S.V. Manakov, A remark on the integration of the Euler equations of the dynamics of an $n$-dimensional rigid body, Funktsional. Anal. i Prilozhen. 10:4 (1976), 93-94. MR 56 \# 13272.

= Functional Anal. Appl. 10 (1976), 328-329.

[42] B.A. Dubrovin, Completely integrable Hamiltonian systems associated with matrix operators, and Abelian varieties, Funktsional Anal. i Prilozhen. 11:4 (1977), 28-41. MR 58 \# 31219.

= Functional Anal. Appl. 11 (1977), 265-277.

[43] A.M. Perelomov, Coherent states and theta-functions, Funktsional. Anal. i Prilozhen. 6:4 (1972), 47-57. MR 57 \# 18562.

= Functional Anal. Appl. 6 (1972), 292-300.

[44] V.I. Arnold, Matematicheskie metody klassicheskoi mekhaniki, Nauka, Moscow 1974. MR 57 \# 14032.

Translation: Mathematical methods in classical mechanics, Springer-Verlag, BerlinHeidelberg-New York 1978.

[45] B.A. Dubrovin, The Kadomtsev-Petviashvili equation and relations between the periods of holomorphic differentials on a Riemann surface, Izv. Akad. Nauk SSSR Ser. Mat. 45 (1981), 1015-1028.

$=$ Math. USSR-Izv. 18 (1981).

[46] I.M. Krichever, Algebraic curves and non-linear difference equations, Uspekhi Mat. Nauk 33:4 (1978), 215-216. MR 80k:58055.

$=$ Russian Math. Survey 33:4 (1978), 255-256.

[47] D. Mumford and $P$. van Moerbeke, The spectrum of difference operators and algebraic curves, Acta Math. 143 (1979), 93-154. MR 80e:58028.

[48] A.V. Mikhailov, On the integrability of a two-dimensional generalization of the Toda chain, Zh. Eksper. Teoret. Fiz. letters 30 (1974), 443-448.

[49] I.V. Cherednik, On conditions of the reality in "finite-zone integration", Dok1. Akad. Nauk SSSR 252 (1980), 1104-1108.

[50] S. Lang, Introduction to algebraic and Abelian functions, Addison-Wesley, Reading, MA, 1972. MR 48 \# 6122.

Translation: Vvedenie v algebraicheskie i abelevy funktsii, Mir, Moscow 1976.

[51] O. Forster, Riemannsche Flächen, Springer-Verlag, Berlin-Heidelberg-New York 1977. MR 56 \# 5867.

Translation: Rimanovy poverkhnosti, Mir, Moscow 1980.

[52] F. Kötter, Die von Steklow und Liapunow entdeckten integralen Fälle der Bewegung eines Körpers in einer Flüssigkeit, Sitzungsber. Königlich Preussische Akad. d. Wiss. Berlin, 1900 , no. 6, 79-87.

[53] B.A. Dubrovin and S.P. Novikov, Periodic and conditionally periodic analogues of the multi-soliton solutions of the Korteweg-de Vries equation, Zh. Eksper. Teoret. Fiz. 67 (1974), 2131 -2143. MR 52 \# 3759.

= Soviet Physics JETP 40 (1974), 1058-1063. 
[54] B.A. Dubrovin, Periodic problems for the Korteweg-de Vries equation in the class of finite-zone potentials, Funktsional. Anal. i Prilozhen. 9:3 (1975), 41-51. = Functional Anal. Appl. 9 (1975), 215-223.

[55] P.D. Lax, Periodic solutions of Korteweg-de Vries equation, Comm. Pure Appl. Math. 28 (1975), $141-188$. MR 51 \# 6192.

[56] H.P. McKean and P. van Moerbeke, The spectrum of Hill's equation, Invent. Math. 30 (1975), 217-274. MR 53 \# 936.

[57] V.A. Marchenko, "The theory of Sturm-Liouville operators", Kiev, "Naukova Dumka", 1977 (in Russian).

[58] H.P. McKean and E. Trubowitz, Hill's operator and hyperelliptic function theory in the presence of infinitely many branch points, Comm. Pure Appl. Math. 29 (1967), 143-226. MR 55 \# 761.

[59] I.M. Krichever, An algebraic-geometric construction of the Zakharov-Shabat equations and their periodic solutions, Dokl. Akad. Nauk SSSR 227 (1976), 291 -294. MR 54 \# 1298.

= Soviet Math. Dok1. 17 (1976), 394-397.

[60] A.P. Its and V.B. Matveev, Schrödinger operators with a finite-zone spectrum and the $N$-soliton solutions of the Korteweg-de Vries equation, Teoret. Mat. Fiz. 23 (1975), 51-67. MR 57 \# 18570.

Translated by J.E. Jayne

Moscow State University

Received by the Editors 30 September 1980 